\title{
Limit theorem associated with Wishart matrices with application to hypothesis testing for common principal components
}

\author{
Koji Tsukuda* and Shun Matsuura ${ }^{\dagger}$
}

July 21,2020

\begin{abstract}
This study derives a new property of the Wishart distribution when the degree-of-freedom and the size of the matrix parameter of the distribution grow simultaneoulsy. Particularly, the asymptotic normality of the product of four independent Wishart matrices is shown under a high dimensional asymptotic regime. As an application of the result, a statistical test procedure for the common principal components hypothesis is proposed. For this problem, the proposed test statistic is asymptotically normal under the null hypothesis. In addition, the proposed test statistic diverges to positive infinity in probability under the alternative hypothesis.
\end{abstract}

keywords: asymptotic test; central limit theorem; common principal components model; high-dimension; Wishart distribution.

MSC2020 subject classifications: primary 60F05, 62F05; secondary $62 \mathrm{H} 25$.

\section{Introduction}

This paper shows the asymptotic normality of the trace of products of four independent Wishart matrices in a highdimensional setting, and proposes a statistical test procedure for the common principal components (CPC) hypothesis which is a typical null hypothesis in the context of multivariate statistical analysis.

A classical setting in multivariate analysis is that population distributions are normal and that the number of observed variables are much less than the number of individuals in a sample. In 1928, John Wishart derived the celebrated Wishart distribution as the distribution of a scatter matrix $\sum_{i=1}^{n} \boldsymbol{v}_{i} \boldsymbol{v}_{i}^{\prime}$ calculated from iid centered $p$-dimensional normal vectors $\left\{\boldsymbol{v}_{i}\right\}_{i=1}^{n}$, where' denotes the transpose. Starting from the derivation of the Wishart distribution, a lot of studies have investigated its asymptotic properties under the traditional multivariate analysis setting: $n \rightarrow \infty$ with fixed $p$. On the other hand, as observed variables have increased with the development of information technology, different settings have become possible and so multivariate statistical methods have been developed to deal with this situation. In particular, when few variables are observed, the likelihood ratio test is quite useful to test hypotheses about population covariance matrices. However, when more variables are observed than the number of the individuals in samples (so-called high-dimensional setting), the likelihood ratio test is unavailable in many cases because the scatter matrices are not full-rank. In one-sample testing problems such as "the population covariance matrix is an identity matrix", "the covariance population matrix is spherical", and "the covariance matrix is diagonal", alternative test procedures with clever usage of the trace of some functions of a scatter matrix have been proposed. Such procedures are considered to be effective in high-dimensional settings; see, e.g., Chen et al. [4], Srivastava [14], and Srivastava et al. [16].

In two-sample testing problems for covariance matrices, the hypotheses such as "two covariance population matrices are identical", "two population covariance matrices are proportional", and "two population covariance matrices have the same eigenvectors (CPC hypothesis)" have been considered. These three hypotheses testing are especially typical in two sample problems in the multivariate analysis. Indeed, they correspond Flury's hierarchical model, and the likelihood ratio test can be used for model selection [7]. As the likelihood ratio test for these hypotheses testing is unavailable in a high-dimensional setting, alternative test procedures have been proposed for the former two hypotheses (equality and proportionality); see, e.g., Li and Chen [11], Liu et al. [12], Schott [13], Srivastava and Yanagihara [15], Srivastava et al. [16], Tsukuda and Matsuura [17] and $\mathrm{Xu}$ et al. [18]. Particularly, some of them adopt test statistics based on the trace of some functions of scatter matrices. Testing the CPC hypothesis was first considered by Flury [5], and several studies including Boente et al. [2], Boik [3] and Hallin et al. [9, 10] have proposed test procedures. However, none of them have considered high-dimensional settings. Therefore, in this paper, we propose a test procedure for CPC hypothesis in a high-dimensional setting by applying our main result.

\footnotetext{
*Faculty of Mathematics, Kyushu University, 744 Motooka, Nishi-ku, Fukuoka-shi, Fukuoka 819-0395, Japan.

${ }^{\dagger}$ Faculty of Science and Technology, Keio University, 3-14-1 Hiyoshi, Kohoku-ku, Yokohama, Kanagawa 223-8522, Japan.
} 
This paper is organized as follows. In Section 2, we present the main result with the outline of its proof. An application of the limit theorem to testing CPC hypothesis is given in Section 3. Section 4 is devoted to prepare preliminary results which are used in the proof of the main result. Section 5 supplements the technically missing part in the former sections and concludes the proof of the main result.

\section{Limit theorem}

\subsection{Problem setting and assumption}

Let $n_{a}, n_{b}, n_{c}, n_{d}$ and $p$ be positive integers and $\boldsymbol{\Sigma}_{a}, \boldsymbol{\Sigma}_{b}, \boldsymbol{\Sigma}_{c}$ and $\boldsymbol{\Sigma}_{d}$ positive definite matrices. Consider four independent Wishart matrices

$$
\begin{aligned}
& \boldsymbol{T}_{a}\left(n_{a}\right) \sim W_{p}\left(n_{a}, \boldsymbol{\Sigma}_{a}\right), \boldsymbol{T}_{b}\left(n_{b}\right) \sim W_{p}\left(n_{b}, \boldsymbol{\Sigma}_{b}\right), \\
& \boldsymbol{T}_{c}\left(n_{c}\right) \sim W_{p}\left(n_{c}, \boldsymbol{\Sigma}_{c}\right), \boldsymbol{T}_{d}\left(n_{d}\right) \sim W_{p}\left(n_{d}, \boldsymbol{\Sigma}_{d}\right),
\end{aligned}
$$

where $\boldsymbol{T} \sim W_{p}(n, \boldsymbol{\Sigma})$ denotes a random $p \times p$ matrix $\boldsymbol{T}$ follows the $p$-dimensional Wishart distribution with its degreesof-freedom $n$ and its matrix parameter $\boldsymbol{\Sigma}$. We will study the asymptotic behavior of

$$
M=\frac{1}{r_{p}} \operatorname{tr}\left(\boldsymbol{T}_{a}\left(n_{a}\right) \boldsymbol{T}_{b}\left(n_{b}\right) \boldsymbol{T}_{c}\left(n_{c}\right) \boldsymbol{T}_{d}\left(n_{d}\right)\right)
$$

under the following high-dimensional asymptotic regime

$$
n_{a}, n_{b}, n_{c}, n_{d} \asymp p^{\delta}, \quad 0<\delta<1,
$$

where

$$
r_{p}=r_{p, n_{a}, n_{b}, n_{c}, n_{d}}=p^{2} \sqrt{n_{a} n_{b} n_{c} n_{d}} .
$$

To provide our limit theorem, we pose the following assumption.

Assumption 1. As $p \rightarrow \infty$ with (2.1), it holds that

$$
\begin{aligned}
& \frac{\operatorname{tr}\left(\boldsymbol{\Sigma}_{i} \boldsymbol{\Sigma}_{j}\right)}{p} \rightarrow \sigma_{i j} \in(0, \infty), \\
& \frac{\operatorname{tr}\left(\boldsymbol{\Sigma}_{i} \boldsymbol{\Sigma}_{j} \boldsymbol{\Sigma}_{k}\right)}{p} \rightarrow \sigma_{i j k} \in(-\infty, \infty), \\
& \quad \vdots \\
& \frac{\operatorname{tr}\left(\boldsymbol{\Sigma}_{i} \boldsymbol{\Sigma}_{j} \boldsymbol{\Sigma}_{k} \boldsymbol{\Sigma}_{l} \boldsymbol{\Sigma}_{i^{\prime}} \boldsymbol{\Sigma}_{j^{\prime}} \boldsymbol{\Sigma}_{k^{\prime}} \boldsymbol{\Sigma}_{l^{\prime}} \boldsymbol{\Sigma}_{i^{\prime \prime}} \boldsymbol{\Sigma}_{j^{\prime \prime}} \boldsymbol{\Sigma}_{k^{\prime \prime}} \boldsymbol{\Sigma}_{l^{\prime \prime}} \boldsymbol{\Sigma}_{i^{\prime \prime \prime}} \boldsymbol{\Sigma}_{j^{\prime \prime \prime}} \boldsymbol{\Sigma}_{k^{\prime \prime \prime}} \boldsymbol{\Sigma}_{l^{\prime \prime \prime}}\right)}{p} \\
& \rightarrow \sigma_{i j k l i^{\prime} j^{\prime} k^{\prime} l^{\prime} i^{\prime \prime} j^{\prime \prime} k^{\prime \prime} l^{\prime \prime} i^{\prime \prime \prime} j^{\prime \prime \prime} k^{\prime \prime \prime} l^{\prime \prime \prime}} \in(-\infty, \infty)
\end{aligned}
$$

for $i, j, k, l, i^{\prime}, j^{\prime}, k^{\prime}, l^{\prime}, i^{\prime \prime}, j^{\prime \prime}, k^{\prime \prime}, l^{\prime \prime}, i^{\prime \prime \prime}, j^{\prime \prime \prime}, k^{\prime \prime \prime}, l^{\prime \prime \prime}=a, b, c, d$.

Obviously, it holds that

$$
\mathrm{E}[M]=\frac{n_{a} n_{b} n_{c} n_{d}}{r_{p}} \operatorname{tr}\left(\boldsymbol{\Sigma}_{a} \boldsymbol{\Sigma}_{b} \boldsymbol{\Sigma}_{c} \boldsymbol{\Sigma}_{d}\right) .
$$

Moreover, the following proposition provides the limit of variance of $M$ under our asymptotic regime.

Proposition 2.1. Under Assumption 1, it holds that

$$
\mathrm{V}[M] \rightarrow \sigma_{a b} \sigma_{a d} \sigma_{b c} \sigma_{c d}
$$

as $p \rightarrow \infty$ with (2.1).

Proof. See Section 5.

To close this subsection, let us define four independent iid $p$-dimensional random sequences $\left\{\boldsymbol{x}_{i}\right\}_{i=1}^{n_{a}},\left\{\boldsymbol{y}_{i}\right\}_{i=1}^{n_{b}},\left\{\boldsymbol{z}_{i}\right\}_{i=1}^{n_{c}}$ and $\left\{\boldsymbol{w}_{i}\right\}_{i=1}^{n_{d}}$ which satisfy

$$
\boldsymbol{T}_{a}\left(n_{a}\right)=\sum_{i=1}^{n_{a}} \boldsymbol{x}_{i} \boldsymbol{x}_{i}^{\prime}, \boldsymbol{T}_{b}\left(n_{b}\right)=\sum_{i=1}^{n_{b}} \boldsymbol{y}_{i} \boldsymbol{y}_{i}^{\prime}, \boldsymbol{T}_{c}\left(n_{c}\right)=\sum_{i=1}^{n_{c}} \boldsymbol{z}_{i} \boldsymbol{z}_{i}^{\prime}, \boldsymbol{T}_{d}\left(n_{d}\right)=\sum_{i=1}^{n_{d}} \boldsymbol{w}_{i} \boldsymbol{w}_{i}^{\prime}
$$

where

$$
\boldsymbol{x}_{i} \sim N_{p}\left(\mathbf{0}_{p}, \boldsymbol{\Sigma}_{a}\right)\left(i=1, \ldots, n_{a}\right), \boldsymbol{y}_{i} \sim N_{p}\left(\mathbf{0}_{p}, \boldsymbol{\Sigma}_{b}\right)\left(i=1, \ldots, n_{b}\right),
$$




$$
\boldsymbol{z}_{i} \sim N_{p}\left(\mathbf{0}_{p}, \boldsymbol{\Sigma}_{c}\right)\left(i=1, \ldots, n_{c}\right), \boldsymbol{w}_{i} \sim N_{p}\left(\mathbf{0}_{p}, \boldsymbol{\Sigma}_{d}\right)\left(i=1, \ldots, n_{d}\right) .
$$

Moreover, for later discussions, let us denote

$$
\begin{aligned}
& \boldsymbol{T}_{a}(h)=\sum_{i=1}^{h} \boldsymbol{x}_{i} \boldsymbol{x}_{i}^{\prime}\left(h=1, \ldots, n_{a}\right), \boldsymbol{T}_{b}(h)=\sum_{i=1}^{h} \boldsymbol{y}_{i} \boldsymbol{y}_{i}^{\prime}\left(h=1, \ldots, n_{b}\right), \\
& \boldsymbol{T}_{c}(h)=\sum_{i=1}^{h} \boldsymbol{z}_{i} \boldsymbol{z}_{i}^{\prime}\left(h=1, \ldots, n_{c}\right), \boldsymbol{T}_{d}(h)=\sum_{i=1}^{h} \boldsymbol{w}_{i} \boldsymbol{w}_{i}^{\prime}\left(h=1, \ldots, n_{d}\right) .
\end{aligned}
$$

\subsection{Main result}

The main result of this paper is the following theorem.

Theorem 2.2. Under Assumption 1, it holds that

$$
M-\mathrm{E}[M] \Rightarrow N\left(0, \sigma_{a b} \sigma_{a d} \sigma_{b c} \sigma_{c d}\right)
$$

as $p \rightarrow \infty$ with (2.1), where $\Rightarrow$ denotes the convergence in distribution.

Proof. Define a sequence $\left\{\boldsymbol{u}_{i}\right\}_{i=1}^{n_{a}+n_{b}+n_{c}+n_{d}}$ by

$$
\begin{aligned}
\boldsymbol{u}_{i} & =\boldsymbol{x}_{i}\left(i=1, \ldots, n_{a}\right), \boldsymbol{u}_{n_{a}+i}=\boldsymbol{y}_{i}\left(i=1, \ldots, n_{b}\right), \\
\boldsymbol{u}_{n_{a}+n_{b}+i} & =\boldsymbol{z}_{i}\left(i=1, \ldots, n_{c}\right), \boldsymbol{u}_{n_{a}+n_{b}+n_{c}+i}=\boldsymbol{w}_{i}\left(i=1, \ldots, n_{d}\right) .
\end{aligned}
$$

Moreover, introduce a filtration $\left\{\mathcal{F}_{h}\right\}_{h=1}^{n_{a}+n_{b}+n_{c}+n_{d}}$ defined by $\mathcal{F}_{h}=\sigma\left(\boldsymbol{u}_{1}, \ldots, \boldsymbol{u}_{h}\right)\left(h=1, \ldots, n_{a}+n_{b}+n_{c}+n_{d}\right)$. Consider a martingale difference array $\left\{D_{h}\right\}_{h=1}^{n_{a}+\bar{n}_{b}+n_{c}+n_{d}}$ defined by

$$
D_{h}=\mathrm{E}_{h}[M]-\mathrm{E}_{h-1}[M] \quad\left(h=1, \ldots, n_{a}+n_{b}+n_{c}+n_{d}\right),
$$

where we use the notation $\mathrm{E}_{0}[\cdot]=\mathrm{E}[\cdot]$ and $\mathrm{E}_{h}[\cdot]=\mathrm{E}\left[\cdot \mid \mathcal{F}_{h}\right]\left(h=1, \ldots, n_{a}+\cdots+n_{d}\right)$ for simplicity. From the definition, it holds that

$$
\begin{aligned}
\sum_{h=1}^{n_{a}+n_{b}+n_{c}+n_{d}} D_{h} & =M-\mathrm{E}[M] \\
& =\frac{1}{r_{p}} \operatorname{tr}\left(\boldsymbol{T}_{a}\left(n_{a}\right) \boldsymbol{T}_{b}\left(n_{b}\right) \boldsymbol{T}_{c}\left(n_{c}\right) \boldsymbol{T}_{d}\left(n_{d}\right)\right)-\frac{n_{a} n_{b} n_{c} n_{d}}{r_{p}} \operatorname{tr}\left(\boldsymbol{\Sigma}_{a} \boldsymbol{\Sigma}_{b} \boldsymbol{\Sigma}_{c} \boldsymbol{\Sigma}_{d}\right) .
\end{aligned}
$$

Its quadratic variation is denoted by $\left\{\sigma_{h}^{2}\right\}_{h=1}^{n_{a}+n_{b}+n_{c}+n_{d}}$; i.e.,

$$
\sigma_{h}^{2}=\mathrm{E}_{h-1}\left[D_{h}^{2}\right] \quad\left(h=1, \ldots, n_{a}+n_{b}+n_{c}+n_{d}\right)
$$

It holds that

$$
\sigma_{h}^{2}=\mathrm{E}_{h-1}\left[\left(\mathrm{E}_{h}[M]\right)^{2}\right]-\left(\mathrm{E}_{h-1}[M]\right)^{2}\left(h=1, \ldots, n_{a}+n_{b}+n_{c}+n_{d}\right)
$$

and so

$$
\begin{aligned}
\mathrm{E}\left[\sum_{h=1}^{n_{a}+n_{b}+n_{c}+n_{d}} \sigma_{h}^{2}\right] & =\mathrm{E}\left[\sum_{h=1}^{n_{a}+n_{b}+n_{c}+n_{d}}\left\{\mathrm{E}_{h-1}\left[\left(\mathrm{E}_{h}[M]\right)^{2}\right]-\left(\mathrm{E}_{h-1}[M]\right)^{2}\right\}\right] \\
& =\sum_{h=1}^{n_{a}+n_{b}+n_{c}+n_{d}}\left\{\mathrm{E}\left[\left(\mathrm{E}_{h}[M]\right)^{2}\right]-\mathrm{E}\left[\left(\mathrm{E}_{h-1}[M]\right)^{2}\right]\right\} \\
& =\mathrm{E}\left[\left(\mathrm{E}_{n_{a}+n_{b}+n_{c}+n_{d}}[M]\right)^{2}\right]-\mathrm{E}\left[\left(\mathrm{E}_{0}[M]\right)^{2}\right] \\
& =\mathrm{E}\left[M^{2}\right]-(\mathrm{E}[M])^{2}=\mathrm{V}[M] .
\end{aligned}
$$

As we will see in Section 5, the following two lemmas hold:

Lemma 2.3. Under Assumption 1, it holds that

$$
\mathrm{V}\left[\sum_{h=1}^{n_{a}+n_{b}+n_{c}+n_{d}} \sigma_{h}^{2}\right] \rightarrow 0
$$

as $p \rightarrow \infty$ with (2.1). 
Lemma 2.4. Under Assumption 1, it holds that

$$
\sum_{h=1}^{n_{a}+n_{b}+n_{c}+n_{d}} \mathrm{E}\left[D_{h}^{4}\right] \rightarrow 0
$$

as $p \rightarrow \infty$ with (2.1).

Proposition 2.1, (2.2) and these lemmas yield that

$$
\sum_{h=1}^{n_{a}+n_{b}+n_{c}+n_{d}} \sigma_{h}^{2} \rightarrow^{p} \sigma_{a b} \sigma_{a d} \sigma_{b c} \sigma_{c d}
$$

and

$$
\frac{\sum_{h=1}^{n_{a}+n_{b}+n_{c}+n_{d}} \mathrm{E}\left[D_{h}^{4}\right]}{(\mathrm{V}[M])^{2}} \rightarrow 0 .
$$

Therefore, the conclusion follows from the martingale central limit theorem. Indeed, the Lyapunov condition follows from (2.3). This completes the proof.

\section{Testing for common principal components model}

\subsection{Problem setting}

The Common Principal Components (CPC) model is the model that the eigenvectors of the covariance matrices of (more than) two populations are identical. The CPC model was first introduced in [5], and fundamental asymptotic theory of statistical inference was established in [6]. Flury $[5,6]$ considered that the population distributions are normal. Afterwards, the CPC model was discussed in Hallin et al. [8] for other elliptical and possibly heterokurtic distributions than the normal distribution. As introduced in Section 1, tests for CPC model have been studied in Boente et al. [2], Boik [3] and Hallin et al. [9, 10], but CPC test under the high-dimensional setting has not been studied in the literature. In this section, we consider the problem by using Theorem 2.2 .

Denote spectral decompositions of two population covariance matrices $\boldsymbol{\Sigma}_{x}$ and $\boldsymbol{\Sigma}_{y}$ be $\boldsymbol{\Sigma}_{x}=\boldsymbol{U}_{x} \boldsymbol{\Lambda}_{x} \boldsymbol{U}_{x}^{\prime}$ and $\boldsymbol{\Sigma}_{y}=$ $\boldsymbol{U}_{y} \boldsymbol{\Lambda}_{y} \boldsymbol{U}_{y}^{\prime}$, respectively. The CPC model means that there exist spectral decompositions satisfying $\boldsymbol{U}_{x}=\boldsymbol{U}_{y}$. It is wellknown that this model is equivalently expressed as $\boldsymbol{\Sigma}_{x} \boldsymbol{\Sigma}_{y}=\boldsymbol{\Sigma}_{y} \boldsymbol{\Sigma}_{x}$.

Henceforth, let $p, m, n$ be positive integers, and let $\boldsymbol{\Sigma}_{x}$ and $\boldsymbol{\Sigma}_{y}$ be $p \times p$ positive-definite matrices. Suppose that we have a random sample of size $M=4 m$ from $N_{p}\left(\boldsymbol{\mu}_{x}, \boldsymbol{\Sigma}_{x}\right)$, and the sample is randomly split to four subsamples of size $m$. In the same way, suppose that we have a random sample of size $N=4 n$ from $N_{p}\left(\boldsymbol{\mu}_{y}, \boldsymbol{\Sigma}_{y}\right)$, and the sample is split to four subsamples of size $n$. Under this setting, we wish to test

$$
\begin{aligned}
& \mathcal{H}_{0}(\text { Null }) \quad: \boldsymbol{\Sigma}_{x} \boldsymbol{\Sigma}_{y}=\boldsymbol{\Sigma}_{y} \boldsymbol{\Sigma}_{x} \text {, } \\
& \mathcal{H}_{1} \text { (Alternative) : } \boldsymbol{\Sigma}_{x} \boldsymbol{\Sigma}_{y} \neq \boldsymbol{\Sigma}_{y} \boldsymbol{\Sigma}_{x} \text {. }
\end{aligned}
$$

We consider the asymptotic regime $p \rightarrow \infty$ with

$$
m, n \asymp p^{\delta}, \quad 0<\delta<1 .
$$

When the power of the test is discussed, the regime is limited to $1 / 2<\delta<1$ in order to guarantee the consistency. The following assumption is posed on the covariane matrices.

Assumption 2. As $p \rightarrow \infty$ with (3.1), it holds that

$$
\begin{aligned}
& \frac{\operatorname{tr}\left(\boldsymbol{\Sigma}_{i} \boldsymbol{\Sigma}_{j}\right)}{p} \rightarrow \sigma_{i j} \in(0, \infty), \\
& \vdots \\
& \frac{\operatorname{tr}\left(\boldsymbol{\Sigma}_{i} \boldsymbol{\Sigma}_{j} \boldsymbol{\Sigma}_{k} \boldsymbol{\Sigma}_{l} \boldsymbol{\Sigma}_{i^{\prime}}\right)}{p} \rightarrow \sigma_{i j k l i^{\prime}} \in(0, \infty), \\
& \frac{\operatorname{tr}\left(\boldsymbol{\Sigma}_{i} \boldsymbol{\Sigma}_{j} \boldsymbol{\Sigma}_{k} \boldsymbol{\Sigma}_{l} \boldsymbol{\Sigma}_{i^{\prime}} \boldsymbol{\Sigma}_{j^{\prime}}\right)}{p} \rightarrow \sigma_{i j k l i^{\prime} j^{\prime}} \in(-\infty, \infty), \\
& \quad \vdots \quad \frac{\operatorname{tr}\left(\boldsymbol{\Sigma}_{i} \boldsymbol{\Sigma}_{j} \boldsymbol{\Sigma}_{k} \boldsymbol{\Sigma}_{l} \boldsymbol{\Sigma}_{i^{\prime}} \boldsymbol{\Sigma}_{j^{\prime}} \boldsymbol{\Sigma}_{k^{\prime}} \boldsymbol{\Sigma}_{l^{\prime}} \boldsymbol{\Sigma}_{i^{\prime \prime}} \boldsymbol{\Sigma}_{j^{\prime \prime}} \boldsymbol{\Sigma}_{k^{\prime \prime}} \boldsymbol{\Sigma}_{l^{\prime \prime}} \boldsymbol{\Sigma}_{i^{\prime \prime \prime}} \boldsymbol{\Sigma}_{j^{\prime \prime \prime}} \boldsymbol{\Sigma}_{k^{\prime \prime \prime}} \boldsymbol{\Sigma}_{l^{\prime \prime \prime}}\right)}{p} \\
& \rightarrow \sigma_{i j k l i^{\prime} j^{\prime} k^{\prime} l^{\prime} i^{\prime \prime} j^{\prime \prime} k^{\prime \prime} l^{\prime \prime} i^{\prime \prime \prime} j^{\prime \prime \prime} k^{\prime \prime \prime} l^{\prime \prime \prime}} \in(-\infty, \infty)
\end{aligned}
$$

for $i, j, k, l, i^{\prime}, j^{\prime}, k^{\prime}, l^{\prime}, i^{\prime \prime}, j^{\prime \prime}, k^{\prime \prime}, l^{\prime \prime}, i^{\prime \prime \prime}, j^{\prime \prime \prime}, k^{\prime \prime \prime}, l^{\prime \prime \prime}=x, y$. 


\subsection{Test procedure}

We can equivalently transform $\mathcal{H}_{0}$ as follows:

$$
\begin{aligned}
& \mathcal{H}_{0}: \boldsymbol{\Sigma}_{x} \boldsymbol{\Sigma}_{y}=\boldsymbol{\Sigma}_{y} \boldsymbol{\Sigma}_{x} \\
& \Longleftrightarrow \mathcal{H}_{0}: \operatorname{tr}\left\{\left(\boldsymbol{\Sigma}_{x} \boldsymbol{\Sigma}_{y}-\boldsymbol{\Sigma}_{y} \boldsymbol{\Sigma}_{x}\right)\left(\boldsymbol{\Sigma}_{x} \boldsymbol{\Sigma}_{y}-\boldsymbol{\Sigma}_{y} \boldsymbol{\Sigma}_{x}\right)^{\prime}\right\}=0 \\
& \Longleftrightarrow \mathcal{H}_{0}: \operatorname{tr}\left\{\left(\boldsymbol{\Sigma}_{x} \boldsymbol{\Sigma}_{y}-\boldsymbol{\Sigma}_{y} \boldsymbol{\Sigma}_{x}\right)\left(\boldsymbol{\Sigma}_{y} \boldsymbol{\Sigma}_{x}-\boldsymbol{\Sigma}_{x} \boldsymbol{\Sigma}_{y}\right)\right\}=0 \\
& \Longleftrightarrow \mathcal{H}_{0}: \operatorname{tr}\left(\boldsymbol{\Sigma}_{x} \boldsymbol{\Sigma}_{x} \boldsymbol{\Sigma}_{y} \boldsymbol{\Sigma}_{y}\right)-\operatorname{tr}\left(\boldsymbol{\Sigma}_{x} \boldsymbol{\Sigma}_{y} \boldsymbol{\Sigma}_{x} \boldsymbol{\Sigma}_{y}\right)=0 .
\end{aligned}
$$

Hence, $\mathcal{H}_{0}$ can be equivalently transformed into $\mathcal{H}_{0}: \theta=0$, where

$$
\begin{gathered}
\theta=\theta_{p}=\sigma_{x x y y}(p)-\sigma_{x y x y}(p), \\
\sigma_{x x y y}(p)=\frac{\operatorname{tr}\left(\boldsymbol{\Sigma}_{x} \boldsymbol{\Sigma}_{x} \boldsymbol{\Sigma}_{y} \boldsymbol{\Sigma}_{y}\right)}{p}, \sigma_{x y x y}(p)=\frac{\operatorname{tr}\left(\boldsymbol{\Sigma}_{x} \boldsymbol{\Sigma}_{y} \boldsymbol{\Sigma}_{x} \boldsymbol{\Sigma}_{y}\right)}{p} .
\end{gathered}
$$

Moreover, $\mathcal{H}_{1}$ can be equivalently transformed into $\mathcal{H}_{1}: \theta>0$.

Let us denote the scatter matrices calculated from split subsamples by

$$
\boldsymbol{T}_{x 1}, \boldsymbol{T}_{x 2}, \boldsymbol{T}_{x 3}, \boldsymbol{T}_{x 4}, \boldsymbol{T}_{y 1}, \boldsymbol{T}_{y 2}, \boldsymbol{T}_{y 3}, \boldsymbol{T}_{y 4}
$$

In this case, it holds that

$$
\boldsymbol{T}_{x k} \sim W_{p}\left(m-1, \boldsymbol{\Sigma}_{x}\right)(k=1,2,3,4)
$$

and

$$
\boldsymbol{T}_{y k} \sim W_{p}\left(n-1, \boldsymbol{\Sigma}_{y}\right)(k=1,2,3,4) .
$$

Clearly,

$$
\hat{\theta}=\frac{1}{(m-1)^{2}(n-1)^{2} p}\left\{\operatorname{tr}\left(\boldsymbol{T}_{x 1} \boldsymbol{T}_{x 2} \boldsymbol{T}_{y 1} \boldsymbol{T}_{y 2}\right)-\operatorname{tr}\left(\boldsymbol{T}_{x 3} \boldsymbol{T}_{y 3} \boldsymbol{T}_{x 4} \boldsymbol{T}_{y 4}\right)\right\}
$$

is an unbiased estimator of $\theta=\sigma_{x x y y}(p)-\sigma_{x y x y}(p)$. As for the variance of $\frac{(m-1)(n-1)}{p} \hat{\theta}$, it follows from Proposition 2.1 that

$$
\begin{aligned}
\mathrm{V}[ & \left.\frac{(m-1)(n-1)}{p} \hat{\theta}\right] \\
= & \mathrm{V}\left[\frac{1}{(m-1)(n-1) p^{2}}\left\{\operatorname{tr}\left(\boldsymbol{T}_{x 1} \boldsymbol{T}_{x 2} \boldsymbol{T}_{y 1} \boldsymbol{T}_{y 2}\right)-\operatorname{tr}\left(\boldsymbol{T}_{x 3} \boldsymbol{T}_{y 3} \boldsymbol{T}_{x 4} \boldsymbol{T}_{y 4}\right)\right\}\right] \\
= & \mathrm{V}\left[\frac{1}{(m-1)(n-1) p^{2}} \operatorname{tr}\left(\boldsymbol{T}_{x 1} \boldsymbol{T}_{x 2} \boldsymbol{T}_{y 1} \boldsymbol{T}_{y 2}\right)\right] \\
& +\mathrm{V}\left[\frac{1}{(m-1)(n-1) p^{2}} \operatorname{tr}\left(\boldsymbol{T}_{x 3} \boldsymbol{T}_{y 3} \boldsymbol{T}_{x 4} \boldsymbol{T}_{y 4}\right)\right] \\
\rightarrow & \sigma_{x x} \sigma_{y y} \sigma_{x y}^{2}+\sigma_{x y}^{4} .
\end{aligned}
$$

The following proposition establishes the asymptotic behavior of $\hat{\theta}$ under our asymptotic regime.

Proposition 3.1. Under Assumption 2, it holds that

$$
\frac{(m-1)(n-1)}{p}(\hat{\theta}-\theta) \Rightarrow N\left(0, \sigma_{x x} \sigma_{y y} \sigma_{x y}^{2}+\sigma_{x y}^{4}\right)
$$

as $p \rightarrow \infty$ with (3.1).

Proof. The left-hand side equals

$$
\begin{aligned}
& \frac{1}{(m-1)(n-1) p^{2}} \operatorname{tr}\left(\boldsymbol{T}_{x 1} \boldsymbol{T}_{x 2} \boldsymbol{T}_{y 1} \boldsymbol{T}_{y 2}\right)-\frac{(m-1)(n-1)}{p} \sigma_{x x y y}(p) \\
& -\left\{\frac{1}{(m-1)(n-1) p^{2}} \operatorname{tr}\left(\boldsymbol{T}_{x 3} \boldsymbol{T}_{y 3} \boldsymbol{T}_{x 4} \boldsymbol{T}_{y 4}\right)-\frac{(m-1)(n-1)}{p} \sigma_{x y x y}(p)\right\} .
\end{aligned}
$$

It follows from Theorem 2.2 that

$$
\frac{1}{(m-1)(n-1) p^{2}} \operatorname{tr}\left(\boldsymbol{T}_{x 1} \boldsymbol{T}_{x 2} \boldsymbol{T}_{y 1} \boldsymbol{T}_{y 2}\right)-\frac{(m-1)(n-1)}{p} \sigma_{x x y y}(p) \Rightarrow N\left(0, \sigma_{x x} \sigma_{y y} \sigma_{x y}^{2}\right)
$$


and that

$$
\frac{1}{(m-1)(n-1) p^{2}} \operatorname{tr}\left(\boldsymbol{T}_{x 3} \boldsymbol{T}_{y 3} \boldsymbol{T}_{x 4} \boldsymbol{T}_{y 4}\right)-\frac{(m-1)(n-1)}{p} \sigma_{x y x y}(p) \Rightarrow N\left(0, \sigma_{x y}^{4}\right) .
$$

As the first and second terms of (3.2) are independent, the conclusion follows from the Slutsky theorem.

When the null hypothesis $\mathcal{H}_{0}: \theta=0$ is true, Proposition 3.1 implies that

$$
\frac{(m-1)(n-1)}{p} \hat{\theta} \Rightarrow N\left(0, \sigma_{x x} \sigma_{y y} \sigma_{x y}^{2}+\sigma_{x y}^{4}\right)
$$

Hence, constructing a consistent estimator of $\sigma_{x x} \sigma_{y y} \sigma_{x y}^{2}+\sigma_{x y}^{4}$ enables us to propose a test procedure. Let us denote by $\boldsymbol{T}_{x}$. and $\boldsymbol{T}_{y}$. the Scatter matrices calculated from two samples before splitting. Define

$$
\begin{aligned}
\hat{\sigma}_{x x} & =\frac{(M-1)^{2}}{p(M-2)(M+1)}\left[\operatorname{tr}\left(\boldsymbol{T}_{x} \cdot \boldsymbol{T}_{x} \cdot\right)-\frac{\left\{\operatorname{tr}\left(\boldsymbol{T}_{x}\right)\right\}^{2}}{M-1}\right], \\
\hat{\sigma}_{y y} & =\frac{(N-1)^{2}}{p(N-2)(N+1)}\left[\operatorname{tr}\left(\boldsymbol{T}_{y} \cdot \boldsymbol{T}_{y}\right)-\frac{\left\{\operatorname{tr}\left(\boldsymbol{T}_{y}\right)\right\}^{2}}{N-1}\right], \\
\hat{\sigma}_{x y} & =\frac{1}{p} \operatorname{tr}\left(\boldsymbol{T}_{x} \cdot \boldsymbol{T}_{y} .\right) .
\end{aligned}
$$

Remark 1. The estimators $\hat{\sigma}_{x x}$ and $\hat{\sigma}_{y y}$ are originally introduced by Bai and Saranadasa [1]. It is known that under Assumption 2, it holds that

$$
\hat{\sigma}_{x x} \rightarrow^{p} \sigma_{x x}, \hat{\sigma}_{y y} \rightarrow^{p} \sigma_{y y}, \hat{\sigma}_{x y} \rightarrow^{p} \sigma_{x y}
$$

as $p \rightarrow \infty$ with (3.1).

Let us propose the test statistic $T$ defined by

$$
T=\frac{(m-1)(n-1)}{p} \frac{\hat{\theta}}{\sqrt{\hat{\sigma}_{x x} \hat{\sigma}_{y y} \hat{\sigma}_{x y}^{2}+\hat{\sigma}_{x y}^{4}}},
$$

and we propose the following test procedure (approximate significance level is $\alpha$ ):

- If $T>\Phi^{-1}(1-\alpha)$ then reject $\mathcal{H}_{0}$;

where $\Phi^{-1}(\cdot)$ is the quantile function of the standard normal distribution. This test procedure is justified by the following corollaries. Particularly, Corollary 3.3 guarantees the consistency of our test procedure when $1 / 2<\delta<1$.

Corollary 3.2. Under Assumption 2, when $\mathcal{H}_{0}$ is true, $\mathrm{P}\left(T>\Phi^{-1}(1-\alpha)\right) \rightarrow \alpha$ as $p \rightarrow \infty$ with (3.1).

Proof. When $\mathcal{H}_{0}$ is true, Proposition 3.1 and (3.3) conjunction with the Slutsky theorem yield that $T \Rightarrow N(0,1)$ as $p \rightarrow \infty$ with (3.1). This completes the proof.

Corollary 3.3. Under Assumption 2, when $\mathcal{H}_{1}$ is true, if $\sigma_{x x y y}-\sigma_{x y x y}>0$ then $\mathrm{P}(T>C) \rightarrow 1$ for any positive constant $C$ as $p \rightarrow \infty$ with $m, n \asymp p^{\delta}, 1 / 2<\delta<1$.

Proof. It holds that

$$
\begin{aligned}
T= & \frac{(m-1)(n-1)}{p} \frac{\hat{\theta}-\theta}{\sqrt{\hat{\sigma}_{x x} \hat{\sigma}_{y y} \hat{\sigma}_{x y}^{2}+\hat{\sigma}_{x y}^{4}}}+\frac{(m-1)(n-1)}{p} \frac{\sigma_{x x y y}(p)-\sigma_{x y x y}(p)}{\sqrt{\hat{\sigma}_{x x} \hat{\sigma}_{y y} \hat{\sigma}_{x y}^{2}+\hat{\sigma}_{x y}^{4}}} \\
\geq & \frac{(m-1)(n-1)}{p} \frac{\hat{\theta}-\theta}{\sqrt{\hat{\sigma}_{x x} \hat{\sigma}_{y y} \hat{\sigma}_{x y}^{2}+\hat{\sigma}_{x y}^{4}}} \\
& +\frac{(m-1)(n-1)}{p} \frac{\inf _{q \geq p}\left\{\sigma_{x x y y}(q)-\sigma_{x y x y}(q)\right\}}{\sqrt{\hat{\sigma}_{x x} \hat{\sigma}_{y y} \hat{\sigma}_{x y}^{2}+\hat{\sigma}_{x y}^{4}}}
\end{aligned}
$$

The first term of the right-hand side is $O_{P}(1)$ by using Proposition 3.1, (3.3) and the Slutsky theorem. The second term tends to positive infinity because $(m-1)(n-1) / p \rightarrow \infty \operatorname{and} \inf _{q \geq p}\left\{\sigma_{x x y y}(q)-\sigma_{x y x y}(q)\right\} \rightarrow \sigma_{x x y y}-\sigma_{x y x y}>0$ as $p \rightarrow \infty$. This completes the proof. 
Remark 2. There is another natural unbiased estimator of $\theta$ other than $\hat{\theta}$. For instance,

$$
\begin{aligned}
& \frac{1}{(M-2)(M+1)(N-2)(N+1)}\left[\operatorname{tr}\left(\boldsymbol{T}_{x} \cdot \boldsymbol{T}_{x} \cdot \boldsymbol{T}_{y} \cdot \boldsymbol{T}_{y} \cdot\right)-\frac{1}{N-1} \operatorname{tr}\left(\boldsymbol{T}_{x} \cdot \boldsymbol{T}_{x} \cdot \boldsymbol{T}_{y} \cdot\right) \operatorname{tr}\left(\boldsymbol{T}_{y}\right)\right. \\
& -\frac{1}{M-1} \operatorname{tr}\left(\boldsymbol{T}_{x} \cdot \boldsymbol{T}_{y} \cdot \boldsymbol{T}_{y}\right) \operatorname{tr}\left(\boldsymbol{T}_{x}\right)+\frac{1}{(M-1)(N-1)} \operatorname{tr}\left(\boldsymbol{T}_{x} \cdot \boldsymbol{T}_{y} \cdot\right) \operatorname{tr}\left(\boldsymbol{T}_{x} \cdot\right) \operatorname{tr}\left(\boldsymbol{T}_{y} \cdot\right) \\
& \left.-\frac{M N-M-N+3}{(M-1)(N-1)} \operatorname{tr}\left(\boldsymbol{T}_{x} \cdot \boldsymbol{T}_{y} \cdot \boldsymbol{T}_{x} \cdot \boldsymbol{T}_{y}\right)+\frac{M+N-1}{(M-1)(N-1)} \operatorname{tr}\left(\boldsymbol{T}_{x} \cdot \boldsymbol{T}_{y} \cdot\right) \operatorname{tr}\left(\boldsymbol{T}_{x} \cdot \boldsymbol{T}_{y} \cdot\right)\right]
\end{aligned}
$$

is an unbiased estimator of $\theta$. Deriving the asymptotic behavior of this quantity is a possible future direction.

\section{Preliminary results}

\subsection{Results for quadratic form of standard normal vectors}

In this subsection, we provide some properties concerning quadratic form of standard normal vectors.

Lemma 4.1. If $\boldsymbol{x}=\left(x_{1}, \ldots, x_{p}\right) \sim N_{p}\left(\mathbf{0}_{p}, \boldsymbol{I}_{p}\right)$ then

$$
\begin{aligned}
& \mathrm{E}\left[\left(\boldsymbol{x}^{\prime} \boldsymbol{A} \boldsymbol{x}\right)^{2}\right]=2 \operatorname{tr}\left(\boldsymbol{A}^{2}\right)+\{\operatorname{tr}(\boldsymbol{A})\}^{2}, \\
& \mathrm{E}\left[\left(\boldsymbol{x}^{\prime} \boldsymbol{A} \boldsymbol{x}\right)^{3}\right]=8 \operatorname{tr}\left(\boldsymbol{A}^{3}\right)+6 \operatorname{tr}\left(\boldsymbol{A}^{2}\right) \operatorname{tr}(\boldsymbol{A})+(\operatorname{tr}(\boldsymbol{A}))^{3}, \\
& \mathrm{E}\left[\left(\boldsymbol{x}^{\prime} \boldsymbol{A} \boldsymbol{x}\right)^{4}\right] \\
& =48 \operatorname{tr}\left(\boldsymbol{A}^{4}\right)+32 \operatorname{tr}\left(\boldsymbol{A}^{3}\right) \operatorname{tr}(\boldsymbol{A})+12\left(\operatorname{tr}\left(\boldsymbol{A}^{2}\right)\right)^{2}+12 \operatorname{tr}\left(\boldsymbol{A}^{2}\right)(\operatorname{tr}(\boldsymbol{A}))^{2}+(\operatorname{tr}(\boldsymbol{A}))^{4}
\end{aligned}
$$

for any $p \times p$ symmetric matrix $\boldsymbol{A}$.

Proof. By using the spectral decomposition, $\boldsymbol{A}$ is denoted by $\boldsymbol{U} \boldsymbol{\Lambda} \boldsymbol{U}^{\prime}$, where $\boldsymbol{U}$ is an orthogonal matrix and $\boldsymbol{\Lambda}=$ $\operatorname{diag}\left(\lambda_{1}, \ldots, \lambda_{p}\right)$. It holds that

$$
\begin{gathered}
\mathrm{E}\left[\left(\boldsymbol{x}^{\prime} \boldsymbol{A} \boldsymbol{x}\right)^{2}\right]=\mathrm{E}\left[\left(\boldsymbol{x}^{\prime} \boldsymbol{\Lambda} \boldsymbol{x}\right)^{2}\right]=\mathrm{E}\left[\left(\sum_{i=1}^{p} \lambda_{i} x_{i}^{2}\right)^{2}\right]=\mathrm{E}\left[\sum_{i=1}^{p} \lambda_{i}^{2} x_{i}^{4}+\sum_{i=1}^{p} \sum_{j \neq i} \lambda_{i} x_{i}^{2} \lambda_{j} x_{j}^{2}\right] \\
=3 \sum_{i=1}^{p} \lambda_{i}^{2}+\sum_{i=1}^{p} \sum_{j \neq i} \lambda_{i} \lambda_{j}=2 \sum_{i=1}^{p} \lambda_{i}^{2}+\left(\sum_{i=1}^{p} \lambda_{i}\right)^{2}=2 \operatorname{tr}\left(\boldsymbol{A}^{2}\right)+(\operatorname{tr}(\boldsymbol{A}))^{2} .
\end{gathered}
$$

Moreover, it holds that

$$
\begin{aligned}
\mathrm{E} & {\left[\left(\boldsymbol{x}^{\prime} \boldsymbol{A} \boldsymbol{x}\right)^{3}\right]=\mathrm{E}\left[\left(\boldsymbol{x}^{\prime} \boldsymbol{\Lambda} \boldsymbol{x}\right)^{3}\right]=\mathrm{E}\left[\left(\sum_{i=1}^{p} \lambda_{i} x_{i}^{2}\right)^{3}\right] } \\
& =\mathrm{E}\left[\sum_{i=1}^{p} \lambda_{i}^{3} x_{i}^{6}+3 \sum_{i=1}^{p} \sum_{j \neq i} \lambda_{i}^{2} x_{i}^{4} \lambda_{j} x_{j}^{2}+\sum_{i=1}^{p} \sum_{j \neq i} \sum_{k \neq i, j} \lambda_{i} x_{i}^{2} \lambda_{j} x_{j}^{2} \lambda_{k} x_{k}^{2}\right] \\
& =15 \sum_{i=1}^{p} \lambda_{i}^{3}+9 \sum_{i=1}^{p} \sum_{j \neq i} \lambda_{i}^{2} \lambda_{j}+\sum_{i=1}^{p} \sum_{j \neq i} \sum_{k \neq i, j} \lambda_{i} \lambda_{j} \lambda_{k}=14 \sum_{i=1}^{p} \lambda_{i}^{3}+6 \sum_{i=1}^{p} \sum_{j \neq i} \lambda_{i}^{2} \lambda_{j}+\left(\sum_{i=1}^{p} \lambda_{i}\right)^{3} \\
& =8 \sum_{i=1}^{p} \lambda_{i}^{3}+6\left(\sum_{i=1}^{p} \lambda_{i}^{2}\right)\left(\sum_{i=1}^{p} \lambda_{i}\right)+\left(\sum_{i=1}^{p} \lambda_{i}\right)^{3}=8 \operatorname{tr}\left(\boldsymbol{A}^{3}\right)+6 \operatorname{tr}\left(\boldsymbol{A}^{2}\right) \operatorname{tr}(\boldsymbol{A})+(\operatorname{tr}(\boldsymbol{A}))^{3} .
\end{aligned}
$$

Furthermore, it holds that

$$
\begin{aligned}
& \mathrm{E}\left[\left(\boldsymbol{x}^{\prime} \boldsymbol{A} \boldsymbol{x}\right)^{4}\right]=\mathrm{E}\left[\left(\boldsymbol{x}^{\prime} \boldsymbol{\Lambda} \boldsymbol{x}\right)^{4}\right]=\mathrm{E}\left[\left(\sum_{i=1}^{p} \lambda_{i} x_{i}^{2}\right)^{4}\right] \\
& =\mathrm{E}\left[\sum_{i=1}^{p} \lambda_{i}^{4} x_{i}^{8}+4 \sum_{i=1}^{p} \sum_{j \neq i} \lambda_{i}^{3} x_{i}^{6} \lambda_{j} x_{j}^{2}+3 \sum_{i=1}^{p} \sum_{j \neq i} \lambda_{i}^{2} x_{i}^{4} \lambda_{j}^{2} x_{j}^{4}+6 \sum_{i=1}^{p} \sum_{j \neq i} \sum_{k \neq i, j} \lambda_{i}^{2} x_{i}^{4} \lambda_{j} x_{j}^{2} \lambda_{k} x_{k}^{2}\right.
\end{aligned}
$$




$$
\begin{aligned}
& \left.+\sum_{i=1}^{p} \sum_{j \neq i} \sum_{k \neq i, j} \sum_{l \neq i, j, k} \lambda_{i} x_{i}^{2} \lambda_{j} x_{j}^{2} \lambda_{k} x_{k}^{2} \lambda_{l} x_{l}^{2}\right] \\
= & 105 \sum_{i=1}^{p} \lambda_{i}^{4}+60 \sum_{i=1}^{p} \sum_{j \neq i} \lambda_{i}^{3} \lambda_{j}+27 \sum_{i=1}^{p} \sum_{j \neq i} \lambda_{i}^{2} \lambda_{j}^{2}+18 \sum_{i=1}^{p} \sum_{j \neq i} \sum_{k \neq i, j} \lambda_{i}^{2} \lambda_{j} \lambda_{k}+\sum_{i=1}^{p} \sum_{j \neq i} \sum_{k \neq i, j} \sum_{l \neq i, j, k} \lambda_{i} \lambda_{j} \lambda_{k} \lambda_{l} \\
= & 104 \sum_{i=1}^{p} \lambda_{i}^{4}+56 \sum_{i=1}^{p} \sum_{j \neq i} \lambda_{i}^{3} \lambda_{j}+24 \sum_{i=1}^{p} \sum_{j \neq i} \lambda_{i}^{2} \lambda_{j}^{2}+12 \sum_{i=1}^{p} \sum_{j \neq i} \sum_{k \neq i, j} \lambda_{i}^{2} \lambda_{j} \lambda_{k}+\left(\sum_{i=1}^{p} \lambda_{i}\right)^{4} \\
= & 92 \sum_{i=1}^{p} \lambda_{i}^{4}+32 \sum_{i=1}^{p} \sum_{j \neq i} \lambda_{i}^{3} \lambda_{j}+12 \sum_{i=1}^{p} \sum_{j \neq i} \lambda_{i}^{2} \lambda_{j}^{2}+12\left(\sum_{i=1}^{p} \lambda_{i}^{2}\right)\left(\sum_{i=1}^{p} \lambda_{i}\right)^{2}+\left(\sum_{i=1}^{p} \lambda_{i}\right)^{4} \\
= & 80 \sum_{i=1}^{p} \lambda_{i}^{4}+32 \sum_{i=1}^{p} \sum_{j \neq i} \lambda_{i}^{3} \lambda_{j}+12\left(\sum_{i=1}^{p} \lambda_{i}^{2}\right)^{2}+12\left(\sum_{i=1}^{p} \lambda_{i}^{2}\right)\left(\sum_{i=1}^{p} \lambda_{i}\right)^{2}+\left(\sum_{i=1}^{p} \lambda_{i}\right)^{4} \\
= & 48 \sum_{i=1}^{p} \lambda_{i}^{4}+32\left(\sum_{i=1}^{p} \lambda_{i}^{3}\right)\left(\sum_{i=1}^{p} \lambda_{i}\right)+12\left(\sum_{i=1}^{p} \lambda_{i}^{2}\right)^{2}+12\left(\sum_{i=1}^{p} \lambda_{i}^{2}\right)\left(\sum_{i=1}^{p} \lambda_{i}\right)^{2}+\left(\sum_{i=1}^{p} \lambda_{i}\right)^{4} \\
= & 48 \operatorname{tr}\left(\boldsymbol{\Lambda}^{4}\right)+32 \operatorname{tr}\left(\boldsymbol{\Lambda}^{3}\right) \operatorname{tr}(\boldsymbol{\Lambda})+12\left(\operatorname{tr}\left(\boldsymbol{\Lambda}^{2}\right)\right)^{2}+12 \operatorname{tr}\left(\boldsymbol{\Lambda}^{2}\right)(\operatorname{tr}(\boldsymbol{\Lambda}))^{2}+(\operatorname{tr}(\boldsymbol{\Lambda}))^{4} \\
= & 48 \operatorname{tr}\left(\boldsymbol{A}^{4}\right)+32 \operatorname{tr}\left(\boldsymbol{A}^{3}\right) \operatorname{tr}(\boldsymbol{A})+12\left(\operatorname{tr}\left(\boldsymbol{A}^{2}\right)\right)^{2}+12 \operatorname{tr}\left(\boldsymbol{A}^{2}\right)(\operatorname{tr}(\boldsymbol{A}))^{2}+(\operatorname{tr}(\boldsymbol{A}))^{4} .
\end{aligned}
$$

This completes the proof.

By using Lemma 4.1, we can show the following assertions which will be used in Section 5 .

Lemma 4.2. If $\boldsymbol{x} \sim N_{p}\left(\mathbf{0}_{p}, \boldsymbol{I}_{p}\right)$ then

$$
\mathrm{E}\left[\left\{\boldsymbol{x}^{\prime} \boldsymbol{A} \boldsymbol{x}-\operatorname{tr}(\boldsymbol{A})\right\}^{2}\right]=\operatorname{tr}(\boldsymbol{A} \boldsymbol{A})+\operatorname{tr}\left(\boldsymbol{A} \boldsymbol{A}^{\prime}\right)
$$

for any $p \times p$ matrix $\boldsymbol{A}$.

Proof. First, for symmetric $\boldsymbol{A}$, it follows from Lemma 4.1 that

$$
\mathrm{E}\left[\left\{\boldsymbol{x}^{\prime} \boldsymbol{A} \boldsymbol{x}-\operatorname{tr}(\boldsymbol{A})\right\}^{2}\right]=\mathrm{E}\left[\left(\boldsymbol{x}^{\prime} \boldsymbol{A} \boldsymbol{x}\right)^{2}-2 \boldsymbol{x}^{\prime} \boldsymbol{A} \boldsymbol{x} \operatorname{tr}(\boldsymbol{A})+\{\operatorname{tr}(\boldsymbol{A})\}^{2}\right]=2 \operatorname{tr}\left(\boldsymbol{A}^{2}\right)+\{\operatorname{tr}(\boldsymbol{A})\}^{2}-\{\operatorname{tr}(\boldsymbol{A})\}^{2}=2 \operatorname{tr}\left(\boldsymbol{A}^{2}\right) .
$$

Hence, for possibly asymmetric $\boldsymbol{A}$, it holds that

$$
\begin{aligned}
\mathrm{E} & {\left[\left\{\boldsymbol{x}^{\prime} \boldsymbol{A} \boldsymbol{x}-\operatorname{tr}(\boldsymbol{A})\right\}^{2}\right] } \\
& =\mathrm{E}\left[\left\{\boldsymbol{x}^{\prime}\left(\frac{\boldsymbol{A}+\boldsymbol{A}^{\prime}}{2}\right) \boldsymbol{x}-\operatorname{tr}\left(\frac{\boldsymbol{A}+\boldsymbol{A}^{\prime}}{2}\right)\right\}^{2}\right]=2 \operatorname{tr}\left(\left(\frac{\boldsymbol{A}+\boldsymbol{A}^{\prime}}{2}\right)^{2}\right)=\frac{1}{2} \operatorname{tr}\left(\left(\boldsymbol{A}+\boldsymbol{A}^{\prime}\right)^{2}\right) \\
& =\frac{1}{2}\left\{\operatorname{tr}(\boldsymbol{A} \boldsymbol{A})+\operatorname{tr}\left(\boldsymbol{A} \boldsymbol{A}^{\prime}\right)+\operatorname{tr}\left(\boldsymbol{A}^{\prime} \boldsymbol{A}\right)+\operatorname{tr}\left(\boldsymbol{A}^{\prime} \boldsymbol{A}^{\prime}\right)\right\}=\operatorname{tr}(\boldsymbol{A} \boldsymbol{A})+\operatorname{tr}\left(\boldsymbol{A} \boldsymbol{A}^{\prime}\right) .
\end{aligned}
$$

This completes the proof.

Lemma 4.3. If $\boldsymbol{x} \sim N_{p}\left(\mathbf{0}_{p}, \boldsymbol{I}_{p}\right)$ then

$$
\mathrm{E}\left[\left(\boldsymbol{x}^{\prime} \boldsymbol{A} \boldsymbol{x}-\operatorname{tr}(\boldsymbol{A})\right)^{4}\right]=3 \operatorname{tr}\left(\left(\boldsymbol{A}+\boldsymbol{A}^{\prime}\right)^{4}\right)+\frac{3}{4}\left\{\operatorname{tr}\left(\left(\boldsymbol{A}+\boldsymbol{A}^{\prime}\right)^{2}\right)\right\}^{2}
$$

for any $p \times p$ matrix $\boldsymbol{A}$.

Proof. First, for symmetric $\boldsymbol{A}$, it follows from Lemma 4.1 that

$$
\begin{aligned}
\mathrm{E}[ & \left.\left(\boldsymbol{x}^{\prime} \boldsymbol{A} \boldsymbol{x}-\operatorname{tr}(\boldsymbol{A})\right)^{4}\right] \\
= & \mathrm{E}\left[\left(\boldsymbol{x}^{\prime} \boldsymbol{A} \boldsymbol{x}\right)^{4}-4\left(\boldsymbol{x}^{\prime} \boldsymbol{A} \boldsymbol{x}\right)^{3} \operatorname{tr}(\boldsymbol{A})+6\left(\boldsymbol{x}^{\prime} \boldsymbol{A} \boldsymbol{x}\right)^{2}(\operatorname{tr}(\boldsymbol{A}))^{2}-4 \boldsymbol{x}^{\prime} \boldsymbol{A} \boldsymbol{x}(\operatorname{tr}(\boldsymbol{A}))^{3}+(\operatorname{tr}(\boldsymbol{A}))^{4}\right] \\
= & \left\{48 \operatorname{tr}\left(\boldsymbol{A}^{4}\right)+32 \operatorname{tr}\left(\boldsymbol{A}^{3}\right) \operatorname{tr}(\boldsymbol{A})+12\left(\operatorname{tr}\left(\boldsymbol{A}^{2}\right)\right)^{2}+12 \operatorname{tr}\left(\boldsymbol{A}^{2}\right)(\operatorname{tr}(\boldsymbol{A}))^{2}+(\operatorname{tr}(\boldsymbol{A}))^{4}\right\} \\
& -4\left\{8 \operatorname{tr}\left(\boldsymbol{A}^{3}\right)+6 \operatorname{tr}\left(\boldsymbol{A}^{2}\right) \operatorname{tr}(\boldsymbol{A})+(\operatorname{tr}(\boldsymbol{A}))^{3}\right\} \operatorname{tr}(\boldsymbol{A})+6\left\{2 \operatorname{tr}\left(\boldsymbol{A}^{2}\right)+(\operatorname{tr}(\boldsymbol{A}))^{2}\right\}(\operatorname{tr}(\boldsymbol{A}))^{2}
\end{aligned}
$$




$$
\begin{aligned}
& -4 \operatorname{tr}(\boldsymbol{A})(\operatorname{tr}(\boldsymbol{A}))^{3}+(\operatorname{tr}(\boldsymbol{A}))^{4} \\
= & \left\{48 \operatorname{tr}\left(\boldsymbol{A}^{4}\right)+32 \operatorname{tr}\left(\boldsymbol{A}^{3}\right) \operatorname{tr}(\boldsymbol{A})+12\left(\operatorname{tr}\left(\boldsymbol{A}^{2}\right)\right)^{2}+12 \operatorname{tr}\left(\boldsymbol{A}^{2}\right)(\operatorname{tr}(\boldsymbol{A}))^{2}+(\operatorname{tr}(\boldsymbol{A}))^{4}\right\} \\
& -\left\{32 \operatorname{tr}\left(\boldsymbol{A}^{3}\right)+24 \operatorname{tr}\left(\boldsymbol{A}^{2}\right) \operatorname{tr}(\boldsymbol{A})+4(\operatorname{tr}(\boldsymbol{A}))^{3}\right\} \operatorname{tr}(\boldsymbol{A})+\left\{12 \operatorname{tr}\left(\boldsymbol{A}^{2}\right)+6(\operatorname{tr}(\boldsymbol{A}))^{2}\right\}(\operatorname{tr}(\boldsymbol{A}))^{2} \\
& -4(\operatorname{tr}(\boldsymbol{A}))^{4}+(\operatorname{tr}(\boldsymbol{A}))^{4} \\
= & 48 \operatorname{tr}\left(\boldsymbol{A}^{4}\right)+12\left(\operatorname{tr}\left(\boldsymbol{A}^{2}\right)\right)^{2} .
\end{aligned}
$$

Hence, for possibly asymmetric $\boldsymbol{A}$, it holds that

$$
\begin{aligned}
\mathrm{E}\left[\left(\boldsymbol{x}^{\prime} \boldsymbol{A} \boldsymbol{x}-\operatorname{tr}(\boldsymbol{A})\right)^{4}\right] & =\mathrm{E}\left[\left(\boldsymbol{x}^{\prime}\left(\frac{\boldsymbol{A}+\boldsymbol{A}^{\prime}}{2}\right) \boldsymbol{x}-\operatorname{tr}\left(\frac{\boldsymbol{A}+\boldsymbol{A}^{\prime}}{2}\right)\right)^{4}\right] \\
& =\frac{1}{16} \mathrm{E}\left[\left(\boldsymbol{x}^{\prime}\left(\boldsymbol{A}+\boldsymbol{A}^{\prime}\right) \boldsymbol{x}-\operatorname{tr}\left(\boldsymbol{A}+\boldsymbol{A}^{\prime}\right)\right)^{4}\right] \\
& =\frac{1}{16}\left\{48 \operatorname{tr}\left(\left(\boldsymbol{A}+\boldsymbol{A}^{\prime}\right)^{4}\right)+12\left(\operatorname{tr}\left(\left(\boldsymbol{A}+\boldsymbol{A}^{\prime}\right)^{2}\right)\right)^{2}\right\} \\
& =3 \operatorname{tr}\left(\left(\boldsymbol{A}+\boldsymbol{A}^{\prime}\right)^{4}\right)+\frac{3}{4}\left\{\operatorname{tr}\left(\left(\boldsymbol{A}+\boldsymbol{A}^{\prime}\right)^{2}\right)\right\}^{2} .
\end{aligned}
$$

This completes the proof.

\subsection{Results for Wishart matrices}

In this subsection, we provide some asymptotic properties concerning Wishart matrices under a high-dimensional asymptotic regime. Throughout this section, let $a, b$, and $p$ be positive integers, and $\boldsymbol{\Sigma}, \boldsymbol{\Sigma}_{a}$, and $\boldsymbol{\Sigma}_{b}$ be $p \times p$ positive definite matrices. Before presenting results, recall that if $\boldsymbol{z} \sim N_{p}(\mathbf{0}, \boldsymbol{\Sigma})$ then

$$
\mathrm{E}\left[\left(\boldsymbol{z}^{\prime} \boldsymbol{A} \boldsymbol{z}\right) \boldsymbol{z} \boldsymbol{z}^{\prime}\right]=\mathrm{E}\left[\boldsymbol{z} \boldsymbol{z}^{\prime} \boldsymbol{A} \boldsymbol{z} \boldsymbol{z}^{\prime}\right]=\boldsymbol{\Sigma} \boldsymbol{A} \boldsymbol{\Sigma}+\boldsymbol{\Sigma} \boldsymbol{A}^{\prime} \boldsymbol{\Sigma}+\operatorname{tr}(\boldsymbol{\Sigma} \boldsymbol{A}) \boldsymbol{\Sigma}
$$

for any $p \times p$ matrix $\boldsymbol{A}$.

First we provide asymptotic evaluations for moments of a Wishart matrix.

Proposition 4.4. If $\boldsymbol{T} \sim W_{p}(a, \boldsymbol{\Sigma})$ then

$$
\mathrm{E}[\operatorname{tr}(\boldsymbol{T} \boldsymbol{A} \boldsymbol{T} \boldsymbol{B})]=O\left(a p^{2}\right), \quad \mathrm{E}[\operatorname{tr}(\boldsymbol{T} \boldsymbol{A}) \operatorname{tr}(\boldsymbol{T} \boldsymbol{B})]=O\left(a^{2} p^{2}\right)
$$

as $p \rightarrow \infty$ with

$$
a \asymp p^{\delta}, \quad 0<\delta<1 .
$$

for $p \times p$ matrices $\boldsymbol{A}$ and $\boldsymbol{B}$ which satisfy

$$
\operatorname{tr}(\boldsymbol{\Sigma} \boldsymbol{A} \boldsymbol{\Sigma} \boldsymbol{B})=O(p), \operatorname{tr}\left(\boldsymbol{\Sigma} \boldsymbol{A}^{\prime} \boldsymbol{\Sigma} \boldsymbol{B}\right)=O(p), \operatorname{tr}(\boldsymbol{\Sigma} \boldsymbol{A})=O(p), \operatorname{tr}(\boldsymbol{\Sigma} \boldsymbol{B})=O(p) .
$$

Proof Let $\left\{\boldsymbol{z}_{i}\right\}_{i=1}^{a}$ be an iid sequence satisfying $\boldsymbol{z}_{1}, \ldots, \boldsymbol{z}_{a} \sim N_{p}(\mathbf{0}, \boldsymbol{\Sigma})$. Note that

$$
\boldsymbol{T} \stackrel{d}{=} \sum_{i=1}^{a} \boldsymbol{z}_{i} \boldsymbol{z}_{i}^{\prime}
$$

where $\stackrel{d}{=}$ means that the distributions of left-hand and right-hand sides are the same. It follows from

$$
\begin{aligned}
\mathrm{E} & {[\boldsymbol{T} \boldsymbol{A} \boldsymbol{T}]=\mathrm{E}\left[\left(\sum_{i=1}^{a} \boldsymbol{z}_{i} \boldsymbol{z}_{i}^{\prime}\right) \boldsymbol{A}\left(\sum_{i=1}^{a} \boldsymbol{z}_{i} \boldsymbol{z}_{i}^{\prime}\right)\right] } \\
& =\sum_{i=1}^{a} \mathrm{E}\left[\boldsymbol{z}_{i} \boldsymbol{z}_{i}^{\prime} \boldsymbol{A} \boldsymbol{z}_{i} \boldsymbol{z}_{i}^{\prime}\right]+\sum_{i=1}^{a} \sum_{j \neq i} \mathrm{E}\left[\boldsymbol{z}_{i} \boldsymbol{z}_{i}^{\prime} \boldsymbol{A} \boldsymbol{z}_{j} \boldsymbol{z}_{j}^{\prime}\right] \\
& =\sum_{i=1}^{a}\left\{\boldsymbol{\Sigma} \boldsymbol{A} \boldsymbol{\Sigma}+\boldsymbol{\Sigma} \boldsymbol{A}^{\prime} \boldsymbol{\Sigma}+\operatorname{tr}(\boldsymbol{\Sigma} \boldsymbol{A}) \boldsymbol{\Sigma}\right\}+\sum_{i=1}^{a} \sum_{j \neq i} \boldsymbol{\Sigma} \boldsymbol{A} \boldsymbol{\Sigma} \\
& =a^{2} \boldsymbol{\Sigma} \boldsymbol{A} \boldsymbol{\Sigma}+a \boldsymbol{\Sigma} \boldsymbol{A}^{\prime} \boldsymbol{\Sigma}+a \operatorname{tr}(\boldsymbol{\Sigma} \boldsymbol{A}) \boldsymbol{\Sigma} .
\end{aligned}
$$


that

$$
\mathrm{E}[\operatorname{tr}(\boldsymbol{T} \boldsymbol{A T \boldsymbol { B }})]=O\left(a^{2} p\right)+O\left(a p^{2}\right)=O\left(a p^{2}\right) .
$$

Moreover, it follows from

$$
\begin{aligned}
\mathrm{E} & {[\operatorname{tr}(\boldsymbol{T} \boldsymbol{A}) \boldsymbol{T}]=\mathrm{E}\left[\operatorname{tr}\left(\sum_{i=1}^{a} \boldsymbol{z}_{i} \boldsymbol{z}_{i}^{\prime} \boldsymbol{A}\right)\left(\sum_{i=1}^{a} \boldsymbol{z}_{i} \boldsymbol{z}_{i}^{\prime}\right)\right] } \\
& =\mathrm{E}\left[\left(\sum_{i=1}^{a} \boldsymbol{z}_{i}^{\prime} \boldsymbol{A} \boldsymbol{z}_{i}\right)\left(\sum_{i=1}^{a} \boldsymbol{z}_{i} \boldsymbol{z}_{i}^{\prime}\right)\right] \\
& =\sum_{i=1}^{a} \mathrm{E}\left[\left(\boldsymbol{z}_{i}^{\prime} \boldsymbol{A} \boldsymbol{z}_{i}\right)\left(\boldsymbol{z}_{i} \boldsymbol{z}_{i}^{\prime}\right)\right]+\sum_{i=1}^{a} \sum_{j \neq i} \mathrm{E}\left[\left(\boldsymbol{z}_{i}^{\prime} \boldsymbol{A} \boldsymbol{z}_{i}\right)\left(\boldsymbol{z}_{j} \boldsymbol{z}_{j}^{\prime}\right)\right] \\
& =\sum_{i=1}^{a}\left\{\boldsymbol{\Sigma} \boldsymbol{A} \boldsymbol{\Sigma}+\boldsymbol{\Sigma} \boldsymbol{A}^{\prime} \boldsymbol{\Sigma}+\operatorname{tr}(\boldsymbol{\Sigma} \boldsymbol{A}) \boldsymbol{\Sigma}\right\}+\sum_{i=1}^{a} \sum_{j \neq i} \operatorname{tr}(\boldsymbol{\Sigma} \boldsymbol{A}) \boldsymbol{\Sigma} \\
& =a \boldsymbol{\Sigma} \boldsymbol{A} \boldsymbol{\Sigma}+a \boldsymbol{\Sigma} \boldsymbol{A}^{\prime} \boldsymbol{\Sigma}+a^{2} \operatorname{tr}(\boldsymbol{\Sigma} \boldsymbol{A}) \boldsymbol{\Sigma}
\end{aligned}
$$

that

$$
\mathrm{E}[\operatorname{tr}(\boldsymbol{T} \boldsymbol{A}) \operatorname{tr}(\boldsymbol{T} \boldsymbol{B})]=O\left(a^{2} p^{2}\right) .
$$

This completes the proof.

Proposition 4.5. If $\boldsymbol{T} \sim W_{p}(a, \boldsymbol{\Sigma})$ then

$$
\begin{aligned}
& \mathrm{E}[\operatorname{tr}(\boldsymbol{T} \boldsymbol{A T} \boldsymbol{B} \boldsymbol{T C})]=O\left(a p^{3}\right), \\
& \mathrm{E}[\operatorname{tr}(\boldsymbol{T} \boldsymbol{A} \boldsymbol{T} \boldsymbol{B}) \operatorname{tr}(\boldsymbol{T C})]=O\left(a^{2} p^{3}\right), \\
& \mathrm{E}[\operatorname{tr}(\boldsymbol{T} \boldsymbol{A}) \operatorname{tr}(\boldsymbol{T} \boldsymbol{B}) \operatorname{tr}(\boldsymbol{T C})]=O\left(a^{3} p^{3}\right)
\end{aligned}
$$

as $p \rightarrow \infty$ with (4.1) for $p \times p$ matrices $\boldsymbol{A}, \boldsymbol{B}$, and $\boldsymbol{C}$ which satisfy

$$
\begin{gathered}
\operatorname{tr}\left((\boldsymbol{\Sigma} \boldsymbol{A})^{4}\right)=O(p), \operatorname{tr}\left((\boldsymbol{\Sigma} \boldsymbol{B})^{4}\right)=O(p), \operatorname{tr}\left((\boldsymbol{\Sigma} \boldsymbol{C})^{4}\right)=O(p), \\
\operatorname{tr}(\boldsymbol{\Sigma} \boldsymbol{A} \boldsymbol{\Sigma} \boldsymbol{B} \boldsymbol{\Sigma} \boldsymbol{C})=O(p), \operatorname{tr}\left(\boldsymbol{\Sigma} \boldsymbol{A}^{\prime} \boldsymbol{\Sigma} \boldsymbol{B} \boldsymbol{\Sigma} \boldsymbol{C}\right)=O(p), \\
\operatorname{tr}\left(\boldsymbol{\Sigma} \boldsymbol{B}^{\prime} \boldsymbol{\Sigma} \boldsymbol{A}^{\prime} \boldsymbol{\Sigma} \boldsymbol{C}\right)=O(p), \operatorname{tr}\left(\boldsymbol{\Sigma} \boldsymbol{A} \boldsymbol{\Sigma} \boldsymbol{B}^{\prime} \boldsymbol{\Sigma} \boldsymbol{C}\right)=O(p), \\
\operatorname{tr}\left(\boldsymbol{\Sigma} \boldsymbol{A} \boldsymbol{\Sigma} \boldsymbol{B} \boldsymbol{\Sigma} \boldsymbol{C}^{\prime}\right)=O(p), \operatorname{tr}(\boldsymbol{\Sigma} \boldsymbol{A} \boldsymbol{\Sigma} \boldsymbol{C} \boldsymbol{\Sigma} \boldsymbol{B})=O(p), \operatorname{tr}\left(\boldsymbol{\Sigma} \boldsymbol{A} \boldsymbol{\Sigma} \boldsymbol{C}^{\prime} \boldsymbol{\Sigma} \boldsymbol{B}\right)=O(p), \\
\operatorname{tr}(\boldsymbol{\Sigma} \boldsymbol{A} \boldsymbol{\Sigma} \boldsymbol{B})=O(p), \operatorname{tr}(\boldsymbol{\Sigma} \boldsymbol{A} \boldsymbol{\Sigma} \boldsymbol{C})=O(p), \operatorname{tr}(\boldsymbol{\Sigma} \boldsymbol{B} \boldsymbol{\Sigma} \boldsymbol{C})=O(p), \\
\operatorname{tr}\left(\boldsymbol{\Sigma} \boldsymbol{A}^{\prime} \boldsymbol{\Sigma} \boldsymbol{B}\right)=O(p), \operatorname{tr}\left(\boldsymbol{\Sigma} \boldsymbol{A}^{\prime} \boldsymbol{\Sigma} \boldsymbol{C}\right)=O(p), \operatorname{tr}\left(\boldsymbol{\Sigma} \boldsymbol{B}^{\prime} \boldsymbol{\Sigma} \boldsymbol{C}\right)=O(p), \\
\operatorname{tr}(\boldsymbol{\Sigma} \boldsymbol{A})=O(p), \operatorname{tr}(\boldsymbol{\Sigma} \boldsymbol{B})=O(p), \operatorname{tr}(\boldsymbol{\Sigma} \boldsymbol{C})=O(p) .
\end{gathered}
$$

Proof Let $\left\{\boldsymbol{z}_{i}\right\}_{i=1}^{a}$ be an iid sequence satisfying $\boldsymbol{z}_{1}, \ldots, \boldsymbol{z}_{a} \sim N_{p}(\mathbf{0}, \boldsymbol{\Sigma})$. We see that

$$
\begin{aligned}
& \sum_{i=1}^{a} \mathrm{E}\left[\left(\boldsymbol{z}_{i}^{\prime} \boldsymbol{A} \boldsymbol{z}_{i}\right)\left(\boldsymbol{z}_{i}^{\prime} \boldsymbol{B} \boldsymbol{z}_{i}\right)\left(\boldsymbol{z}_{i}^{\prime} \boldsymbol{C} \boldsymbol{z}_{i}\right)\right]=a \mathrm{E}\left[\left(\boldsymbol{z}_{1}^{\prime} \boldsymbol{A} \boldsymbol{z}_{1}\right)\left(\boldsymbol{z}_{1}^{\prime} \boldsymbol{B} \boldsymbol{z}_{1}\right)\left(\boldsymbol{z}_{1}^{\prime} \boldsymbol{C} \boldsymbol{z}_{1}\right)\right] \\
& \quad \leq a\left(\mathrm{E}\left[\left(\boldsymbol{z}_{1}^{\prime} \boldsymbol{A} \boldsymbol{z}_{1}\right)^{2}\left(\boldsymbol{z}_{1}^{\prime} \boldsymbol{B} \boldsymbol{z}_{1}\right)^{2}\right] \mathrm{E}\left[\left(\boldsymbol{z}_{1}^{\prime} \boldsymbol{C} \boldsymbol{z}_{1}\right)^{2}\right]\right)^{\frac{1}{2}} \\
& \quad \leq a\left(\mathrm{E}\left[\left(\boldsymbol{z}_{1}^{\prime} \boldsymbol{A} \boldsymbol{z}_{1}\right)^{4}\right] \mathrm{E}\left[\left(\boldsymbol{z}_{1}^{\prime} \boldsymbol{B} \boldsymbol{z}_{1}\right)^{4}\right] \mathrm{E}\left[\left(\boldsymbol{z}_{1}^{\prime} \boldsymbol{C} \boldsymbol{z}_{1}\right)^{4}\right]\right)^{\frac{1}{4}} \\
& \quad=O\left(a p^{3}\right)
\end{aligned}
$$

where $\mathrm{E}\left[\left(\boldsymbol{z}_{1}^{\prime} \boldsymbol{A} \boldsymbol{z}_{1}\right)^{4}\right]=O\left(p^{4}\right), \mathrm{E}\left[\left(\boldsymbol{z}_{1}^{\prime} \boldsymbol{B} \boldsymbol{z}_{1}\right)^{4}\right]=O\left(p^{4}\right)$, and $\mathrm{E}\left[\left(\boldsymbol{z}_{1}^{\prime} \boldsymbol{C} \boldsymbol{z}_{1}\right)^{4}\right]=O\left(p^{4}\right)$ follow from Lemma 4.1 and $2 \boldsymbol{z}_{1}^{\prime} \boldsymbol{A} \boldsymbol{z}_{1}=$ 
$\boldsymbol{z}_{1}^{\prime}\left(\boldsymbol{A}+\boldsymbol{A}^{\prime}\right) \boldsymbol{z}_{1}$. Then, we have

$$
\begin{aligned}
& \mathrm{E}[\operatorname{tr}(\boldsymbol{T} \boldsymbol{A} \boldsymbol{T} \boldsymbol{B} \boldsymbol{T} \boldsymbol{C})] \\
& =\operatorname{tr}\left(\mathrm{E}\left[\left(\sum_{i=1}^{a} \boldsymbol{z}_{i} \boldsymbol{z}_{i}^{\prime}\right) \boldsymbol{A}\left(\sum_{j=1}^{a} \boldsymbol{z}_{j} \boldsymbol{z}_{j}^{\prime}\right) \boldsymbol{B}\left(\sum_{k=1}^{a} \boldsymbol{z}_{k} \boldsymbol{z}_{k}^{\prime}\right) \boldsymbol{C}\right]\right) \\
& =\operatorname{tr}\left(\mathrm{E}\left[\sum_{i=1}^{a} \sum_{j=1}^{a} \sum_{k=1}^{a} \boldsymbol{z}_{i} \boldsymbol{z}_{i}^{\prime} \boldsymbol{A} \boldsymbol{z}_{j} \boldsymbol{z}_{j}^{\prime} \boldsymbol{B} \boldsymbol{z}_{k} \boldsymbol{z}_{k}^{\prime} \boldsymbol{C}\right]\right) \\
& =\operatorname{tr}\left(\mathrm{E}\left[\sum_{i=1}^{a} \boldsymbol{z}_{i} \boldsymbol{z}_{i}^{\prime} \boldsymbol{A} \boldsymbol{z}_{i} \boldsymbol{z}_{i}^{\prime} \boldsymbol{B} \boldsymbol{z}_{i} \boldsymbol{z}_{i}^{\prime} \boldsymbol{C}\right]+\mathrm{E}\left[\sum_{i=1}^{a} \sum_{k \neq i} \boldsymbol{z}_{i} \boldsymbol{z}_{i}^{\prime} \boldsymbol{A} \boldsymbol{z}_{i} \boldsymbol{z}_{i}^{\prime} \boldsymbol{B} \boldsymbol{z}_{k} \boldsymbol{z}_{k}^{\prime} \boldsymbol{C}\right]+\mathrm{E}\left[\sum_{i=1}^{a} \sum_{j \neq i} \boldsymbol{z}_{i} \boldsymbol{z}_{i}^{\prime} \boldsymbol{A} \boldsymbol{z}_{j} \boldsymbol{z}_{j}^{\prime} \boldsymbol{B} \boldsymbol{z}_{i} \boldsymbol{z}_{i}^{\prime} \boldsymbol{C}\right]\right. \\
& \left.+\mathrm{E}\left[\sum_{i=1}^{a} \sum_{j \neq i} \boldsymbol{z}_{i} \boldsymbol{z}_{i}^{\prime} \boldsymbol{A} \boldsymbol{z}_{j} \boldsymbol{z}_{j}^{\prime} \boldsymbol{B} \boldsymbol{z}_{j} \boldsymbol{z}_{j}^{\prime} \boldsymbol{C}\right]+\mathrm{E}\left[\sum_{i=1}^{a} \sum_{j \neq i} \sum_{k \neq i, j} \boldsymbol{z}_{i} \boldsymbol{z}_{i}^{\prime} \boldsymbol{A} \boldsymbol{z}_{j} \boldsymbol{z}_{j}^{\prime} \boldsymbol{B} \boldsymbol{z}_{k} \boldsymbol{z}_{k}^{\prime} \boldsymbol{C}\right]\right) \\
& =\sum_{i=1}^{a} \mathrm{E}\left[\left(\boldsymbol{z}_{i}^{\prime} \boldsymbol{A} \boldsymbol{z}_{i}\right)\left(\boldsymbol{z}_{i}^{\prime} \boldsymbol{B} \boldsymbol{z}_{i}\right)\left(\boldsymbol{z}_{i}^{\prime} \boldsymbol{C} \boldsymbol{z}_{i}\right)\right]+\operatorname{tr}\left(\sum_{i=1}^{a} \sum_{k \neq i}\left\{\left(\boldsymbol{\Sigma} \boldsymbol{A} \boldsymbol{\Sigma}+\boldsymbol{\Sigma} \boldsymbol{A}^{\prime} \boldsymbol{\Sigma}+\operatorname{tr}(\boldsymbol{\Sigma} \boldsymbol{A}) \boldsymbol{\Sigma}\right) \boldsymbol{B} \boldsymbol{\Sigma} \boldsymbol{C}\right\}\right. \\
& +\sum_{i=1}^{a} \sum_{j \neq i}\left\{\left(\boldsymbol{\Sigma} \boldsymbol{A} \boldsymbol{\Sigma} \boldsymbol{B} \boldsymbol{\Sigma}+\boldsymbol{\Sigma} \boldsymbol{B}^{\prime} \boldsymbol{\Sigma} \boldsymbol{A}^{\prime} \boldsymbol{\Sigma}+\operatorname{tr}(\boldsymbol{\Sigma} \boldsymbol{A} \boldsymbol{\Sigma} \boldsymbol{B}) \boldsymbol{\Sigma}\right) \boldsymbol{C}\right\} \\
& \left.+\sum_{i=1}^{a} \sum_{j \neq i}\left\{\boldsymbol{\Sigma} \boldsymbol{A}\left(\boldsymbol{\Sigma} \boldsymbol{B} \boldsymbol{\Sigma}+\boldsymbol{\Sigma} \boldsymbol{B}^{\prime} \boldsymbol{\Sigma}+\operatorname{tr}(\boldsymbol{\Sigma} \boldsymbol{B}) \boldsymbol{\Sigma}\right) \boldsymbol{C}\right\}+\sum_{i=1}^{a} \sum_{j \neq i} \sum_{k \neq i, j}\{\boldsymbol{\Sigma} \boldsymbol{A} \boldsymbol{\Sigma} \boldsymbol{B} \boldsymbol{\Sigma} \boldsymbol{C}\}\right) \\
& =O\left(a p^{3}\right)+O\left(a^{2} p^{2}\right)+O\left(a^{2} p^{2}\right)+O\left(a^{2} p^{2}\right)+O\left(a^{3} p\right) \\
& =O\left(a p^{3}\right) \text {. }
\end{aligned}
$$

The second assertion follows from

$$
\begin{aligned}
& \mathrm{E}[\operatorname{tr}(\boldsymbol{T} \boldsymbol{A} \boldsymbol{T} \boldsymbol{B}) \operatorname{tr}(\boldsymbol{T} \boldsymbol{C})] \\
& =\mathrm{E}\left[\operatorname{tr}\left(\left(\sum_{i=1}^{a} \boldsymbol{z}_{i} \boldsymbol{z}_{i}^{\prime}\right) \boldsymbol{A}\left(\sum_{j=1}^{a} \boldsymbol{z}_{j} \boldsymbol{z}_{j}^{\prime}\right) \boldsymbol{B}\right) \operatorname{tr}\left(\left(\sum_{k=1}^{a} \boldsymbol{z}_{k} \boldsymbol{z}_{k}^{\prime}\right) \boldsymbol{C}\right)\right] \\
& =\mathrm{E}\left[\sum_{i=1}^{a} \sum_{j=1}^{a} \sum_{k=1}^{a} \operatorname{tr}\left(\boldsymbol{z}_{i} \boldsymbol{z}_{i}^{\prime} \boldsymbol{A} \boldsymbol{z}_{j} \boldsymbol{z}_{j}^{\prime} \boldsymbol{B}\right) \operatorname{tr}\left(\boldsymbol{z}_{k} \boldsymbol{z}_{k}^{\prime} \boldsymbol{C}\right)\right] \\
& =\mathrm{E}\left[\sum_{i=1}^{a} \operatorname{tr}\left(\boldsymbol{z}_{i} \boldsymbol{z}_{i}^{\prime} \boldsymbol{A} \boldsymbol{z}_{i} \boldsymbol{z}_{i}^{\prime} \boldsymbol{B}\right) \operatorname{tr}\left(\boldsymbol{z}_{i} \boldsymbol{z}_{i}^{\prime} \boldsymbol{C}\right)\right]+\mathrm{E}\left[\sum_{i=1}^{a} \sum_{k \neq i} \operatorname{tr}\left(\boldsymbol{z}_{i} \boldsymbol{z}_{i}^{\prime} \boldsymbol{A} \boldsymbol{z}_{i} \boldsymbol{z}_{i}^{\prime} \boldsymbol{B}\right) \operatorname{tr}\left(\boldsymbol{z}_{k} \boldsymbol{z}_{k}^{\prime} \boldsymbol{C}\right)\right] \\
& +\mathrm{E}\left[\sum_{i=1}^{a} \sum_{j \neq i} \operatorname{tr}\left(\boldsymbol{z}_{i} \boldsymbol{z}_{i}^{\prime} \boldsymbol{A} \boldsymbol{z}_{j} \boldsymbol{z}_{j}^{\prime} \boldsymbol{B}\right) \operatorname{tr}\left(\boldsymbol{z}_{i} \boldsymbol{z}_{i}^{\prime} \boldsymbol{C}\right)\right]+\mathrm{E}\left[\sum_{i=1}^{a} \sum_{j \neq i} \operatorname{tr}\left(\boldsymbol{z}_{i} \boldsymbol{z}_{i}^{\prime} \boldsymbol{A} \boldsymbol{z}_{j} \boldsymbol{z}_{j}^{\prime} \boldsymbol{B}\right) \operatorname{tr}\left(\boldsymbol{z}_{j} \boldsymbol{z}_{j}^{\prime} \boldsymbol{C}\right)\right] \\
& +\mathrm{E}\left[\sum_{i=1}^{a} \sum_{j \neq i} \sum_{k \neq i, j} \operatorname{tr}\left(\boldsymbol{z}_{i} \boldsymbol{z}_{i}^{\prime} \boldsymbol{A} \boldsymbol{z}_{j} \boldsymbol{z}_{j}^{\prime} \boldsymbol{B}\right) \operatorname{tr}\left(\boldsymbol{z}_{k} \boldsymbol{z}_{k}^{\prime} \boldsymbol{C}\right)\right] \\
& =\mathrm{E}\left[\sum_{i=1}^{a}\left(\boldsymbol{z}_{i}^{\prime} \boldsymbol{A} \boldsymbol{z}_{i}\right)\left(\boldsymbol{z}_{i}^{\prime} \boldsymbol{B} \boldsymbol{z}_{i}\right)\left(\boldsymbol{z}_{i}^{\prime} \boldsymbol{C} \boldsymbol{z}_{i}\right)\right]+\sum_{i=1}^{a} \sum_{k \neq i} \operatorname{tr}\left(\left(\boldsymbol{\Sigma} \boldsymbol{A} \boldsymbol{\Sigma}+\boldsymbol{\Sigma} \boldsymbol{A}^{\prime} \boldsymbol{\Sigma}+\operatorname{tr}(\boldsymbol{\Sigma} \boldsymbol{A}) \boldsymbol{\Sigma}\right) \boldsymbol{B}\right) \operatorname{tr}(\boldsymbol{\Sigma} \boldsymbol{C}) \\
& +\sum_{i=1}^{a} \sum_{j \neq i} \operatorname{tr}\left(\left(\boldsymbol{\Sigma} \boldsymbol{\Sigma} \boldsymbol{\Sigma}+\boldsymbol{\Sigma} \boldsymbol{C}^{\prime} \boldsymbol{\Sigma}+\operatorname{tr}(\boldsymbol{\Sigma} \boldsymbol{C}) \boldsymbol{\Sigma}\right) \boldsymbol{A} \boldsymbol{\Sigma} \boldsymbol{B}\right)+\sum_{i=1}^{a} \sum_{j \neq i} \operatorname{tr}\left(\boldsymbol{\Sigma} \boldsymbol{A}\left(\boldsymbol{\Sigma} \boldsymbol{C} \boldsymbol{\Sigma}+\boldsymbol{\Sigma} \boldsymbol{C}^{\prime} \boldsymbol{\Sigma}+\operatorname{tr}(\boldsymbol{\Sigma} \boldsymbol{C}) \boldsymbol{\Sigma}\right) \boldsymbol{B}\right) \\
& +\sum_{i=1}^{a} \sum_{j \neq i} \sum_{k \neq i, j} \operatorname{tr}(\boldsymbol{\Sigma} \boldsymbol{A} \boldsymbol{\Sigma} \boldsymbol{B}) \operatorname{tr}(\boldsymbol{\Sigma} \boldsymbol{C}) \\
& =O\left(a p^{3}\right)+O\left(a^{2} p^{3}\right)+O\left(a^{2} p^{2}\right)+O\left(a^{2} p^{2}\right)+O\left(a^{3} p^{2}\right) \\
& =O\left(a^{2} p^{3}\right) \text {. }
\end{aligned}
$$


The last assertion follows from

$$
\begin{aligned}
& \mathrm{E}[\operatorname{tr}(\boldsymbol{T} \boldsymbol{A}) \operatorname{tr}(\boldsymbol{T} \boldsymbol{B}) \operatorname{tr}(\boldsymbol{T C})] \\
& =\mathrm{E}\left[\operatorname{tr}\left(\left(\sum_{i=1}^{a} \boldsymbol{z}_{i} \boldsymbol{z}_{i}^{\prime}\right) \boldsymbol{A}\right) \operatorname{tr}\left(\left(\sum_{j=1}^{a} \boldsymbol{z}_{j} \boldsymbol{z}_{j}^{\prime}\right) \boldsymbol{B}\right) \operatorname{tr}\left(\left(\sum_{k=1}^{a} \boldsymbol{z}_{k} \boldsymbol{z}_{k}^{\prime}\right) \boldsymbol{C}\right)\right] \\
& =\mathrm{E}\left[\sum_{i=1}^{a} \sum_{j=1}^{a} \sum_{k=1}^{a} \operatorname{tr}\left(\boldsymbol{z}_{i} \boldsymbol{z}_{i}^{\prime} \boldsymbol{A}\right) \operatorname{tr}\left(\boldsymbol{z}_{j} \boldsymbol{z}_{j}^{\prime} \boldsymbol{B}\right) \operatorname{tr}\left(\boldsymbol{z}_{k} \boldsymbol{z}_{k}^{\prime} \boldsymbol{C}\right)\right] \\
& =\mathrm{E}\left[\sum_{i=1}^{a} \operatorname{tr}\left(\boldsymbol{z}_{i} \boldsymbol{z}_{i}^{\prime} \boldsymbol{A}\right) \operatorname{tr}\left(\boldsymbol{z}_{i} \boldsymbol{z}_{i}^{\prime} \boldsymbol{B}\right) \operatorname{tr}\left(\boldsymbol{z}_{i} \boldsymbol{z}_{i}^{\prime} \boldsymbol{C}\right)\right]+\mathrm{E}\left[\sum_{i=1}^{a} \sum_{k \neq i} \operatorname{tr}\left(\boldsymbol{z}_{i} \boldsymbol{z}_{i}^{\prime} \boldsymbol{A}\right) \operatorname{tr}\left(\boldsymbol{z}_{i} \boldsymbol{z}_{i}^{\prime} \boldsymbol{B}\right) \operatorname{tr}\left(\boldsymbol{z}_{k} \boldsymbol{z}_{k}^{\prime} \boldsymbol{C}\right)\right] \\
& +\mathrm{E}\left[\sum_{i=1}^{a} \sum_{j \neq i} \operatorname{tr}\left(\boldsymbol{z}_{i} \boldsymbol{z}_{i}^{\prime} \boldsymbol{A}\right) \operatorname{tr}\left(\boldsymbol{z}_{j} \boldsymbol{z}_{j}^{\prime} \boldsymbol{B}\right) \operatorname{tr}\left(\boldsymbol{z}_{i} \boldsymbol{z}_{i}^{\prime} \boldsymbol{C}\right)\right]+\mathrm{E}\left[\sum_{i=1}^{a} \sum_{j \neq i} \operatorname{tr}\left(\boldsymbol{z}_{i} \boldsymbol{z}_{i}^{\prime} \boldsymbol{A}\right) \operatorname{tr}\left(\boldsymbol{z}_{j} \boldsymbol{z}_{j}^{\prime} \boldsymbol{B}\right) \operatorname{tr}\left(\boldsymbol{z}_{j} \boldsymbol{z}_{j}^{\prime} \boldsymbol{C}\right)\right] \\
& +\mathrm{E}\left[\sum_{i=1}^{a} \sum_{j \neq i} \sum_{k \neq i, j} \operatorname{tr}\left(\boldsymbol{z}_{i} \boldsymbol{z}_{i}^{\prime} \boldsymbol{A}\right) \operatorname{tr}\left(\boldsymbol{z}_{j} \boldsymbol{z}_{j}^{\prime} \boldsymbol{B}\right) \operatorname{tr}\left(\boldsymbol{z}_{k} \boldsymbol{z}_{k}^{\prime} \boldsymbol{C}\right)\right] \\
& =\mathrm{E}\left[\sum_{i=1}^{a}\left(\boldsymbol{z}_{i}^{\prime} \boldsymbol{A} \boldsymbol{z}_{i}\right)\left(\boldsymbol{z}_{i}^{\prime} \boldsymbol{B} \boldsymbol{z}_{i}\right)\left(\boldsymbol{z}_{i}^{\prime} \boldsymbol{C} \boldsymbol{z}_{i}\right)\right]+\sum_{i=1}^{a} \sum_{k \neq i} \operatorname{tr}\left(\left(\boldsymbol{\Sigma} \boldsymbol{A} \boldsymbol{\Sigma}+\boldsymbol{\Sigma} \boldsymbol{A}^{\prime} \boldsymbol{\Sigma}+\operatorname{tr}(\boldsymbol{\Sigma} \boldsymbol{A}) \boldsymbol{\Sigma}\right) \boldsymbol{B}\right) \operatorname{tr}(\boldsymbol{\Sigma} \boldsymbol{C}) \\
& +\sum_{i=1}^{a} \sum_{j \neq i} \operatorname{tr}\left(\left(\boldsymbol{\Sigma} \boldsymbol{A} \boldsymbol{\Sigma}+\boldsymbol{\Sigma} \boldsymbol{A}^{\prime} \boldsymbol{\Sigma}+\operatorname{tr}(\boldsymbol{\Sigma} \boldsymbol{A}) \boldsymbol{\Sigma}\right) \boldsymbol{C}\right) \operatorname{tr}(\boldsymbol{\Sigma} \boldsymbol{B})+\sum_{i=1}^{a} \sum_{j \neq i} \operatorname{tr}\left(\left(\boldsymbol{\Sigma} \boldsymbol{B} \boldsymbol{\Sigma}+\boldsymbol{\Sigma} \boldsymbol{B}^{\prime} \boldsymbol{\Sigma}+\operatorname{tr}(\boldsymbol{\Sigma} \boldsymbol{B}) \boldsymbol{\Sigma}\right) \boldsymbol{C}\right) \operatorname{tr}(\boldsymbol{\Sigma} \boldsymbol{A}) \\
& +\sum_{i=1}^{a} \sum_{j \neq i} \sum_{k \neq i, j} \operatorname{tr}(\boldsymbol{\Sigma} \boldsymbol{A}) \operatorname{tr}(\boldsymbol{\Sigma} \boldsymbol{B}) \operatorname{tr}(\boldsymbol{\Sigma} \boldsymbol{C}) \\
& =O\left(a p^{3}\right)+O\left(a^{2} p^{3}\right)+O\left(a^{2} p^{3}\right)+O\left(a^{2} p^{3}\right)+O\left(a^{3} p^{3}\right) \\
& =O\left(a^{3} p^{3}\right) \text {. }
\end{aligned}
$$

This completes the proof.

Proposition 4.6. If $\boldsymbol{T} \sim W_{p}(a, \boldsymbol{\Sigma})$ then

$$
\begin{aligned}
& \mathrm{E}[\operatorname{tr}(\boldsymbol{T} \boldsymbol{A T} \boldsymbol{B} \boldsymbol{T C T} \boldsymbol{D})]=O\left(a p^{4}\right), \\
& \mathrm{E}[\operatorname{tr}(\boldsymbol{T} \boldsymbol{A} \boldsymbol{T} \boldsymbol{B} \boldsymbol{T C}) \operatorname{tr}(\boldsymbol{T} \boldsymbol{D})]=O\left(a^{2} p^{4}\right), \\
& \mathrm{E}[\operatorname{tr}(\boldsymbol{T} \boldsymbol{A T} \boldsymbol{B}) \operatorname{tr}(\boldsymbol{T C} \boldsymbol{T} \boldsymbol{D})]=O\left(a^{2} p^{4}\right), \\
& \mathrm{E}[\operatorname{tr}(\boldsymbol{T} \boldsymbol{A} \boldsymbol{T} \boldsymbol{B}) \operatorname{tr}(\boldsymbol{T C}) \operatorname{tr}(\boldsymbol{T} \boldsymbol{D})]=O\left(a^{3} p^{4}\right), \\
& \mathrm{E}[\operatorname{tr}(\boldsymbol{T} \boldsymbol{A}) \operatorname{tr}(\boldsymbol{T} \boldsymbol{B}) \operatorname{tr}(\boldsymbol{T} \boldsymbol{C}) \operatorname{tr}(\boldsymbol{T} \boldsymbol{D})]=O\left(a^{4} p^{4}\right)
\end{aligned}
$$

as $p \rightarrow \infty$ with (4.1) for $p \times p$ matrices $\boldsymbol{A}, \boldsymbol{B}, \boldsymbol{C}$, and $\boldsymbol{D}$ which satisfy

$$
\begin{gathered}
\operatorname{tr}\left(\boldsymbol{\Sigma} \boldsymbol{\Psi}_{1} \boldsymbol{\Sigma} \boldsymbol{\Psi}_{2} \boldsymbol{\Sigma} \boldsymbol{\Psi}_{3} \boldsymbol{\Sigma} \boldsymbol{\Psi}_{4}\right)=O(p), \boldsymbol{\Psi}_{1}, \boldsymbol{\Psi}_{2}, \boldsymbol{\Psi}_{3}, \boldsymbol{\Psi}_{4}=\boldsymbol{A}, \boldsymbol{A}^{\prime}, \boldsymbol{B}, \boldsymbol{B}^{\prime}, \boldsymbol{C}, \boldsymbol{C}^{\prime}, \boldsymbol{D}, \boldsymbol{D}^{\prime} \\
\operatorname{tr}\left(\boldsymbol{\Sigma} \boldsymbol{\Psi}_{1} \boldsymbol{\Sigma} \boldsymbol{\Psi}_{2} \boldsymbol{\Sigma} \boldsymbol{\Psi}_{3}\right)=O(p), \boldsymbol{\Psi}_{1}, \boldsymbol{\Psi}_{2}, \boldsymbol{\Psi}_{3}=\boldsymbol{A}, \boldsymbol{A}^{\prime}, \boldsymbol{B}, \boldsymbol{B}^{\prime}, \boldsymbol{C}, \boldsymbol{C}^{\prime}, \boldsymbol{D}, \boldsymbol{D}^{\prime} \\
\operatorname{tr}\left(\boldsymbol{\Sigma} \boldsymbol{\Psi}_{1} \boldsymbol{\Sigma} \boldsymbol{\Psi}_{2}\right)=O(p), \boldsymbol{\Psi}_{1}, \boldsymbol{\Psi}_{2}=\boldsymbol{A}, \boldsymbol{A}^{\prime}, \boldsymbol{B}, \boldsymbol{B}^{\prime}, \boldsymbol{C}, \boldsymbol{C}^{\prime}, \boldsymbol{D}, \boldsymbol{D}^{\prime} \\
\operatorname{tr}\left(\boldsymbol{\Sigma} \boldsymbol{\Psi}_{1}\right)=O(p), \boldsymbol{\Psi}_{1}=\boldsymbol{A}, \boldsymbol{B}, \boldsymbol{C}, \boldsymbol{D}
\end{gathered}
$$

Proof. First, we see that

$$
\begin{aligned}
& \sum_{i=1}^{a} \mathrm{E}\left[\left(\boldsymbol{z}_{i}^{\prime} \boldsymbol{A} \boldsymbol{z}_{i}\right)\left(\boldsymbol{z}_{i}^{\prime} \boldsymbol{B} \boldsymbol{z}_{i}\right)\left(\boldsymbol{z}_{i}^{\prime} \boldsymbol{C} \boldsymbol{z}_{i}\right)\left(\boldsymbol{z}_{i}^{\prime} \boldsymbol{D} \boldsymbol{z}_{i}\right)\right]=a \mathrm{E}\left[\left(\boldsymbol{z}_{1}^{\prime} \boldsymbol{A} \boldsymbol{z}_{1}\right)\left(\boldsymbol{z}_{1}^{\prime} \boldsymbol{B} \boldsymbol{z}_{1}\right)\left(\boldsymbol{z}_{1}^{\prime} \boldsymbol{C} \boldsymbol{z}_{1}\right)\left(\boldsymbol{z}_{1}^{\prime} \boldsymbol{D} \boldsymbol{z}_{1}\right)\right] \\
& \quad \leq a\left(\mathrm{E}\left[\left(\boldsymbol{z}_{1}^{\prime} \boldsymbol{A} \boldsymbol{z}_{1}\right)^{2}\left(\boldsymbol{z}_{1}^{\prime} \boldsymbol{B} \boldsymbol{z}_{1}\right)^{2}\right] \mathrm{E}\left[\left(\boldsymbol{z}_{1}^{\prime} \boldsymbol{C} \boldsymbol{z}_{1}\right)^{2}\left(\boldsymbol{z}_{1}^{\prime} \boldsymbol{D} \boldsymbol{z}_{1}\right)^{2}\right]\right)^{\frac{1}{2}} \\
& \quad \leq a\left(\mathrm{E}\left[\left(\boldsymbol{z}_{1}^{\prime} \boldsymbol{A} \boldsymbol{z}_{1}\right)^{4}\right] \mathrm{E}\left[\left(\boldsymbol{z}_{1}^{\prime} \boldsymbol{B} \boldsymbol{z}_{1}\right)^{4}\right] \mathrm{E}\left[\left(\boldsymbol{z}_{1}^{\prime} \boldsymbol{C} \boldsymbol{z}_{1}\right)^{4}\right] \mathrm{E}\left[\left(\boldsymbol{z}_{1}^{\prime} \boldsymbol{D} \boldsymbol{z}_{1}\right)^{4}\right]\right)^{\frac{1}{4}} \\
& \quad=O\left(a p^{4}\right) .
\end{aligned}
$$


Then, we have

$\mathrm{E}[\operatorname{tr}(\boldsymbol{T} \boldsymbol{A} \boldsymbol{T} \boldsymbol{B} \boldsymbol{T} \boldsymbol{C} \boldsymbol{T} \boldsymbol{D})]$

$$
\begin{aligned}
& =\operatorname{tr}\left(\mathrm{E}\left[\left(\sum_{i=1}^{a} \boldsymbol{z}_{i} \boldsymbol{z}_{i}^{\prime}\right) \boldsymbol{A}\left(\sum_{j=1}^{a} \boldsymbol{z}_{j} \boldsymbol{z}_{j}^{\prime}\right) \boldsymbol{B}\left(\sum_{k=1}^{a} \boldsymbol{z}_{k} \boldsymbol{z}_{k}^{\prime}\right) \boldsymbol{C}\left(\sum_{l=1}^{a} \boldsymbol{z}_{l} \boldsymbol{z}_{l}^{\prime}\right) \boldsymbol{D}\right]\right) \\
& =\operatorname{tr}\left(\mathrm{E}\left[\sum_{i=1}^{a} \sum_{j=1}^{a} \sum_{k=1}^{a} \sum_{l=1}^{a} \boldsymbol{z}_{i} \boldsymbol{z}_{i}^{\prime} \boldsymbol{A} \boldsymbol{z}_{j} \boldsymbol{z}_{j}^{\prime} \boldsymbol{B} \boldsymbol{z}_{k} \boldsymbol{z}_{k}^{\prime} \boldsymbol{C} \boldsymbol{z}_{l} \boldsymbol{z}_{l}^{\prime} \boldsymbol{D}\right]\right) \\
& =\operatorname{tr}\left(\mathrm{E}\left[\sum_{i=1}^{a} \boldsymbol{z}_{i} \boldsymbol{z}_{i}^{\prime} \boldsymbol{A} \boldsymbol{z}_{i} \boldsymbol{z}_{i}^{\prime} \boldsymbol{B} \boldsymbol{z}_{i} \boldsymbol{z}_{i}^{\prime} \boldsymbol{C} \boldsymbol{z}_{i} \boldsymbol{z}_{i}^{\prime} \boldsymbol{D}\right]+\mathrm{E}\left[\sum_{i=1}^{a} \sum_{l \neq i} \boldsymbol{z}_{i} \boldsymbol{z}_{i}^{\prime} \boldsymbol{A} \boldsymbol{z}_{i} \boldsymbol{z}_{i}^{\prime} \boldsymbol{B} \boldsymbol{z}_{i} \boldsymbol{z}_{i}^{\prime} \boldsymbol{C} \boldsymbol{z}_{l} \boldsymbol{z}_{l}^{\prime} \boldsymbol{D}\right]+\mathrm{E}\left[\sum_{i=1}^{a} \sum_{k \neq i} \boldsymbol{z}_{i} \boldsymbol{z}_{i}^{\prime} \boldsymbol{A} \boldsymbol{z}_{i} \boldsymbol{z}_{i}^{\prime} \boldsymbol{B} \boldsymbol{z}_{k} \boldsymbol{z}_{k}^{\prime} \boldsymbol{C} \boldsymbol{z}_{i} \boldsymbol{z}_{i}^{\prime} \boldsymbol{D}\right]\right. \\
& +\mathrm{E}\left[\sum_{i=1}^{a} \sum_{j \neq i} \boldsymbol{z}_{i} \boldsymbol{z}_{i}^{\prime} \boldsymbol{A} \boldsymbol{z}_{j} \boldsymbol{z}_{j}^{\prime} \boldsymbol{B} \boldsymbol{z}_{i} \boldsymbol{z}_{i}^{\prime} \boldsymbol{C} \boldsymbol{z}_{i} \boldsymbol{z}_{i}^{\prime} \boldsymbol{D}\right]+\mathrm{E}\left[\sum_{i=1}^{a} \sum_{j \neq i} \boldsymbol{z}_{i} \boldsymbol{z}_{i}^{\prime} \boldsymbol{A} \boldsymbol{z}_{j} \boldsymbol{z}_{j}^{\prime} \boldsymbol{B} \boldsymbol{z}_{j} \boldsymbol{z}_{j}^{\prime} \boldsymbol{C} \boldsymbol{z}_{j} \boldsymbol{z}_{j}^{\prime} \boldsymbol{D}\right]+\mathrm{E}\left[\sum_{i=1}^{a} \sum_{k \neq i} \boldsymbol{z}_{i} \boldsymbol{z}_{i}^{\prime} \boldsymbol{A} \boldsymbol{z}_{i} \boldsymbol{z}_{i}^{\prime} \boldsymbol{B} \boldsymbol{z}_{k} \boldsymbol{z}_{k}^{\prime} \boldsymbol{C} \boldsymbol{z}_{k} \boldsymbol{z}_{k}^{\prime} \boldsymbol{D}\right] \\
& +\mathrm{E}\left[\sum_{i=1}^{a} \sum_{j \neq i} \boldsymbol{z}_{i} \boldsymbol{z}_{i}^{\prime} \boldsymbol{A} \boldsymbol{z}_{j} \boldsymbol{z}_{j}^{\prime} \boldsymbol{B} \boldsymbol{z}_{i} \boldsymbol{z}_{i}^{\prime} \boldsymbol{C} \boldsymbol{z}_{j} \boldsymbol{z}_{j}^{\prime} \boldsymbol{D}\right]+\mathrm{E}\left[\sum_{i=1}^{a} \sum_{j \neq i} \boldsymbol{z}_{i} \boldsymbol{z}_{i}^{\prime} \boldsymbol{A} \boldsymbol{z}_{j} \boldsymbol{z}_{j}^{\prime} \boldsymbol{B} \boldsymbol{z}_{j} \boldsymbol{z}_{j}^{\prime} \boldsymbol{C} \boldsymbol{z}_{i} \boldsymbol{z}_{i}^{\prime} \boldsymbol{D}\right] \\
& +\mathrm{E}\left[\sum_{i=1}^{a} \sum_{k \neq i} \sum_{l \neq i, k} \boldsymbol{z}_{i} \boldsymbol{z}_{i}^{\prime} \boldsymbol{A} \boldsymbol{z}_{i} \boldsymbol{z}_{i}^{\prime} \boldsymbol{B} \boldsymbol{z}_{k} \boldsymbol{z}_{k}^{\prime} \boldsymbol{C} \boldsymbol{z}_{l} \boldsymbol{z}_{l}^{\prime} \boldsymbol{D}\right]+\mathrm{E}\left[\sum_{i=1}^{a} \sum_{j \neq i} \sum_{l \neq i, j} \boldsymbol{z}_{i} \boldsymbol{z}_{i}^{\prime} \boldsymbol{A} \boldsymbol{z}_{j} \boldsymbol{z}_{j}^{\prime} \boldsymbol{B} \boldsymbol{z}_{i} \boldsymbol{z}_{i}^{\prime} \boldsymbol{C} \boldsymbol{z}_{l} \boldsymbol{z}_{l}^{\prime} \boldsymbol{D}\right] \\
& +\mathrm{E}\left[\sum_{i=1}^{a} \sum_{j \neq i} \sum_{k \neq i, j} \boldsymbol{z}_{i} \boldsymbol{z}_{i}^{\prime} \boldsymbol{A} \boldsymbol{z}_{j} \boldsymbol{z}_{j}^{\prime} \boldsymbol{B} \boldsymbol{z}_{k} \boldsymbol{z}_{k}^{\prime} \boldsymbol{C} \boldsymbol{z}_{i} \boldsymbol{z}_{i}^{\prime} \boldsymbol{D}\right]+\mathrm{E}\left[\sum_{i=1}^{a} \sum_{j \neq i} \sum_{l \neq i, j} \boldsymbol{z}_{i} \boldsymbol{z}_{i}^{\prime} \boldsymbol{A} \boldsymbol{z}_{j} \boldsymbol{z}_{j}^{\prime} \boldsymbol{B} \boldsymbol{z}_{j} \boldsymbol{z}_{j}^{\prime} \boldsymbol{C} \boldsymbol{z}_{l} \boldsymbol{z}_{l}^{\prime} \boldsymbol{D}\right] \\
& +\mathrm{E}\left[\sum_{i=1}^{a} \sum_{j \neq i} \sum_{k \neq i, j} \boldsymbol{z}_{i} \boldsymbol{z}_{i}^{\prime} \boldsymbol{A} \boldsymbol{z}_{j} \boldsymbol{z}_{j}^{\prime} \boldsymbol{B} \boldsymbol{z}_{k} \boldsymbol{z}_{k}^{\prime} \boldsymbol{C} \boldsymbol{z}_{j} \boldsymbol{z}_{j}^{\prime} \boldsymbol{D}\right]+\mathrm{E}\left[\sum_{i=1}^{a} \sum_{j \neq i} \sum_{k \neq i, j} \boldsymbol{z}_{i} \boldsymbol{z}_{i}^{\prime} \boldsymbol{A} \boldsymbol{z}_{j} \boldsymbol{z}_{j}^{\prime} \boldsymbol{B} \boldsymbol{z}_{k} \boldsymbol{z}_{k}^{\prime} \boldsymbol{C} \boldsymbol{z}_{k} \boldsymbol{z}_{k}^{\prime} \boldsymbol{D}\right] \\
& \left.+\mathrm{E}\left[\sum_{i=1}^{a} \sum_{j \neq i} \sum_{k \neq i, j} \sum_{l \neq i, j, k} \boldsymbol{z}_{i} \boldsymbol{z}_{i}^{\prime} \boldsymbol{A} \boldsymbol{z}_{j} \boldsymbol{z}_{j}^{\prime} \boldsymbol{B} \boldsymbol{z}_{k} \boldsymbol{z}_{k}^{\prime} \boldsymbol{C} \boldsymbol{z}_{l} \boldsymbol{z}_{l}^{\prime} \boldsymbol{D}\right]\right) \\
& =\mathrm{E}\left[\sum_{i=1}^{a}\left(\boldsymbol{z}_{i}^{\prime} \boldsymbol{A} \boldsymbol{z}_{i}\right)\left(\boldsymbol{z}_{i}^{\prime} \boldsymbol{B} \boldsymbol{z}_{i}\right)\left(\boldsymbol{z}_{i}^{\prime} \boldsymbol{C} \boldsymbol{z}_{i}\right)\left(\boldsymbol{z}_{i}^{\prime} \boldsymbol{D} \boldsymbol{z}_{i}\right)\right]+\mathrm{E}\left[\sum_{i=1}^{a} \sum_{l \neq i}\left(\boldsymbol{z}_{i}^{\prime} \boldsymbol{A} \boldsymbol{z}_{i}\right)\left(\boldsymbol{z}_{i}^{\prime} \boldsymbol{B} \boldsymbol{z}_{i}\right)\left(\boldsymbol{z}_{i}^{\prime} \boldsymbol{C} \boldsymbol{\Sigma} \boldsymbol{D} \boldsymbol{z}_{i}\right)\right] \\
& +\mathrm{E}\left[\sum_{i=1}^{a} \sum_{k \neq i}\left(\boldsymbol{z}_{i}^{\prime} \boldsymbol{A} \boldsymbol{z}_{i}\right)\left(\boldsymbol{z}_{i}^{\prime} \boldsymbol{B} \boldsymbol{\Sigma} \boldsymbol{C} \boldsymbol{z}_{i}\right)\left(\boldsymbol{z}_{i}^{\prime} \boldsymbol{D} \boldsymbol{z}_{i}\right)\right]+\mathrm{E}\left[\sum_{i=1}^{a} \sum_{j \neq i}\left(\boldsymbol{z}_{i}^{\prime} \boldsymbol{A} \boldsymbol{\Sigma} \boldsymbol{B} \boldsymbol{z}_{i}\right)\left(\boldsymbol{z}_{i}^{\prime} \boldsymbol{C} \boldsymbol{z}_{i}\right)\left(\boldsymbol{z}_{i}^{\prime} \boldsymbol{D} \boldsymbol{z}_{i}\right)\right] \\
& +\mathrm{E}\left[\sum_{i=1}^{a} \sum_{j \neq i}\left(\boldsymbol{z}_{j}^{\prime} \boldsymbol{D} \boldsymbol{\Sigma} \boldsymbol{A} \boldsymbol{z}_{j}\right)\left(\boldsymbol{z}_{j}^{\prime} \boldsymbol{B} \boldsymbol{z}_{j}\right)\left(\boldsymbol{z}_{j}^{\prime} \boldsymbol{C} \boldsymbol{z}_{j}\right)\right] \\
& +\mathrm{E}\left[\sum_{i=1}^{a} \sum_{k \neq i} \operatorname{tr}\left\{\left(\boldsymbol{\Sigma} \boldsymbol{A} \boldsymbol{\Sigma} \boldsymbol{B}+\boldsymbol{\Sigma} \boldsymbol{A}^{\prime} \boldsymbol{\Sigma} \boldsymbol{B}+\operatorname{tr}(\boldsymbol{\Sigma} \boldsymbol{A}) \boldsymbol{\Sigma} \boldsymbol{B}\right)\left(\boldsymbol{\Sigma} \boldsymbol{C} \boldsymbol{\Sigma} \boldsymbol{D}+\boldsymbol{\Sigma} \boldsymbol{C}^{\prime} \boldsymbol{\Sigma} \boldsymbol{D}+\operatorname{tr}(\boldsymbol{\Sigma} \boldsymbol{C}) \boldsymbol{\Sigma} \boldsymbol{D}\right)\right\}\right] \\
& +\mathrm{E}\left[\sum_{i=1}^{a} \sum_{j \neq i} \operatorname{tr}\left\{\left(\boldsymbol{\Sigma} \boldsymbol{A} \boldsymbol{z}_{j} \boldsymbol{z}_{j}^{\prime} \boldsymbol{B} \boldsymbol{\Sigma} \boldsymbol{C}+\boldsymbol{\Sigma} \boldsymbol{B}^{\prime} \boldsymbol{z}_{j} \boldsymbol{z}_{j}^{\prime} \boldsymbol{A}^{\prime} \boldsymbol{\Sigma} \boldsymbol{C}+\operatorname{tr}\left(\boldsymbol{\Sigma} \boldsymbol{A} \boldsymbol{z}_{j} \boldsymbol{z}_{j}^{\prime} \boldsymbol{B}\right) \boldsymbol{\Sigma} \boldsymbol{C}\right) \boldsymbol{z}_{j} \boldsymbol{z}_{j}^{\prime} \boldsymbol{D}\right\}\right] \\
& +\mathrm{E}\left[\sum_{i=1}^{a} \sum_{j \neq i} \operatorname{tr}\left\{\left(\boldsymbol{\Sigma} \boldsymbol{D} \boldsymbol{\Sigma} \boldsymbol{A}+\boldsymbol{\Sigma} \boldsymbol{D}^{\prime} \boldsymbol{\Sigma} \boldsymbol{A}+\operatorname{tr}(\boldsymbol{\Sigma} \boldsymbol{D}) \boldsymbol{\Sigma} \boldsymbol{A}\right)\left(\boldsymbol{\Sigma} \boldsymbol{B} \boldsymbol{\Sigma} \boldsymbol{C}+\boldsymbol{\Sigma} \boldsymbol{B}^{\prime} \boldsymbol{\Sigma} \boldsymbol{C}+\operatorname{tr}(\boldsymbol{\Sigma} \boldsymbol{B}) \boldsymbol{\Sigma} \boldsymbol{C}\right)\right\}\right] \\
& +\mathrm{E}\left[\sum_{i=1}^{a} \sum_{k \neq i} \sum_{l \neq i, k} \boldsymbol{z}_{i}^{\prime} \boldsymbol{A} \boldsymbol{z}_{i} \boldsymbol{z}_{i}^{\prime} \boldsymbol{B} \boldsymbol{\Sigma} \boldsymbol{C} \boldsymbol{\Sigma} \boldsymbol{D} \boldsymbol{z}_{i}\right]+\mathrm{E}\left[\sum_{i=1}^{a} \sum_{j \neq i} \sum_{l \neq i, j} \boldsymbol{z}_{i}^{\prime} \boldsymbol{A} \boldsymbol{\Sigma} \boldsymbol{B} \boldsymbol{z}_{i} \boldsymbol{z}_{i}^{\prime} \boldsymbol{C} \boldsymbol{\Sigma} \boldsymbol{D} \boldsymbol{z}_{i}\right] \\
& +\mathrm{E}\left[\sum_{i=1}^{a} \sum_{j \neq i} \sum_{k \neq i, j} \boldsymbol{z}_{i}^{\prime} \boldsymbol{A} \boldsymbol{\Sigma} \boldsymbol{B} \boldsymbol{\Sigma} \boldsymbol{C} \boldsymbol{z}_{i} \boldsymbol{z}_{i}^{\prime} \boldsymbol{D} \boldsymbol{z}_{i}\right]+\mathrm{E}\left[\sum_{i=1}^{a} \sum_{j \neq i} \sum_{l \neq i, j} \boldsymbol{z}_{j}^{\prime} \boldsymbol{B} \boldsymbol{z}_{j} \boldsymbol{z}_{j}^{\prime} \boldsymbol{C} \boldsymbol{\Sigma} \boldsymbol{D} \boldsymbol{\Sigma} \boldsymbol{A} \boldsymbol{z}_{j}\right]
\end{aligned}
$$




$$
\begin{aligned}
+ & \mathrm{E}\left[\sum_{i=1}^{a} \sum_{j \neq i} \sum_{k \neq i, j} \boldsymbol{z}_{j}^{\prime} \boldsymbol{B} \boldsymbol{\Sigma} \boldsymbol{C} \boldsymbol{z}_{j} \boldsymbol{z}_{j}^{\prime} \boldsymbol{D} \boldsymbol{\Sigma} \boldsymbol{A} \boldsymbol{z}_{j}\right]+\mathrm{E}\left[\sum_{i=1}^{a} \sum_{j \neq i} \sum_{k \neq i, j} \boldsymbol{z}_{k}^{\prime} \boldsymbol{C} \boldsymbol{z}_{k} \boldsymbol{z}_{k}^{\prime} \boldsymbol{D} \boldsymbol{\Sigma} \boldsymbol{A} \boldsymbol{\Sigma} \boldsymbol{B} \boldsymbol{z}_{k}\right] \\
+ & \mathrm{E}\left[\sum_{i=1}^{a} \sum_{j \neq i} \sum_{k \neq i, j} \sum_{l \neq i, j, k} \operatorname{tr}\{\boldsymbol{\Sigma} \boldsymbol{A} \boldsymbol{\Sigma} \boldsymbol{B} \boldsymbol{\Sigma} \boldsymbol{C} \boldsymbol{\Sigma} \boldsymbol{D}\}\right] \\
= & O\left(a p^{4}\right)+\left\{O\left(a^{2} p^{3}\right)+O\left(a^{2} p^{3}\right)+O\left(a^{2} p^{3}\right)+O\left(a^{2} p^{3}\right)\right\} \\
& +\left\{O\left(a^{2} p^{3}\right)+\mathrm{E}\left[\sum_{i=1}^{a} \sum_{j \neq i} \operatorname{tr}\left\{\boldsymbol{\Sigma} \boldsymbol{A} \boldsymbol{z}_{j}^{\prime} \boldsymbol{B} \boldsymbol{\Sigma} \boldsymbol{C} \boldsymbol{z}_{j} \boldsymbol{z}_{j} \boldsymbol{z}_{j}^{\prime} \boldsymbol{D}+\boldsymbol{\Sigma} \boldsymbol{B}^{\prime} \boldsymbol{z}_{j}^{\prime} \boldsymbol{A}^{\prime} \boldsymbol{\Sigma} \boldsymbol{C} \boldsymbol{z}_{j} \boldsymbol{z}_{j} \boldsymbol{z}_{j}^{\prime} \boldsymbol{D}+\boldsymbol{\Sigma} \boldsymbol{C} \boldsymbol{z}_{j}^{\prime} \boldsymbol{B} \boldsymbol{\Sigma} \boldsymbol{A} \boldsymbol{z}_{j} \boldsymbol{z}_{j} \boldsymbol{z}_{j}^{\prime} \boldsymbol{D}\right\}\right]+O\left(a^{2} p^{3}\right)\right\} \\
& +\left\{O\left(a^{3} p^{2}\right)+O\left(a^{3} p^{2}\right)+O\left(a^{3} p^{2}\right)+O\left(a^{3} p^{2}\right)+O\left(a^{3} p^{2}\right)+O\left(a^{3} p^{2}\right)\right\}+O\left(a^{4} p\right) \\
= & O\left(a p^{4}\right)+\left\{O\left(a^{2} p^{3}\right)+O\left(a^{2} p^{3}\right)+O\left(a^{2} p^{3}\right)+O\left(a^{2} p^{3}\right)\right\}+\left\{O\left(a^{2} p^{3}\right)+O\left(a^{2} p^{2}\right)+O\left(a^{2} p^{3}\right)\right\} \\
& +\left\{O\left(a^{3} p^{2}\right)+O\left(a^{3} p^{2}\right)+O\left(a^{3} p^{2}\right)+O\left(a^{3} p^{2}\right)+O\left(a^{3} p^{2}\right)+O\left(a^{3} p^{2}\right)\right\}+O\left(a^{4} p\right) \\
= & O\left(a p^{4}\right) .
\end{aligned}
$$

We can prove the followings in similar ways:

$$
\begin{aligned}
& \mathrm{E}[\operatorname{tr}(\boldsymbol{T} \boldsymbol{A T} \boldsymbol{B} \boldsymbol{T C}) \operatorname{tr}(\boldsymbol{T} \boldsymbol{D})]=O\left(a^{4} p^{2}\right)+O\left(a^{3} p^{3}\right)+O\left(a^{2} p^{4}\right)=O\left(a^{2} p^{4}\right), \\
& \mathrm{E}[\operatorname{tr}(\boldsymbol{T} \boldsymbol{A} \boldsymbol{T} \boldsymbol{B}) \operatorname{tr}(\boldsymbol{T} \boldsymbol{C} \boldsymbol{T} \boldsymbol{D})]=O\left(a^{4} p^{2}\right)+O\left(a^{3} p^{3}\right)+O\left(a^{2} p^{4}\right)=O\left(a^{2} p^{4}\right), \\
& \mathrm{E}[\operatorname{tr}(\boldsymbol{T} \boldsymbol{A} \boldsymbol{T} \boldsymbol{B}) \operatorname{tr}(\boldsymbol{T C}) \operatorname{tr}(\boldsymbol{T} \boldsymbol{D})]=O\left(a^{4} p^{3}\right)+O\left(a^{3} p^{4}\right)=O\left(a^{3} p^{4}\right), \\
& \mathrm{E}[\operatorname{tr}(\boldsymbol{T} \boldsymbol{A}) \operatorname{tr}(\boldsymbol{T} \boldsymbol{B}) \operatorname{tr}(\boldsymbol{T C}) \operatorname{tr}(\boldsymbol{T} \boldsymbol{D})]=O\left(a^{4} p^{4}\right),
\end{aligned}
$$

so we omit their details. This completes the proof.

When applying Propositions 4.4, 4.5, and 4.6, their conditions are not useful. Hence, let us show a simple sufficient condition.

Assumption 3. As $p \rightarrow \infty$ with (4.1), it holds that

$$
\begin{aligned}
& \frac{\operatorname{tr}\left(\boldsymbol{\Sigma}^{2}\right)}{p} \rightarrow \sigma_{2} \in(0, \infty), \\
& \frac{\operatorname{tr}\left(\boldsymbol{\Sigma}^{3}\right)}{p} \rightarrow \sigma_{3} \in(0, \infty), \\
& \vdots \\
& \frac{\operatorname{tr}\left(\boldsymbol{\Sigma}^{16}\right)}{p} \rightarrow \sigma_{16} \in(0, \infty) .
\end{aligned}
$$

The following statement is a direct corollary to Propositions 4.4, 4.5, and 4.6.

Corollary 4.7. Suppose Assumption 3. If $\boldsymbol{T} \sim W_{p}(a, \boldsymbol{\Sigma})$ then the conclusions of Propositions 4.4, 4.5, and 4.6 hold for $\boldsymbol{A}, \boldsymbol{B}, \boldsymbol{C}, \boldsymbol{D}=\boldsymbol{I}_{p}, \boldsymbol{\Sigma}$.

Next, we provide asymptotic evaluations for moments of two Wishart matrices. Before presenting results, we introduce the following condition.

Assumption 4. As $p \rightarrow \infty$ with

$$
a, b \asymp p^{\delta}, \quad 0<\delta<1,
$$

it holds that

$$
\begin{aligned}
& \frac{\operatorname{tr}\left(\boldsymbol{\Sigma}_{i} \boldsymbol{\Sigma}_{j}\right)}{p} \rightarrow \sigma_{i j} \in(0, \infty), \\
& \vdots \\
& \frac{\operatorname{tr}\left(\boldsymbol{\Sigma}_{i} \boldsymbol{\Sigma}_{j} \boldsymbol{\Sigma}_{k} \boldsymbol{\Sigma}_{l} \boldsymbol{\Sigma}_{i^{\prime}}\right)}{p} \rightarrow \sigma_{i j k l i^{\prime}} \in(0, \infty), \\
& \frac{\operatorname{tr}\left(\boldsymbol{\Sigma}_{i} \boldsymbol{\Sigma}_{j} \boldsymbol{\Sigma}_{k} \boldsymbol{\Sigma}_{l} \boldsymbol{\Sigma}_{i^{\prime}} \boldsymbol{\Sigma}_{j^{\prime}}\right)}{p} \rightarrow \sigma_{i j k l i^{\prime} j^{\prime}} \in(-\infty, \infty), \\
& \quad \vdots \quad \frac{\operatorname{tr}\left(\boldsymbol{\Sigma}_{i} \boldsymbol{\Sigma}_{j} \boldsymbol{\Sigma}_{k} \boldsymbol{\Sigma}_{l} \boldsymbol{\Sigma}_{i^{\prime}} \boldsymbol{\Sigma}_{j^{\prime}} \boldsymbol{\Sigma}_{k^{\prime}} \boldsymbol{\Sigma}_{l^{\prime}} \boldsymbol{\Sigma}_{i^{\prime \prime}} \boldsymbol{\Sigma}_{j^{\prime \prime}} \boldsymbol{\Sigma}_{k^{\prime \prime}} \boldsymbol{\Sigma}_{l^{\prime \prime}} \boldsymbol{\Sigma}_{i^{\prime \prime \prime}} \boldsymbol{\Sigma}_{j^{\prime \prime \prime}} \boldsymbol{\Sigma}_{k^{\prime \prime \prime}} \boldsymbol{\Sigma}_{l^{\prime \prime \prime}}\right)}{p} \\
& \rightarrow \sigma_{i j k l i^{\prime} j^{\prime} k^{\prime} l^{\prime} i^{\prime \prime} j^{\prime \prime} k^{\prime \prime} l^{\prime \prime} i^{\prime \prime \prime} j^{\prime \prime \prime} k^{\prime \prime \prime} l^{\prime \prime \prime}} \in(-\infty, \infty)
\end{aligned}
$$


for $i, j, k, l, i^{\prime}, j^{\prime}, k^{\prime}, l^{\prime}, i^{\prime \prime}, j^{\prime \prime}, k^{\prime \prime}, l^{\prime \prime}, i^{\prime \prime \prime}, j^{\prime \prime \prime}, k^{\prime \prime \prime}, l^{\prime \prime \prime}=a, b$.

Proposition 4.8. (i) Under Assumption 4, if $\boldsymbol{T}_{a} \sim W_{p}\left(a, \boldsymbol{\Sigma}_{a}\right)$ and $\boldsymbol{T}_{b} \sim W_{p}\left(b, \boldsymbol{\Sigma}_{b}\right)$ are independent, then

$$
\begin{aligned}
& \mathrm{E}\left[\operatorname{tr}\left(\boldsymbol{T}_{a} \boldsymbol{T}_{a} \boldsymbol{T}_{a} \boldsymbol{T}_{a} \boldsymbol{T}_{b} \boldsymbol{T}_{b} \boldsymbol{T}_{b} \boldsymbol{T}_{b}\right)\right]=O\left(a b p^{7}\right), \\
& \mathrm{E}\left[\operatorname{tr}\left(\boldsymbol{T}_{a} \boldsymbol{T}_{a} \boldsymbol{T}_{a} \boldsymbol{T}_{b} \boldsymbol{T}_{a} \boldsymbol{T}_{b} \boldsymbol{T}_{b} \boldsymbol{T}_{b}\right)\right]=O\left(a b(a+b) p^{6}\right), \\
& \mathrm{E}\left[\operatorname{tr}\left(\boldsymbol{T}_{a} \boldsymbol{T}_{a} \boldsymbol{T}_{b} \boldsymbol{T}_{b} \boldsymbol{T}_{a} \boldsymbol{T}_{a} \boldsymbol{T}_{b} \boldsymbol{T}_{b}\right)\right]=O\left(a b(a+b) p^{6}\right), \\
& \mathrm{E}\left[\operatorname{tr}\left(\boldsymbol{T}_{a} \boldsymbol{T}_{b} \boldsymbol{T}_{a} \boldsymbol{T}_{b} \boldsymbol{T}_{a} \boldsymbol{T}_{b} \boldsymbol{T}_{a} \boldsymbol{T}_{b}\right)\right]=O\left(a b\left(a^{3}+b^{3}\right) p^{4}\right)
\end{aligned}
$$

as $p \rightarrow \infty$ with (4.2).

(ii) If $\boldsymbol{T}_{a} \sim W_{p}\left(a, \boldsymbol{\Sigma}_{a}\right)$ and $\boldsymbol{T}_{b} \sim W_{p}\left(b, \boldsymbol{\Sigma}_{b}\right)$ are independent, then

$$
\begin{aligned}
& \mathrm{E}\left[\operatorname{tr}\left(\boldsymbol{T}_{a} \boldsymbol{A} \boldsymbol{T}_{a} \boldsymbol{B} \boldsymbol{T}_{a} \boldsymbol{C} \boldsymbol{T}_{a} \boldsymbol{D}\right) \operatorname{tr}\left(\boldsymbol{T}_{b} \boldsymbol{E} \boldsymbol{T}_{b} \boldsymbol{F} \boldsymbol{T}_{b} \boldsymbol{G} \boldsymbol{T}_{b} \boldsymbol{H}\right)\right]=O\left(a b p^{8}\right) \\
& \mathrm{E}\left[\operatorname{tr}\left(\boldsymbol{T}_{a} \boldsymbol{A} \boldsymbol{T}_{a} \boldsymbol{B} \boldsymbol{T}_{b} \boldsymbol{C} \boldsymbol{T}_{b} \boldsymbol{D}\right) \operatorname{tr}\left(\boldsymbol{T}_{a} \boldsymbol{E} \boldsymbol{T}_{a} \boldsymbol{F} \boldsymbol{T}_{b} \boldsymbol{G} \boldsymbol{T}_{b} \boldsymbol{H}\right)\right]=O\left(a^{2} b^{2} p^{6}\right) \\
& \mathrm{E}\left[\operatorname{tr}\left(\boldsymbol{T}_{a} \boldsymbol{A} \boldsymbol{T}_{b} \boldsymbol{B} \boldsymbol{T}_{a} \boldsymbol{C} \boldsymbol{T}_{b} \boldsymbol{D}\right) \operatorname{tr}\left(\boldsymbol{T}_{a} \boldsymbol{E} \boldsymbol{T}_{b} \boldsymbol{F} \boldsymbol{T}_{a} \boldsymbol{G} \boldsymbol{T}_{b} \boldsymbol{H}\right)\right]=O\left(a^{2} b^{2}\left(a^{2}+b^{2}\right) p^{4}\right)
\end{aligned}
$$

as $p \rightarrow \infty$ with (4.2) for $p \times p$ matrices $\boldsymbol{A}, \boldsymbol{B}, \boldsymbol{C}, \boldsymbol{D}, \boldsymbol{E}, \boldsymbol{F}, \boldsymbol{G}$, and $\boldsymbol{H}$ which satisfy

$$
\begin{aligned}
& \operatorname{tr}\left(\boldsymbol{\Sigma}_{1} \boldsymbol{\Psi}_{1} \boldsymbol{\Sigma}_{2} \boldsymbol{\Psi}_{2} \boldsymbol{\Sigma}_{3} \boldsymbol{\Psi}_{3} \boldsymbol{\Sigma}_{4} \boldsymbol{\Psi}_{4} \boldsymbol{\Sigma}_{5} \boldsymbol{\Psi}_{5} \boldsymbol{\Sigma}_{6} \boldsymbol{\Psi}_{6} \boldsymbol{\Sigma}_{7} \boldsymbol{\Psi}_{7} \boldsymbol{\Sigma}_{8} \boldsymbol{\Psi}_{8}\right)=O(p), \\
& \boldsymbol{\Sigma}_{i}=\boldsymbol{I}_{p}, \boldsymbol{\Sigma}_{a}, \boldsymbol{\Sigma}_{b},(i=1,2,3,4,5,6,7,8), \\
& \boldsymbol{\Psi}_{i}=\boldsymbol{I}_{p}, \boldsymbol{A}, \boldsymbol{B}, \boldsymbol{C}, \boldsymbol{D}, \boldsymbol{E}, \boldsymbol{F}, \boldsymbol{G}, \boldsymbol{H},(i=1,2,3,4,5,6,7,8) .
\end{aligned}
$$

In partilular, when Assumption 4 is additionaly supposed, the conclusions hold for

$$
\boldsymbol{A}, \boldsymbol{B}, \boldsymbol{C}, \boldsymbol{D}, \boldsymbol{E}, \boldsymbol{F}, \boldsymbol{G}, \boldsymbol{H}=\boldsymbol{I}_{p}, \boldsymbol{\Sigma}_{a}, \boldsymbol{\Sigma}_{b} .
$$

Proof. (i) To avoid showing quite long routine calculations, we simply give a sketch of the proof only for

$$
\mathrm{E}\left[\operatorname{tr}\left(\boldsymbol{T}_{a} \boldsymbol{T}_{a} \boldsymbol{T}_{a} \boldsymbol{T}_{a} \boldsymbol{T}_{b} \boldsymbol{T}_{b} \boldsymbol{T}_{b} \boldsymbol{T}_{b}\right)\right]=O\left(a b p^{7}\right) .
$$

We have

$$
\begin{aligned}
& \mathrm{E}\left[\operatorname{tr}\left(\boldsymbol{T}_{a} \boldsymbol{T}_{a} \boldsymbol{T}_{a} \boldsymbol{T}_{a} \boldsymbol{T}_{b} \boldsymbol{T}_{b} \boldsymbol{T}_{b} \boldsymbol{T}_{b}\right)\right] \\
& =\mathrm{E}\left[\sum_{i=1}^{a} \sum_{j=1}^{a} \sum_{k=1}^{a} \sum_{l=1}^{a} \operatorname{tr}\left(\boldsymbol{\Sigma}_{a}^{\frac{1}{2}} \boldsymbol{z}_{i} \boldsymbol{z}_{i}^{\prime} \boldsymbol{\Sigma}_{a} \boldsymbol{z}_{j} \boldsymbol{z}_{j}^{\prime} \boldsymbol{\Sigma}_{a} \boldsymbol{z}_{k} \boldsymbol{z}_{k}^{\prime} \boldsymbol{\Sigma}_{a} \boldsymbol{z}_{l} \boldsymbol{z}_{l}^{\prime} \boldsymbol{\Sigma}_{a}^{\frac{1}{2}} \boldsymbol{T}_{b} \boldsymbol{T}_{b} \boldsymbol{T}_{b} \boldsymbol{T}_{b}\right)\right] \\
& =\mathrm{E}\left[\sum_{i=1}^{a}\left(\boldsymbol{z}_{i}^{\prime} \boldsymbol{\Sigma}_{a} \boldsymbol{z}_{i} \boldsymbol{z}_{i}^{\prime} \boldsymbol{\Sigma}_{a} \boldsymbol{z}_{i} \boldsymbol{z}_{i}^{\prime} \boldsymbol{\Sigma}_{a} \boldsymbol{z}_{i} \boldsymbol{z}_{i}^{\prime} \boldsymbol{\Sigma}_{a}^{\frac{1}{2}} \boldsymbol{T}_{b} \boldsymbol{T}_{b} \boldsymbol{T}_{b} \boldsymbol{T}_{b} \boldsymbol{\Sigma}_{a}^{\frac{1}{2}} \boldsymbol{z}_{i}\right)\right. \\
& +\sum_{i=1}^{a} \sum_{j \neq i}\left(\boldsymbol{z}_{i}^{\prime} \boldsymbol{\Sigma}_{a} \boldsymbol{z}_{i} \boldsymbol{z}_{i}^{\prime} \boldsymbol{\Sigma}_{a} \boldsymbol{z}_{i} \boldsymbol{z}_{i}^{\prime} \boldsymbol{\Sigma}_{a} \boldsymbol{z}_{j} \boldsymbol{z}_{j}^{\prime} \boldsymbol{\Sigma}_{a}^{\frac{1}{2}} \boldsymbol{T}_{b} \boldsymbol{T}_{b} \boldsymbol{T}_{b} \boldsymbol{T}_{b} \boldsymbol{\Sigma}_{a}^{\frac{1}{2}} \boldsymbol{z}_{i}\right)+\sum_{i=1}^{a} \sum_{j \neq i}\left(\boldsymbol{z}_{i}^{\prime} \boldsymbol{\Sigma}_{a} \boldsymbol{z}_{i} \boldsymbol{z}_{i}^{\prime} \boldsymbol{\Sigma}_{a} \boldsymbol{z}_{j} \boldsymbol{z}_{j}^{\prime} \boldsymbol{\Sigma}_{a} \boldsymbol{z}_{i} \boldsymbol{z}_{i}^{\prime} \boldsymbol{\Sigma}_{a}^{\frac{1}{2}} \boldsymbol{T}_{b} \boldsymbol{T}_{b} \boldsymbol{T}_{b} \boldsymbol{T}_{b} \Sigma_{a}^{\frac{1}{2}} \boldsymbol{z}_{i}\right) \\
& +\sum_{i=1}^{a} \sum_{j \neq i}\left(\boldsymbol{z}_{i}^{\prime} \boldsymbol{\Sigma}_{a} \boldsymbol{z}_{j} \boldsymbol{z}_{j}^{\prime} \boldsymbol{\Sigma}_{a} \boldsymbol{z}_{i} \boldsymbol{z}_{i}^{\prime} \boldsymbol{\Sigma}_{a} \boldsymbol{z}_{i} \boldsymbol{z}_{i}^{\prime} \boldsymbol{\Sigma}_{a}^{\frac{1}{2}} \boldsymbol{T}_{b} \boldsymbol{T}_{b} \boldsymbol{T}_{b} \boldsymbol{T}_{b} \boldsymbol{\Sigma}_{a}^{\frac{1}{2}} \boldsymbol{z}_{i}\right)+\sum_{i=1}^{a} \sum_{j \neq i}\left(\boldsymbol{z}_{i}^{\prime} \boldsymbol{\Sigma}_{a}^{\frac{1}{2}} \boldsymbol{T}_{b} \boldsymbol{T}_{b} \boldsymbol{T}_{b} \boldsymbol{T}_{b} \boldsymbol{\Sigma}_{a}^{\frac{1}{2}} \boldsymbol{z}_{j} \boldsymbol{z}_{j}^{\prime} \boldsymbol{\Sigma}_{a} \boldsymbol{z}_{i} \boldsymbol{z}_{i}^{\prime} \boldsymbol{\Sigma}_{a} \boldsymbol{z}_{i} \boldsymbol{z}_{i}^{\prime} \boldsymbol{\Sigma}_{a} \boldsymbol{z}_{i}\right) \\
& +\sum_{i=1}^{a} \sum_{j \neq i}\left(\boldsymbol{z}_{i}^{\prime} \boldsymbol{\Sigma}_{a} \boldsymbol{z}_{i} \boldsymbol{z}_{i}^{\prime} \boldsymbol{\Sigma}_{a} \boldsymbol{z}_{j} \boldsymbol{z}_{j}^{\prime} \boldsymbol{\Sigma}_{a} \boldsymbol{z}_{j} \boldsymbol{z}_{j}^{\prime} \boldsymbol{\Sigma}_{a}^{\frac{1}{2}} \boldsymbol{T}_{b} \boldsymbol{T}_{b} \boldsymbol{T}_{b} \boldsymbol{T}_{b} \boldsymbol{\Sigma}_{a}^{\frac{1}{2}} \boldsymbol{z}_{i}\right)+\sum_{i=1}^{a} \sum_{j \neq i}\left(\boldsymbol{z}_{i}^{\prime} \boldsymbol{\Sigma}_{a} \boldsymbol{z}_{j} \boldsymbol{z}_{j}^{\prime} \boldsymbol{\Sigma}_{a} \boldsymbol{z}_{i} \boldsymbol{z}_{i}^{\prime} \boldsymbol{\Sigma}_{a} \boldsymbol{z}_{j} \boldsymbol{z}_{j}^{\prime} \boldsymbol{\Sigma}_{a}^{\frac{1}{2}} \boldsymbol{T}_{b} \boldsymbol{T}_{b} \boldsymbol{T}_{b} \boldsymbol{T}_{b} \boldsymbol{\Sigma}_{a}^{\frac{1}{2}} \boldsymbol{z}_{i}\right) \\
& +\sum_{i=1}^{a} \sum_{j \neq i}\left(\boldsymbol{z}_{i}^{\prime} \boldsymbol{\Sigma}_{a} \boldsymbol{z}_{j} \boldsymbol{z}_{j}^{\prime} \boldsymbol{\Sigma}_{a} \boldsymbol{z}_{j} \boldsymbol{z}_{j}^{\prime} \boldsymbol{\Sigma}_{a} \boldsymbol{z}_{i} \boldsymbol{z}_{i}^{\prime} \boldsymbol{\Sigma}_{a}^{\frac{1}{2}} \boldsymbol{T}_{b} \boldsymbol{T}_{b} \boldsymbol{T}_{b} \boldsymbol{T}_{b} \boldsymbol{\Sigma}_{a}^{\frac{1}{2}} \boldsymbol{z}_{i}\right) \\
& +\sum_{i=1}^{a} \sum_{j \neq i} \sum_{k \neq i, j}\left(\boldsymbol{z}_{i}^{\prime} \boldsymbol{\Sigma}_{a} \boldsymbol{z}_{i} \boldsymbol{z}_{i}^{\prime} \boldsymbol{\Sigma}_{a} \boldsymbol{z}_{j} \boldsymbol{z}_{j}^{\prime} \boldsymbol{\Sigma}_{a} \boldsymbol{z}_{k} \boldsymbol{z}_{k}^{\prime} \boldsymbol{\Sigma}_{a}^{\frac{1}{2}} \boldsymbol{T}_{b} \boldsymbol{T}_{b} \boldsymbol{T}_{b} \boldsymbol{T}_{b} \boldsymbol{\Sigma}_{a}^{\frac{1}{2}} \boldsymbol{z}_{i}\right)+\sum_{i=1}^{a} \sum_{j \neq i} \sum_{k \neq i, j}\left(\boldsymbol{z}_{i}^{\prime} \boldsymbol{\Sigma}_{a} \boldsymbol{z}_{j} \boldsymbol{z}_{j}^{\prime} \boldsymbol{\Sigma}_{a} \boldsymbol{z}_{i} \boldsymbol{z}_{i}^{\prime} \boldsymbol{\Sigma}_{a} \boldsymbol{z}_{k} \boldsymbol{z}_{k}^{\prime} \boldsymbol{\Sigma}_{a}^{\frac{1}{2}} \boldsymbol{T}_{b} \boldsymbol{T}_{b} \boldsymbol{T}_{b} \boldsymbol{T}_{b} \boldsymbol{\Sigma}_{a}^{\frac{1}{2}} \boldsymbol{z}_{i}\right) \\
& +\sum_{i=1}^{a} \sum_{j \neq i} \sum_{k \neq i, j}\left(\boldsymbol{z}_{i}^{\prime} \boldsymbol{\Sigma}_{a} \boldsymbol{z}_{j} \boldsymbol{z}_{j}^{\prime} \boldsymbol{\Sigma}_{a} \boldsymbol{z}_{k} \boldsymbol{z}_{k}^{\prime} \boldsymbol{\Sigma}_{a} \boldsymbol{z}_{i} \boldsymbol{z}_{i}^{\prime} \boldsymbol{\Sigma}_{a}^{\frac{1}{2}} \boldsymbol{T}_{b} \boldsymbol{T}_{b} \boldsymbol{T}_{b} \boldsymbol{T}_{b} \boldsymbol{\Sigma}_{a}^{\frac{1}{2}} \boldsymbol{z}_{i}\right)+\sum_{i=1}^{a} \sum_{j \neq i} \sum_{k \neq i, j}\left(\boldsymbol{z}_{j}^{\prime} \boldsymbol{\Sigma}_{a} \boldsymbol{z}_{j} \boldsymbol{z}_{j}^{\prime} \boldsymbol{\Sigma}_{a} \boldsymbol{z}_{k} \boldsymbol{z}_{k}^{\prime} \boldsymbol{\Sigma}_{a}^{\frac{1}{2}} \boldsymbol{T}_{b} \boldsymbol{T}_{b} \boldsymbol{T}_{b} \boldsymbol{T}_{b} \boldsymbol{\Sigma}_{a}^{\frac{1}{2}} \boldsymbol{z}_{i} \boldsymbol{z}_{i}^{\prime} \boldsymbol{\Sigma}_{a} \boldsymbol{z}_{j}\right) \\
& +\sum_{i=1}^{a} \sum_{j \neq i} \sum_{k \neq i, j}\left(\boldsymbol{z}_{j}^{\prime} \boldsymbol{\Sigma}_{a} \boldsymbol{z}_{k} \boldsymbol{z}_{k}^{\prime} \boldsymbol{\Sigma}_{a} \boldsymbol{z}_{j} \boldsymbol{z}_{j}^{\prime} \boldsymbol{\Sigma}_{a}^{\frac{1}{2}} \boldsymbol{T}_{b} \boldsymbol{T}_{b} \boldsymbol{T}_{b} \boldsymbol{T}_{b} \boldsymbol{\Sigma}_{a}^{\frac{1}{2}} \boldsymbol{z}_{i} \boldsymbol{z}_{i}^{\prime} \boldsymbol{\Sigma}_{a} \boldsymbol{z}_{j}\right)+\sum_{i=1}^{a} \sum_{j \neq i} \sum_{k \neq i, j}\left(z_{k}^{\prime} \boldsymbol{\Sigma}_{a}^{\frac{1}{2}} \boldsymbol{T}_{b} \boldsymbol{T}_{b} \boldsymbol{T}_{b} \boldsymbol{T}_{b} \boldsymbol{\Sigma}_{a}^{\frac{1}{2}} \boldsymbol{z}_{i} \boldsymbol{z}_{i}^{\prime} \boldsymbol{\Sigma}_{a} \boldsymbol{z}_{j} \boldsymbol{z}_{j}^{\prime} \boldsymbol{\Sigma}_{a} \boldsymbol{z}_{k} \boldsymbol{z}_{k}^{\prime} \boldsymbol{\Sigma}_{a} \boldsymbol{z}_{k}\right)
\end{aligned}
$$




$$
\begin{aligned}
& \left.+\sum_{i=1}^{a} \sum_{j \neq i} \sum_{k \neq i, j} \sum_{l \neq i, j, k}\left(\boldsymbol{z}_{i}^{\prime} \boldsymbol{\Sigma}_{a} \boldsymbol{z}_{j} \boldsymbol{z}_{j}^{\prime} \boldsymbol{\Sigma}_{a} \boldsymbol{z}_{k} \boldsymbol{z}_{k}^{\prime} \boldsymbol{\Sigma}_{a} \boldsymbol{z}_{l} \boldsymbol{z}_{l}^{\prime} \boldsymbol{\Sigma}_{a}^{\frac{1}{2}} \boldsymbol{T}_{b} \boldsymbol{T}_{b} \boldsymbol{T}_{b} \boldsymbol{T}_{b} \boldsymbol{\Sigma}_{a}^{\frac{1}{2}} \boldsymbol{z}_{i}\right)\right] \\
& \sim \mathrm{E}\left[\sum_{i=1}^{a}\left(\operatorname{tr}\left(\boldsymbol{\Sigma}_{a}\right) \operatorname{tr}\left(\boldsymbol{\Sigma}_{a}\right) \operatorname{tr}\left(\boldsymbol{\Sigma}_{a}\right) \operatorname{tr}\left(\boldsymbol{\Sigma}_{a} \boldsymbol{T}_{b} \boldsymbol{T}_{b} \boldsymbol{T}_{b} \boldsymbol{T}_{b}\right)\right)\right. \\
& +\sum_{i=1}^{a} \sum_{j \neq i}\left(\operatorname{tr}\left(\boldsymbol{\Sigma}_{a}\right) \operatorname{tr}\left(\boldsymbol{\Sigma}_{a}\right) \operatorname{tr}\left(\boldsymbol{\Sigma}_{a}^{2} \boldsymbol{T}_{b} \boldsymbol{T}_{b} \boldsymbol{T}_{b} \boldsymbol{T}_{b}\right)\right)+\sum_{i=1}^{a} \sum_{j \neq i}\left(\operatorname{tr}\left(\boldsymbol{\Sigma}_{a}\right) \operatorname{tr}\left(\boldsymbol{\Sigma}_{a}^{2}\right) \operatorname{tr}\left(\boldsymbol{\Sigma}_{a} \boldsymbol{T}_{b} \boldsymbol{T}_{b} \boldsymbol{T}_{b} \boldsymbol{T}_{b}\right)\right) \\
& +\sum_{i=1}^{a} \sum_{j \neq i}\left(\operatorname{tr}\left(\boldsymbol{\Sigma}_{a}^{2}\right) \operatorname{tr}\left(\boldsymbol{\Sigma}_{a}\right) \operatorname{tr}\left(\boldsymbol{\Sigma}_{a} \boldsymbol{T}_{b} \boldsymbol{T}_{b} \boldsymbol{T}_{b} \boldsymbol{T}_{b}\right)\right)+\sum_{i=1}^{a} \sum_{j \neq i}\left(\operatorname{tr}\left(\boldsymbol{\Sigma}_{a}^{2} \boldsymbol{T}_{b} \boldsymbol{T}_{b} \boldsymbol{T}_{b} \boldsymbol{T}_{b}\right) \operatorname{tr}\left(\boldsymbol{\Sigma}_{a}\right) \operatorname{tr}\left(\boldsymbol{\Sigma}_{a}\right)\right) \\
& +\sum_{i=1}^{a} \sum_{j \neq i}\left(\operatorname{tr}\left(\boldsymbol{\Sigma}_{a}\right) \operatorname{tr}\left(\boldsymbol{\Sigma}_{a}^{2} \boldsymbol{T}_{b} \boldsymbol{T}_{b} \boldsymbol{T}_{b} \boldsymbol{T}_{b}\right) \operatorname{tr}\left(\boldsymbol{\Sigma}_{a}\right)\right)+\sum_{i=1}^{a} \sum_{j \neq i}\left(\boldsymbol{z}_{i}^{\prime} \boldsymbol{\Sigma}_{a}\left(2 \boldsymbol{\Sigma}_{a} \boldsymbol{z}_{i} \boldsymbol{z}_{i}^{\prime} \boldsymbol{\Sigma}_{a}+\operatorname{tr}\left(\boldsymbol{\Sigma}_{a} \boldsymbol{z}_{i} \boldsymbol{z}_{i}^{\prime} \boldsymbol{\Sigma}_{a}\right) \boldsymbol{I}_{p}\right) \boldsymbol{\Sigma}_{a}^{\frac{1}{2}} \boldsymbol{T}_{b} \boldsymbol{T}_{b} \boldsymbol{T}_{b} \boldsymbol{T}_{b} \boldsymbol{\Sigma}_{a}^{\frac{1}{2}} \boldsymbol{z}_{i}\right) \\
& +\sum_{i=1}^{a} \sum_{j \neq i}\left(\operatorname{tr}\left(\boldsymbol{\Sigma}_{a}^{2}\right) \operatorname{tr}\left(\boldsymbol{\Sigma}_{a} \boldsymbol{T}_{b} \boldsymbol{T}_{b} \boldsymbol{T}_{b} \boldsymbol{T}_{b}\right) \operatorname{tr}\left(\boldsymbol{\Sigma}_{a}\right)\right) \\
& +\sum_{i=1}^{a} \sum_{j \neq i} \sum_{k \neq i, j}\left(\operatorname{tr}\left(\boldsymbol{\Sigma}_{a}\right) \operatorname{tr}\left(\boldsymbol{\Sigma}_{a}^{3} \boldsymbol{T}_{b} \boldsymbol{T}_{b} \boldsymbol{T}_{b} \boldsymbol{T}_{b}\right)\right)+\sum_{i=1}^{a} \sum_{j \neq i} \sum_{k \neq i, j}\left(\operatorname{tr}\left(\boldsymbol{\Sigma}_{a}^{2}\right) \operatorname{tr}\left(\boldsymbol{\Sigma}_{a}^{2} \boldsymbol{T}_{b} \boldsymbol{T}_{b} \boldsymbol{T}_{b} \boldsymbol{T}_{b}\right)\right) \\
& +\sum_{i=1}^{a} \sum_{j \neq i} \sum_{k \neq i, j}\left(\operatorname{tr}\left(\boldsymbol{\Sigma}_{a}^{3}\right) \operatorname{tr}\left(\boldsymbol{\Sigma}_{a} \boldsymbol{T}_{b} \boldsymbol{T}_{b} \boldsymbol{T}_{b} \boldsymbol{T}_{b}\right)\right)+\sum_{i=1}^{a} \sum_{j \neq i} \sum_{k \neq i, j}\left(\operatorname{tr}\left(\boldsymbol{\Sigma}_{a}\right) \operatorname{tr}\left(\boldsymbol{\Sigma}_{a}^{3} \boldsymbol{T}_{b} \boldsymbol{T}_{b} \boldsymbol{T}_{b} \boldsymbol{T}_{b}\right)\right) \\
& +\sum_{i=1}^{a} \sum_{j \neq i} \sum_{k \neq i, j}\left(\operatorname{tr}\left(\boldsymbol{\Sigma}_{a}^{2}\right) \operatorname{tr}\left(\boldsymbol{\Sigma}_{a}^{2} \boldsymbol{T}_{b} \boldsymbol{T}_{b} \boldsymbol{T}_{b} \boldsymbol{T}_{b}\right)\right)+\sum_{i=1}^{a} \sum_{j \neq i} \sum_{k \neq i, j}\left(\operatorname{tr}\left(\boldsymbol{\Sigma}_{a}^{3} \boldsymbol{T}_{b} \boldsymbol{T}_{b} \boldsymbol{T}_{b} \boldsymbol{T}_{b}\right) \operatorname{tr}\left(\boldsymbol{\Sigma}_{a}\right)\right) \\
& \left.+\sum_{i=1}^{a} \sum_{j \neq i} \sum_{k \neq i, j} \sum_{l \neq i, j, k}\left(\operatorname{tr}\left(\boldsymbol{\Sigma}_{a}^{4} \boldsymbol{T}_{b} \boldsymbol{T}_{b} \boldsymbol{T}_{b} \boldsymbol{T}_{b}\right)\right)\right] \\
& \sim a \operatorname{tr}\left(\boldsymbol{\Sigma}_{a}\right) \operatorname{tr}\left(\boldsymbol{\Sigma}_{a}\right) \operatorname{tr}\left(\boldsymbol{\Sigma}_{a}\right) \mathrm{E}\left[\operatorname{tr}\left(\boldsymbol{\Sigma}_{a} \boldsymbol{T}_{b} \boldsymbol{T}_{b} \boldsymbol{T}_{b} \boldsymbol{T}_{b}\right)\right] \\
& =O\left(a b p^{7}\right) \text {, }
\end{aligned}
$$

where we use the following facts in the above asymptotic evaluation: Let $\boldsymbol{x} \sim N_{p}\left(\mathbf{0}_{p}, \boldsymbol{I}_{p}\right)$ and $\boldsymbol{Q}, \boldsymbol{R}, \boldsymbol{S}, \boldsymbol{T}$ be $p \times p$ matrices, then

$$
\begin{gathered}
E\left[\boldsymbol{x}\left(\boldsymbol{x}^{\prime} \boldsymbol{Q} \boldsymbol{x}\right) \boldsymbol{x}^{\prime}\right]=\boldsymbol{Q}+\boldsymbol{Q}^{\prime}+\operatorname{tr}(\boldsymbol{Q}) \boldsymbol{I}_{p}, \\
E\left[\left(\boldsymbol{x}^{\prime} \boldsymbol{Q} \boldsymbol{x}\right)\left(\boldsymbol{x}^{\prime} \boldsymbol{R} \boldsymbol{x}\right)\right]=\operatorname{tr}(\boldsymbol{Q}) \operatorname{tr}(\boldsymbol{R})+\operatorname{tr}(\boldsymbol{Q} \boldsymbol{R})+\operatorname{tr}\left(\boldsymbol{Q} \boldsymbol{R}^{\prime}\right),
\end{gathered}
$$

$$
\begin{aligned}
& E\left[\left(\boldsymbol{x}^{\prime} \boldsymbol{Q} \boldsymbol{x}\right)\left(\boldsymbol{x}^{\prime} \boldsymbol{R} \boldsymbol{x}\right)\left(\boldsymbol{x}^{\prime} \boldsymbol{S} \boldsymbol{x}\right)\right] \\
= & \operatorname{tr}(\boldsymbol{Q}) \operatorname{tr}(\boldsymbol{R}) \operatorname{tr}(\boldsymbol{S})+\operatorname{tr}(\boldsymbol{Q}) \operatorname{tr}(\boldsymbol{R S})+\operatorname{tr}(\boldsymbol{Q}) \operatorname{tr}\left(\boldsymbol{R} \boldsymbol{S}^{\prime}\right)+\operatorname{tr}(\boldsymbol{R}) \operatorname{tr}(\boldsymbol{Q S})+\operatorname{tr}(\boldsymbol{R}) \operatorname{tr}\left(\boldsymbol{Q} \boldsymbol{S}^{\prime}\right)+\operatorname{tr}(\boldsymbol{S}) \operatorname{tr}(\boldsymbol{Q R})+\operatorname{tr}(\boldsymbol{S}) \operatorname{tr}\left(\boldsymbol{Q} \boldsymbol{R}^{\prime}\right) \\
& +\operatorname{tr}(\boldsymbol{Q} \boldsymbol{R} \boldsymbol{S})+\operatorname{tr}\left(\boldsymbol{Q} \boldsymbol{R} \boldsymbol{S}^{\prime}\right)+\operatorname{tr}\left(\boldsymbol{Q} \boldsymbol{R}^{\prime} \boldsymbol{S}\right)+\operatorname{tr}\left(\boldsymbol{Q} \boldsymbol{R}^{\prime} \boldsymbol{S}^{\prime}\right)+\operatorname{tr}(\boldsymbol{Q S R})+\operatorname{tr}\left(\boldsymbol{Q} \boldsymbol{S} \boldsymbol{R}^{\prime}\right)+\operatorname{tr}\left(\boldsymbol{Q S}^{\prime} \boldsymbol{R}\right)+\operatorname{tr}\left(\boldsymbol{Q S}^{\prime} \boldsymbol{R}^{\prime}\right),
\end{aligned}
$$

and

$$
\begin{aligned}
& E\left[\left(\boldsymbol{x}^{\prime} \boldsymbol{Q} \boldsymbol{x}\right)\left(\boldsymbol{x}^{\prime} \boldsymbol{R} \boldsymbol{x}\right)\left(\boldsymbol{x}^{\prime} \boldsymbol{S} \boldsymbol{x}\right)\left(\boldsymbol{x}^{\prime} \boldsymbol{T} \boldsymbol{x}\right)\right] \\
& =\operatorname{tr}(\boldsymbol{Q}) \operatorname{tr}(\boldsymbol{R}) \operatorname{tr}(\boldsymbol{S}) \operatorname{tr}(\boldsymbol{T}) \\
& +\operatorname{tr}(\boldsymbol{Q}) \operatorname{tr}(\boldsymbol{R}) \operatorname{tr}(\boldsymbol{S} \boldsymbol{T})+\operatorname{tr}(\boldsymbol{Q}) \operatorname{tr}(\boldsymbol{R}) \operatorname{tr}\left(\boldsymbol{S} \boldsymbol{T}^{\prime}\right)+\operatorname{tr}(\boldsymbol{Q}) \operatorname{tr}(\boldsymbol{S}) \operatorname{tr}(\boldsymbol{R} \boldsymbol{T})+\operatorname{tr}(\boldsymbol{Q}) \operatorname{tr}(\boldsymbol{S}) \operatorname{tr}\left(\boldsymbol{R} \boldsymbol{T}^{\prime}\right) \\
& +\operatorname{tr}(\boldsymbol{Q}) \operatorname{tr}(\boldsymbol{T}) \operatorname{tr}(\boldsymbol{R} \boldsymbol{S})+\operatorname{tr}(\boldsymbol{Q}) \operatorname{tr}(\boldsymbol{T}) \operatorname{tr}\left(\boldsymbol{R} \boldsymbol{S}^{\prime}\right)+\operatorname{tr}(\boldsymbol{R}) \operatorname{tr}(\boldsymbol{S}) \operatorname{tr}(\boldsymbol{Q} \boldsymbol{T})+\operatorname{tr}(\boldsymbol{R}) \operatorname{tr}(\boldsymbol{S}) \operatorname{tr}\left(\boldsymbol{Q} \boldsymbol{T}^{\prime}\right) \\
& +\operatorname{tr}(\boldsymbol{R}) \operatorname{tr}(\boldsymbol{T}) \operatorname{tr}(\boldsymbol{Q S})+\operatorname{tr}(\boldsymbol{R}) \operatorname{tr}(\boldsymbol{T}) \operatorname{tr}\left(\boldsymbol{Q} \boldsymbol{S}^{\prime}\right)+\operatorname{tr}(\boldsymbol{S}) \operatorname{tr}(\boldsymbol{T}) \operatorname{tr}(\boldsymbol{Q R})+\operatorname{tr}(\boldsymbol{S}) \operatorname{tr}(\boldsymbol{T}) \operatorname{tr}\left(\boldsymbol{Q} \boldsymbol{R}^{\prime}\right) \\
& +\operatorname{tr}(\boldsymbol{Q R}) \operatorname{tr}(\boldsymbol{S T})+\operatorname{tr}\left(\boldsymbol{Q} \boldsymbol{R}^{\prime}\right) \operatorname{tr}(\boldsymbol{S T})+\operatorname{tr}(\boldsymbol{Q R}) \operatorname{tr}\left(\boldsymbol{S} \boldsymbol{T}^{\prime}\right)+\operatorname{tr}\left(\boldsymbol{Q} \boldsymbol{R}^{\prime}\right) \operatorname{tr}\left(\boldsymbol{S} \boldsymbol{T}^{\prime}\right)+\operatorname{tr}(\boldsymbol{Q S}) \operatorname{tr}(\boldsymbol{R T})+\operatorname{tr}\left(\boldsymbol{Q} \boldsymbol{S}^{\prime}\right) \operatorname{tr}(\boldsymbol{R T}) \\
& +\operatorname{tr}(\boldsymbol{Q S}) \operatorname{tr}\left(\boldsymbol{R} \boldsymbol{T}^{\prime}\right)+\operatorname{tr}\left(\boldsymbol{Q} \boldsymbol{S}^{\prime}\right) \operatorname{tr}\left(\boldsymbol{R} \boldsymbol{T}^{\prime}\right)+\operatorname{tr}(\boldsymbol{Q} \boldsymbol{T}) \operatorname{tr}(\boldsymbol{R} \boldsymbol{S})+\operatorname{tr}\left(\boldsymbol{Q} \boldsymbol{T}^{\prime}\right) \operatorname{tr}(\boldsymbol{R} \boldsymbol{S})+\operatorname{tr}(\boldsymbol{Q} \boldsymbol{T}) \operatorname{tr}\left(\boldsymbol{R} \boldsymbol{S}^{\prime}\right)+\operatorname{tr}\left(\boldsymbol{Q} \boldsymbol{T}^{\prime}\right) \operatorname{tr}\left(\boldsymbol{R} \boldsymbol{S}^{\prime}\right) \\
& +\operatorname{tr}(\boldsymbol{Q}) \operatorname{tr}(\boldsymbol{R} \boldsymbol{S} \boldsymbol{T})+\operatorname{tr}(\boldsymbol{Q}) \operatorname{tr}\left(\boldsymbol{R} \boldsymbol{S}^{\prime} \boldsymbol{T}\right)+\operatorname{tr}(\boldsymbol{Q}) \operatorname{tr}\left(\boldsymbol{R} \boldsymbol{S} \boldsymbol{T}^{\prime}\right)+\operatorname{tr}(\boldsymbol{Q}) \operatorname{tr}\left(\boldsymbol{R} \boldsymbol{S}^{\prime} \boldsymbol{T}^{\prime}\right)+\operatorname{tr}(\boldsymbol{Q}) \operatorname{tr}(\boldsymbol{R} \boldsymbol{T} \boldsymbol{S})+\operatorname{tr}(\boldsymbol{Q}) \operatorname{tr}\left(\boldsymbol{R} \boldsymbol{T}^{\prime} \boldsymbol{S}\right) \\
& +\operatorname{tr}(\boldsymbol{Q}) \operatorname{tr}\left(\boldsymbol{R} \boldsymbol{T} \boldsymbol{S}^{\prime}\right)+\operatorname{tr}(\boldsymbol{Q}) \operatorname{tr}\left(\boldsymbol{R} \boldsymbol{T}^{\prime} \boldsymbol{S}^{\prime}\right)+\operatorname{tr}(\boldsymbol{R}) \operatorname{tr}(\boldsymbol{Q S T})+\operatorname{tr}(\boldsymbol{R}) \operatorname{tr}\left(\boldsymbol{Q} \boldsymbol{S}^{\prime} \boldsymbol{T}\right)+\operatorname{tr}(\boldsymbol{R}) \operatorname{tr}\left(\boldsymbol{Q} \boldsymbol{S} \boldsymbol{T}^{\prime}\right)+\operatorname{tr}(\boldsymbol{R}) \operatorname{tr}\left(\boldsymbol{Q} \boldsymbol{S}^{\prime} \boldsymbol{T}^{\prime}\right) \\
& +\operatorname{tr}(\boldsymbol{R}) \operatorname{tr}(\boldsymbol{Q T} \boldsymbol{S})+\operatorname{tr}(\boldsymbol{R}) \operatorname{tr}\left(\boldsymbol{Q} \boldsymbol{T}^{\prime} \boldsymbol{S}\right)+\operatorname{tr}(\boldsymbol{R}) \operatorname{tr}\left(\boldsymbol{Q} \boldsymbol{T} \boldsymbol{S}^{\prime}\right)+\operatorname{tr}(\boldsymbol{R}) \operatorname{tr}\left(\boldsymbol{Q} \boldsymbol{T}^{\prime} \boldsymbol{S}^{\prime}\right)+\operatorname{tr}(\boldsymbol{S}) \operatorname{tr}(\boldsymbol{Q R T})+\operatorname{tr}(\boldsymbol{S}) \operatorname{tr}\left(\boldsymbol{Q} \boldsymbol{R}^{\prime} \boldsymbol{T}\right) \\
& +\operatorname{tr}(\boldsymbol{S}) \operatorname{tr}\left(\boldsymbol{Q} \boldsymbol{R} \boldsymbol{T}^{\prime}\right)+\operatorname{tr}(\boldsymbol{S}) \operatorname{tr}\left(\boldsymbol{Q} \boldsymbol{R}^{\prime} \boldsymbol{T}^{\prime}\right)+\operatorname{tr}(\boldsymbol{S}) \operatorname{tr}(\boldsymbol{Q} \boldsymbol{T} \boldsymbol{R})+\operatorname{tr}(\boldsymbol{S}) \operatorname{tr}\left(\boldsymbol{Q} \boldsymbol{T}^{\prime} \boldsymbol{R}\right)+\operatorname{tr}(\boldsymbol{S}) \operatorname{tr}\left(\boldsymbol{Q} \boldsymbol{T} \boldsymbol{R}^{\prime}\right)+\operatorname{tr}(\boldsymbol{S}) \operatorname{tr}\left(\boldsymbol{Q} \boldsymbol{T}^{\prime} \boldsymbol{R}^{\prime}\right) \\
& +\operatorname{tr}(\boldsymbol{T}) \operatorname{tr}(\boldsymbol{Q R} \boldsymbol{S})+\operatorname{tr}(\boldsymbol{T}) \operatorname{tr}\left(\boldsymbol{Q} \boldsymbol{R}^{\prime} \boldsymbol{S}\right)+\operatorname{tr}(\boldsymbol{T}) \operatorname{tr}\left(\boldsymbol{Q} \boldsymbol{R} \boldsymbol{S}^{\prime}\right)+\operatorname{tr}(\boldsymbol{T}) \operatorname{tr}\left(\boldsymbol{Q} \boldsymbol{R}^{\prime} \boldsymbol{S}^{\prime}\right)+\operatorname{tr}(\boldsymbol{T}) \operatorname{tr}(\boldsymbol{Q S} \boldsymbol{R})+\operatorname{tr}(\boldsymbol{T}) \operatorname{tr}\left(\boldsymbol{Q} \boldsymbol{S}^{\prime} \boldsymbol{R}\right)
\end{aligned}
$$




$$
\begin{aligned}
& +\operatorname{tr}(\boldsymbol{T}) \operatorname{tr}\left(\boldsymbol{Q S} \boldsymbol{R}^{\prime}\right)+\operatorname{tr}(\boldsymbol{T}) \operatorname{tr}\left(\boldsymbol{Q} \boldsymbol{S}^{\prime} \boldsymbol{R}^{\prime}\right) \\
& +\operatorname{tr}(\boldsymbol{Q R} \boldsymbol{S} \boldsymbol{T})+\operatorname{tr}\left(\boldsymbol{Q} \boldsymbol{R}^{\prime} \boldsymbol{S} \boldsymbol{T}\right)+\operatorname{tr}\left(\boldsymbol{Q} \boldsymbol{R} \boldsymbol{S}^{\prime} \boldsymbol{T}\right)+\operatorname{tr}\left(\boldsymbol{Q} \boldsymbol{R} \boldsymbol{S} \boldsymbol{T}^{\prime}\right)+\operatorname{tr}\left(\boldsymbol{Q} \boldsymbol{R}^{\prime} \boldsymbol{S}^{\prime} \boldsymbol{T}\right)+\operatorname{tr}\left(\boldsymbol{Q} \boldsymbol{R}^{\prime} \boldsymbol{S} \boldsymbol{T}^{\prime}\right)+\operatorname{tr}\left(\boldsymbol{Q} \boldsymbol{R} \boldsymbol{S}^{\prime} \boldsymbol{T}^{\prime}\right)+\operatorname{tr}\left(\boldsymbol{Q} \boldsymbol{R}^{\prime} \boldsymbol{S}^{\prime} \boldsymbol{T}^{\prime}\right) \\
& +\operatorname{tr}(\boldsymbol{Q R T} \boldsymbol{S})+\operatorname{tr}\left(\boldsymbol{Q} \boldsymbol{R}^{\prime} \boldsymbol{T} \boldsymbol{S}\right)+\operatorname{tr}\left(\boldsymbol{Q} \boldsymbol{R} \boldsymbol{T}^{\prime} \boldsymbol{S}\right)+\operatorname{tr}\left(\boldsymbol{Q R T} \boldsymbol{S}^{\prime}\right)+\operatorname{tr}\left(\boldsymbol{Q} \boldsymbol{R}^{\prime} \boldsymbol{T}^{\prime} \boldsymbol{S}\right)+\operatorname{tr}\left(\boldsymbol{Q} \boldsymbol{R}^{\prime} \boldsymbol{T} \boldsymbol{S}^{\prime}\right)+\operatorname{tr}\left(\boldsymbol{Q} \boldsymbol{R} \boldsymbol{T}^{\prime} \boldsymbol{S}^{\prime}\right)+\operatorname{tr}\left(\boldsymbol{Q} \boldsymbol{R}^{\prime} \boldsymbol{T}^{\prime} \boldsymbol{S}^{\prime}\right) \\
& +\operatorname{tr}(\boldsymbol{Q S} \boldsymbol{R} \boldsymbol{T})+\operatorname{tr}\left(\boldsymbol{Q} \boldsymbol{S}^{\prime} \boldsymbol{R}\right)+\operatorname{tr}\left(\boldsymbol{Q S} \boldsymbol{R}^{\prime} \boldsymbol{T}\right)+\operatorname{tr}\left(\boldsymbol{Q S} \boldsymbol{R} \boldsymbol{T}^{\prime}\right)+\operatorname{tr}\left(\boldsymbol{Q} \boldsymbol{S}^{\prime} \boldsymbol{R}^{\prime} \boldsymbol{T}\right)+\operatorname{tr}\left(\boldsymbol{Q} \boldsymbol{S}^{\prime} \boldsymbol{R} \boldsymbol{T}^{\prime}\right)+\operatorname{tr}\left(\boldsymbol{Q} \boldsymbol{S} \boldsymbol{R}^{\prime} \boldsymbol{T}^{\prime}\right)+\operatorname{tr}\left(\boldsymbol{Q} \boldsymbol{S}^{\prime} \boldsymbol{R}^{\prime} \boldsymbol{T}^{\prime}\right) \\
& +\operatorname{tr}(\boldsymbol{Q S T R})+\operatorname{tr}\left(\boldsymbol{Q} \boldsymbol{S}^{\prime} \boldsymbol{T} \boldsymbol{R}\right)+\operatorname{tr}\left(\boldsymbol{Q S} \boldsymbol{T}^{\prime} \boldsymbol{R}\right)+\operatorname{tr}\left(\boldsymbol{Q S T} \boldsymbol{R}^{\prime}\right)+\operatorname{tr}\left(\boldsymbol{Q} \boldsymbol{S}^{\prime} \boldsymbol{T}^{\prime} \boldsymbol{R}\right)+\operatorname{tr}\left(\boldsymbol{Q} \boldsymbol{S}^{\prime} \boldsymbol{T} \boldsymbol{R}^{\prime}\right)+\operatorname{tr}\left(\boldsymbol{Q} \boldsymbol{S} \boldsymbol{T}^{\prime} \boldsymbol{R}^{\prime}\right)+\operatorname{tr}\left(\boldsymbol{Q} \boldsymbol{S}^{\prime} \boldsymbol{T}^{\prime} \boldsymbol{R}^{\prime}\right) \\
& +\operatorname{tr}(\boldsymbol{Q} \boldsymbol{T} \boldsymbol{R})+\operatorname{tr}\left(\boldsymbol{Q} \boldsymbol{T}^{\prime} \boldsymbol{R}\right)+\operatorname{tr}\left(\boldsymbol{Q} \boldsymbol{T} \boldsymbol{R}^{\prime} \boldsymbol{S}\right)+\operatorname{tr}\left(\boldsymbol{Q} \boldsymbol{T} \boldsymbol{R} \boldsymbol{S}^{\prime}\right)+\operatorname{tr}\left(\boldsymbol{Q} \boldsymbol{T}^{\prime} \boldsymbol{R}^{\prime} \boldsymbol{S}\right)+\operatorname{tr}\left(\boldsymbol{Q} \boldsymbol{T}^{\prime} \boldsymbol{R} \boldsymbol{S}^{\prime}\right)+\operatorname{tr}\left(\boldsymbol{Q} \boldsymbol{T} \boldsymbol{R}^{\prime} \boldsymbol{S}^{\prime}\right)+\operatorname{tr}\left(\boldsymbol{Q} \boldsymbol{T}^{\prime} \boldsymbol{R}^{\prime} \boldsymbol{S}^{\prime}\right) \\
& +\operatorname{tr}(\boldsymbol{Q T S R})+\operatorname{tr}\left(\boldsymbol{Q} \boldsymbol{T}^{\prime} \boldsymbol{S R}\right)+\operatorname{tr}\left(\boldsymbol{Q} \boldsymbol{T} \boldsymbol{S}^{\prime} \boldsymbol{R}\right)+\operatorname{tr}\left(\boldsymbol{Q} \boldsymbol{T} \boldsymbol{S} \boldsymbol{R}^{\prime}\right)+\operatorname{tr}\left(\boldsymbol{Q} \boldsymbol{T}^{\prime} \boldsymbol{S}^{\prime} \boldsymbol{R}\right)+\operatorname{tr}\left(\boldsymbol{Q} \boldsymbol{T}^{\prime} \boldsymbol{S} \boldsymbol{R}^{\prime}\right)+\operatorname{tr}\left(\boldsymbol{Q} \boldsymbol{T} \boldsymbol{S}^{\prime} \boldsymbol{R}^{\prime}\right)+\operatorname{tr}\left(\boldsymbol{Q} \boldsymbol{T}^{\prime} \boldsymbol{S}^{\prime} \boldsymbol{R}^{\prime}\right) .
\end{aligned}
$$

We can prove the rest of the assertions in similar ways, so we omit their details. (ii) Under the assumptions, the assetions of (ii) can be proven by similar arguments to the proof of (i). This completes the proof.

\section{$5 \quad$ Proofs for Section 2}

\subsection{Proof of Proposition 2.1}

The expectation of $\left\{\operatorname{tr}\left(\boldsymbol{T}_{a} \boldsymbol{T}_{b} \boldsymbol{T}_{c} \boldsymbol{T}_{d}\right)^{2}\right\}$ can be calculated as follows:

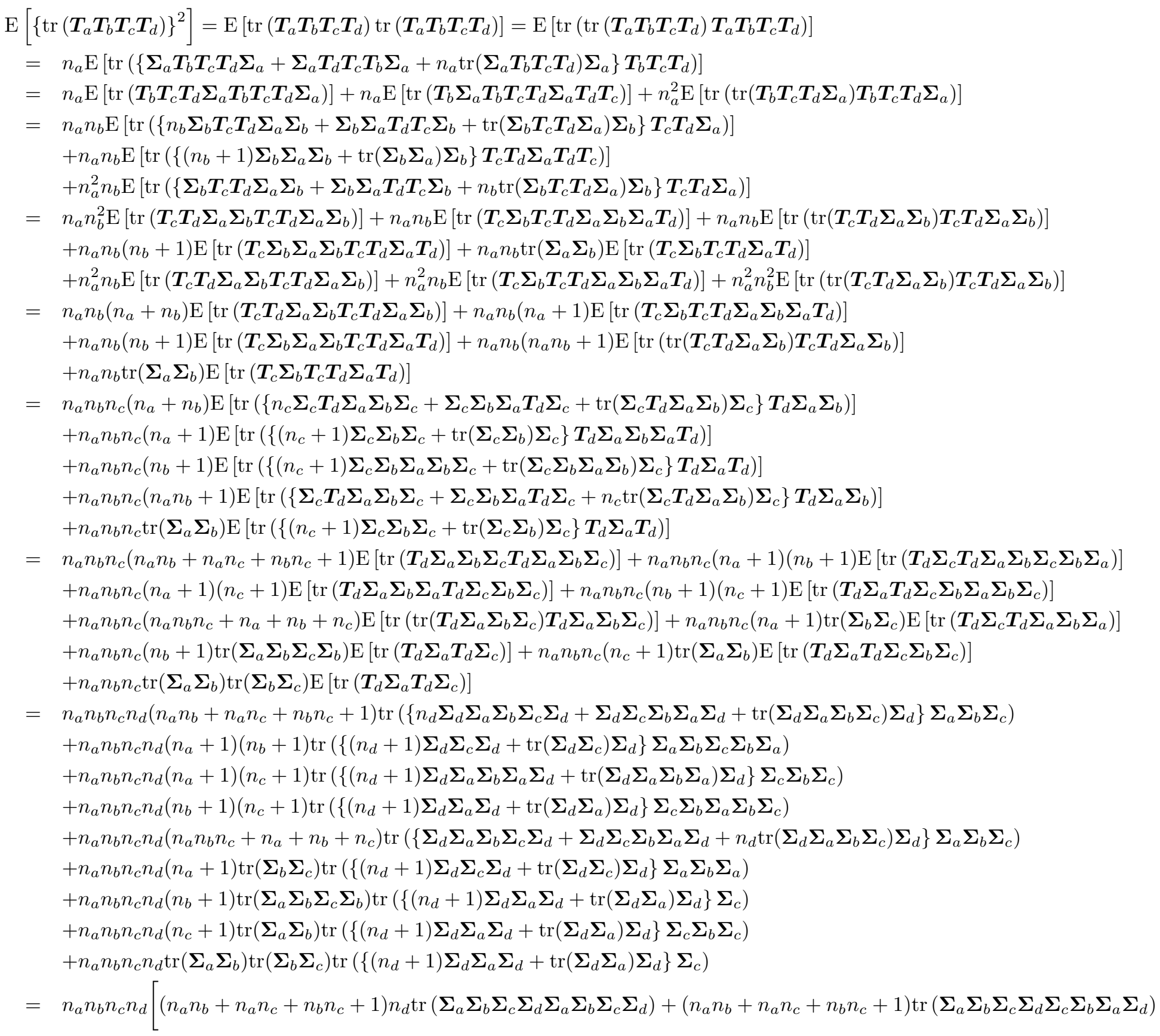




$$
\begin{aligned}
& +\left(n_{a} n_{b}+n_{a} n_{c}+n_{b} n_{c}+1\right)\left\{\operatorname{tr}\left(\boldsymbol{\Sigma}_{a} \boldsymbol{\Sigma}_{b} \boldsymbol{\Sigma}_{c} \boldsymbol{\Sigma}_{d}\right)\right\}^{2} \\
& +\left(n_{a}+1\right)\left(n_{b}+1\right)\left(n_{d}+1\right) \operatorname{tr}\left(\boldsymbol{\Sigma}_{a} \boldsymbol{\Sigma}_{b} \boldsymbol{\Sigma}_{c} \boldsymbol{\Sigma}_{b} \boldsymbol{\Sigma}_{a} \boldsymbol{\Sigma}_{d} \boldsymbol{\Sigma}_{c} \boldsymbol{\Sigma}_{d}\right)+\left(n_{a}+1\right)\left(n_{b}+1\right) \operatorname{tr}\left(\boldsymbol{\Sigma}_{c} \boldsymbol{\Sigma}_{d}\right) \operatorname{tr}\left(\boldsymbol{\Sigma}_{a} \boldsymbol{\Sigma}_{b} \boldsymbol{\Sigma}_{c} \boldsymbol{\Sigma}_{b} \boldsymbol{\Sigma}_{a} \boldsymbol{\Sigma}_{d}\right) \\
& +\left(n_{a}+1\right)\left(n_{c}+1\right)\left(n_{d}+1\right) \operatorname{tr}\left(\boldsymbol{\Sigma}_{a} \boldsymbol{\Sigma}_{b} \boldsymbol{\Sigma}_{a} \boldsymbol{\Sigma}_{d} \boldsymbol{\Sigma}_{c} \boldsymbol{\Sigma}_{b} \boldsymbol{\Sigma}_{c} \boldsymbol{\Sigma}_{d}\right)+\left(n_{a}+1\right)\left(n_{c}+1\right) \operatorname{tr}\left(\boldsymbol{\Sigma}_{a} \boldsymbol{\Sigma}_{b} \boldsymbol{\Sigma}_{a} \boldsymbol{\Sigma}_{d}\right) \operatorname{tr}\left(\boldsymbol{\Sigma}_{b} \boldsymbol{\Sigma}_{c} \boldsymbol{\Sigma}_{d} \boldsymbol{\Sigma}_{c}\right) \\
& +\left(n_{b}+1\right)\left(n_{c}+1\right)\left(n_{d}+1\right) \operatorname{tr}\left(\boldsymbol{\Sigma}_{a} \boldsymbol{\Sigma}_{b} \boldsymbol{\Sigma}_{c} \boldsymbol{\Sigma}_{d} \boldsymbol{\Sigma}_{a} \boldsymbol{\Sigma}_{d} \boldsymbol{\Sigma}_{c} \boldsymbol{\Sigma}_{b}\right)+\left(n_{b}+1\right)\left(n_{c}+1\right) \operatorname{tr}\left(\boldsymbol{\Sigma}_{a} \boldsymbol{\Sigma}_{d}\right) \operatorname{tr}\left(\boldsymbol{\Sigma}_{a} \boldsymbol{\Sigma}_{b} \boldsymbol{\Sigma}_{c} \boldsymbol{\Sigma}_{d} \boldsymbol{\Sigma}_{c} \boldsymbol{\Sigma}_{b}\right) \\
& +\left(n_{a} n_{b} n_{c}+n_{a}+n_{b}+n_{c}\right) \operatorname{tr}\left(\boldsymbol{\Sigma}_{a} \boldsymbol{\Sigma}_{b} \boldsymbol{\Sigma}_{c} \boldsymbol{\Sigma}_{d} \boldsymbol{\Sigma}_{a} \boldsymbol{\Sigma}_{b} \boldsymbol{\Sigma}_{c} \boldsymbol{\Sigma}_{d}\right)+\left(n_{a} n_{b} n_{c}+n_{a}+n_{b}+n_{c}\right) \operatorname{tr}\left(\boldsymbol{\Sigma}_{a} \boldsymbol{\Sigma}_{b} \boldsymbol{\Sigma}_{c} \boldsymbol{\Sigma}_{d} \boldsymbol{\Sigma}_{c} \boldsymbol{\Sigma}_{b} \boldsymbol{\Sigma}_{a} \boldsymbol{\Sigma}_{d}\right) \\
& +\left(n_{a} n_{b} n_{c}+n_{a}+n_{b}+n_{c}\right) n_{d}\left\{\operatorname{tr}\left(\boldsymbol{\Sigma}_{a} \boldsymbol{\Sigma}_{b} \boldsymbol{\Sigma}_{c} \boldsymbol{\Sigma}_{d}\right)\right\}^{2} \\
& +\left(n_{a}+1\right)\left(n_{d}+1\right) \operatorname{tr}\left(\boldsymbol{\Sigma}_{b} \boldsymbol{\Sigma}_{c}\right) \operatorname{tr}\left(\boldsymbol{\Sigma}_{a} \boldsymbol{\Sigma}_{b} \boldsymbol{\Sigma}_{a} \boldsymbol{\Sigma}_{d} \boldsymbol{\Sigma}_{c} \boldsymbol{\Sigma}_{d}\right)+\left(n_{a}+1\right) \operatorname{tr}\left(\boldsymbol{\Sigma}_{b} \boldsymbol{\Sigma}_{c}\right) \operatorname{tr}\left(\boldsymbol{\Sigma}_{c} \boldsymbol{\Sigma}_{d}\right) \operatorname{tr}\left(\boldsymbol{\Sigma}_{a} \boldsymbol{\Sigma}_{b} \boldsymbol{\Sigma}_{a} \boldsymbol{\Sigma}_{d}\right) \\
& +\left(n_{b}+1\right)\left(n_{d}+1\right) \operatorname{tr}\left(\boldsymbol{\Sigma}_{a} \boldsymbol{\Sigma}_{b} \boldsymbol{\Sigma}_{c} \boldsymbol{\Sigma}_{b}\right) \operatorname{tr}\left(\boldsymbol{\Sigma}_{a} \boldsymbol{\Sigma}_{d} \boldsymbol{\Sigma}_{c} \boldsymbol{\Sigma}_{d}\right)+\left(n_{b}+1\right) \operatorname{tr}\left(\boldsymbol{\Sigma}_{a} \boldsymbol{\Sigma}_{d}\right) \operatorname{tr}\left(\boldsymbol{\Sigma}_{c} \boldsymbol{\Sigma}_{d}\right) \operatorname{tr}\left(\boldsymbol{\Sigma}_{a} \boldsymbol{\Sigma}_{b} \boldsymbol{\Sigma}_{c} \boldsymbol{\Sigma}_{b}\right) \\
& +\left(n_{c}+1\right)\left(n_{d}+1\right) \operatorname{tr}\left(\boldsymbol{\Sigma}_{a} \boldsymbol{\Sigma}_{b}\right) \operatorname{tr}\left(\boldsymbol{\Sigma}_{a} \boldsymbol{\Sigma}_{d} \boldsymbol{\Sigma}_{c} \boldsymbol{\Sigma}_{b} \boldsymbol{\Sigma}_{c} \boldsymbol{\Sigma}_{d}\right)+\left(n_{c}+1\right) \operatorname{tr}\left(\boldsymbol{\Sigma}_{a} \boldsymbol{\Sigma}_{b}\right) \operatorname{tr}\left(\boldsymbol{\Sigma}_{a} \boldsymbol{\Sigma}_{d}\right) \operatorname{tr}\left(\boldsymbol{\Sigma}_{b} \boldsymbol{\Sigma}_{c} \boldsymbol{\Sigma}_{d} \boldsymbol{\Sigma}_{c}\right) \\
& \left.+\left(n_{d}+1\right) \operatorname{tr}\left(\boldsymbol{\Sigma}_{a} \boldsymbol{\Sigma}_{b}\right) \operatorname{tr}\left(\boldsymbol{\Sigma}_{b} \boldsymbol{\Sigma}_{c}\right) \operatorname{tr}\left(\boldsymbol{\Sigma}_{a} \boldsymbol{\Sigma}_{d} \boldsymbol{\Sigma}_{c} \boldsymbol{\Sigma}_{d}\right)+\operatorname{tr}\left(\boldsymbol{\Sigma}_{a} \boldsymbol{\Sigma}_{b}\right) \operatorname{tr}\left(\boldsymbol{\Sigma}_{a} \boldsymbol{\Sigma}_{d}\right) \operatorname{tr}\left(\boldsymbol{\Sigma}_{b} \boldsymbol{\Sigma}_{c}\right) \operatorname{tr}\left(\boldsymbol{\Sigma}_{c} \boldsymbol{\Sigma}_{d}\right)\right] \\
& =n_{a} n_{b} n_{c} n_{d}\left[\left(n_{a} n_{b} n_{c} n_{d}+n_{a} n_{b}+n_{a} n_{c}+n_{a} n_{d}+n_{b} n_{c}+n_{b} n_{d}+n_{c} n_{d}+1\right)\left\{\operatorname{tr}\left(\boldsymbol{\Sigma}_{a} \boldsymbol{\Sigma}_{b} \boldsymbol{\Sigma}_{c} \boldsymbol{\Sigma}_{d}\right)\right\}^{2}\right. \\
& +\left(n_{a} n_{b} n_{c}+n_{a} n_{b} n_{d}+n_{a} n_{c} n_{d}+n_{b} n_{c} n_{d}+n_{a}+n_{b}+n_{c}+n_{d}\right) \operatorname{tr}\left(\boldsymbol{\Sigma}_{a} \boldsymbol{\Sigma}_{b} \boldsymbol{\Sigma}_{c} \boldsymbol{\Sigma}_{d} \boldsymbol{\Sigma}_{a} \boldsymbol{\Sigma}_{b} \boldsymbol{\Sigma}_{c} \boldsymbol{\Sigma}_{d}\right) \\
& +\left(n_{a}+1\right)\left(n_{b}+1\right)\left(n_{c}+1\right) \operatorname{tr}\left(\boldsymbol{\Sigma}_{a} \boldsymbol{\Sigma}_{b} \boldsymbol{\Sigma}_{c} \boldsymbol{\Sigma}_{d} \boldsymbol{\Sigma}_{c} \boldsymbol{\Sigma}_{b} \boldsymbol{\Sigma}_{a} \boldsymbol{\Sigma}_{d}\right)+\left(n_{a}+1\right)\left(n_{b}+1\right)\left(n_{d}+1\right) \operatorname{tr}\left(\boldsymbol{\Sigma}_{a} \boldsymbol{\Sigma}_{b} \boldsymbol{\Sigma}_{c} \boldsymbol{\Sigma}_{b} \boldsymbol{\Sigma}_{a} \boldsymbol{\Sigma}_{d} \boldsymbol{\Sigma}_{c} \boldsymbol{\Sigma}_{d}\right) \\
& +\left(n_{a}+1\right)\left(n_{c}+1\right)\left(n_{d}+1\right) \operatorname{tr}\left(\boldsymbol{\Sigma}_{a} \boldsymbol{\Sigma}_{b} \boldsymbol{\Sigma}_{a} \boldsymbol{\Sigma}_{d} \boldsymbol{\Sigma}_{c} \boldsymbol{\Sigma}_{b} \boldsymbol{\Sigma}_{c} \boldsymbol{\Sigma}_{d}\right)+\left(n_{b}+1\right)\left(n_{c}+1\right)\left(n_{d}+1\right) \operatorname{tr}\left(\boldsymbol{\Sigma}_{a} \boldsymbol{\Sigma}_{b} \boldsymbol{\Sigma}_{c} \boldsymbol{\Sigma}_{d} \boldsymbol{\Sigma}_{a} \boldsymbol{\Sigma}_{d} \boldsymbol{\Sigma}_{c} \boldsymbol{\Sigma}_{b}\right) \\
& +\left(n_{a}+1\right)\left(n_{b}+1\right) \operatorname{tr}\left(\boldsymbol{\Sigma}_{c} \boldsymbol{\Sigma}_{d}\right) \operatorname{tr}\left(\boldsymbol{\Sigma}_{a} \boldsymbol{\Sigma}_{b} \boldsymbol{\Sigma}_{c} \boldsymbol{\Sigma}_{b} \boldsymbol{\Sigma}_{a} \boldsymbol{\Sigma}_{d}\right)+\left(n_{a}+1\right)\left(n_{c}+1\right) \operatorname{tr}\left(\boldsymbol{\Sigma}_{a} \boldsymbol{\Sigma}_{b} \boldsymbol{\Sigma}_{a} \boldsymbol{\Sigma}_{d}\right) \operatorname{tr}\left(\boldsymbol{\Sigma}_{b} \boldsymbol{\Sigma}_{c} \boldsymbol{\Sigma}_{d} \boldsymbol{\Sigma}_{c}\right) \\
& +\left(n_{a}+1\right)\left(n_{d}+1\right) \operatorname{tr}\left(\boldsymbol{\Sigma}_{b} \boldsymbol{\Sigma}_{c}\right) \operatorname{tr}\left(\boldsymbol{\Sigma}_{a} \boldsymbol{\Sigma}_{b} \boldsymbol{\Sigma}_{a} \boldsymbol{\Sigma}_{d} \boldsymbol{\Sigma}_{c} \boldsymbol{\Sigma}_{d}\right)+\left(n_{b}+1\right)\left(n_{c}+1\right) \operatorname{tr}\left(\boldsymbol{\Sigma}_{a} \boldsymbol{\Sigma}_{d}\right) \operatorname{tr}\left(\boldsymbol{\Sigma}_{a} \boldsymbol{\Sigma}_{b} \boldsymbol{\Sigma}_{c} \boldsymbol{\Sigma}_{d} \boldsymbol{\Sigma}_{c} \boldsymbol{\Sigma}_{b}\right) \\
& +\left(n_{b}+1\right)\left(n_{d}+1\right) \operatorname{tr}\left(\boldsymbol{\Sigma}_{a} \boldsymbol{\Sigma}_{b} \boldsymbol{\Sigma}_{c} \boldsymbol{\Sigma}_{b}\right) \operatorname{tr}\left(\boldsymbol{\Sigma}_{a} \boldsymbol{\Sigma}_{d} \boldsymbol{\Sigma}_{c} \boldsymbol{\Sigma}_{d}\right)+\left(n_{c}+1\right)\left(n_{d}+1\right) \operatorname{tr}\left(\boldsymbol{\Sigma}_{a} \boldsymbol{\Sigma}_{b}\right) \operatorname{tr}\left(\boldsymbol{\Sigma}_{a} \boldsymbol{\Sigma}_{d} \boldsymbol{\Sigma}_{c} \boldsymbol{\Sigma}_{b} \boldsymbol{\Sigma}_{c} \boldsymbol{\Sigma}_{d}\right) \\
& +\left(n_{a}+1\right) \operatorname{tr}\left(\boldsymbol{\Sigma}_{b} \boldsymbol{\Sigma}_{c}\right) \operatorname{tr}\left(\boldsymbol{\Sigma}_{c} \boldsymbol{\Sigma}_{d}\right) \operatorname{tr}\left(\boldsymbol{\Sigma}_{a} \boldsymbol{\Sigma}_{b} \boldsymbol{\Sigma}_{a} \boldsymbol{\Sigma}_{d}\right)+\left(n_{b}+1\right) \operatorname{tr}\left(\boldsymbol{\Sigma}_{a} \boldsymbol{\Sigma}_{d}\right) \operatorname{tr}\left(\boldsymbol{\Sigma}_{c} \boldsymbol{\Sigma}_{d}\right) \operatorname{tr}\left(\boldsymbol{\Sigma}_{a} \boldsymbol{\Sigma}_{b} \boldsymbol{\Sigma}_{c} \boldsymbol{\Sigma}_{b}\right) \\
& +\left(n_{c}+1\right) \operatorname{tr}\left(\boldsymbol{\Sigma}_{a} \boldsymbol{\Sigma}_{b}\right) \operatorname{tr}\left(\boldsymbol{\Sigma}_{a} \boldsymbol{\Sigma}_{d}\right) \operatorname{tr}\left(\boldsymbol{\Sigma}_{b} \boldsymbol{\Sigma}_{c} \boldsymbol{\Sigma}_{d} \boldsymbol{\Sigma}_{c}\right)+\left(n_{d}+1\right) \operatorname{tr}\left(\boldsymbol{\Sigma}_{a} \boldsymbol{\Sigma}_{b}\right) \operatorname{tr}\left(\boldsymbol{\Sigma}_{b} \boldsymbol{\Sigma}_{c}\right) \operatorname{tr}\left(\boldsymbol{\Sigma}_{a} \boldsymbol{\Sigma}_{d} \boldsymbol{\Sigma}_{c} \boldsymbol{\Sigma}_{d}\right) \\
& \left.+\operatorname{tr}\left(\boldsymbol{\Sigma}_{a} \boldsymbol{\Sigma}_{b}\right) \operatorname{tr}\left(\boldsymbol{\Sigma}_{a} \boldsymbol{\Sigma}_{d}\right) \operatorname{tr}\left(\boldsymbol{\Sigma}_{b} \boldsymbol{\Sigma}_{c}\right) \operatorname{tr}\left(\boldsymbol{\Sigma}_{c} \boldsymbol{\Sigma}_{d}\right)\right]
\end{aligned}
$$

Hence, the variance of $\operatorname{tr}\left(\boldsymbol{T}_{a} \boldsymbol{T}_{b} \boldsymbol{T}_{c} \boldsymbol{T}_{d}\right)$ is

$$
\begin{aligned}
& \mathrm{V}\left[\operatorname{tr}\left(\boldsymbol{T}_{a} \boldsymbol{T}_{b} \boldsymbol{T}_{c} \boldsymbol{T}_{d}\right)\right] \\
& =n_{a} n_{b} n_{c} n_{d}\left[\left(n_{a} n_{b}+n_{a} n_{c}+n_{a} n_{d}+n_{b} n_{c}+n_{b} n_{d}+n_{c} n_{d}+1\right)\left\{\operatorname{tr}\left(\boldsymbol{\Sigma}_{a} \boldsymbol{\Sigma}_{b} \boldsymbol{\Sigma}_{c} \boldsymbol{\Sigma}_{d}\right)\right\}^{2}\right. \\
& +\left(n_{a} n_{b} n_{c}+n_{a} n_{b} n_{d}+n_{a} n_{c} n_{d}+n_{b} n_{c} n_{d}+n_{a}+n_{b}+n_{c}+n_{d}\right) \operatorname{tr}\left(\boldsymbol{\Sigma}_{a} \boldsymbol{\Sigma}_{b} \boldsymbol{\Sigma}_{c} \boldsymbol{\Sigma}_{d} \boldsymbol{\Sigma}_{a} \boldsymbol{\Sigma}_{b} \boldsymbol{\Sigma}_{c} \boldsymbol{\Sigma}_{d}\right) \\
& +\left(n_{a}+1\right)\left(n_{b}+1\right)\left(n_{c}+1\right) \operatorname{tr}\left(\boldsymbol{\Sigma}_{a} \boldsymbol{\Sigma}_{b} \boldsymbol{\Sigma}_{c} \boldsymbol{\Sigma}_{d} \boldsymbol{\Sigma}_{c} \boldsymbol{\Sigma}_{b} \boldsymbol{\Sigma}_{a} \boldsymbol{\Sigma}_{d}\right)+\left(n_{a}+1\right)\left(n_{b}+1\right)\left(n_{d}+1\right) \operatorname{tr}\left(\boldsymbol{\Sigma}_{a} \boldsymbol{\Sigma}_{b} \boldsymbol{\Sigma}_{c} \boldsymbol{\Sigma}_{b} \boldsymbol{\Sigma}_{a} \boldsymbol{\Sigma}_{d} \boldsymbol{\Sigma}_{c} \boldsymbol{\Sigma}_{d}\right) \\
& +\left(n_{a}+1\right)\left(n_{c}+1\right)\left(n_{d}+1\right) \operatorname{tr}\left(\boldsymbol{\Sigma}_{a} \boldsymbol{\Sigma}_{b} \boldsymbol{\Sigma}_{a} \boldsymbol{\Sigma}_{d} \boldsymbol{\Sigma}_{c} \boldsymbol{\Sigma}_{b} \boldsymbol{\Sigma}_{c} \boldsymbol{\Sigma}_{d}\right)+\left(n_{b}+1\right)\left(n_{c}+1\right)\left(n_{d}+1\right) \operatorname{tr}\left(\boldsymbol{\Sigma}_{a} \boldsymbol{\Sigma}_{b} \boldsymbol{\Sigma}_{c} \boldsymbol{\Sigma}_{d} \boldsymbol{\Sigma}_{a} \boldsymbol{\Sigma}_{d} \boldsymbol{\Sigma}_{c} \boldsymbol{\Sigma}_{b}\right) \\
& +\left(n_{a}+1\right)\left(n_{b}+1\right) \operatorname{tr}\left(\boldsymbol{\Sigma}_{c} \boldsymbol{\Sigma}_{d}\right) \operatorname{tr}\left(\boldsymbol{\Sigma}_{a} \boldsymbol{\Sigma}_{b} \boldsymbol{\Sigma}_{c} \boldsymbol{\Sigma}_{b} \boldsymbol{\Sigma}_{a} \boldsymbol{\Sigma}_{d}\right)+\left(n_{a}+1\right)\left(n_{c}+1\right) \operatorname{tr}\left(\boldsymbol{\Sigma}_{a} \boldsymbol{\Sigma}_{b} \boldsymbol{\Sigma}_{a} \boldsymbol{\Sigma}_{d}\right) \operatorname{tr}\left(\boldsymbol{\Sigma}_{b} \boldsymbol{\Sigma}_{c} \boldsymbol{\Sigma}_{d} \boldsymbol{\Sigma}_{c}\right) \\
& +\left(n_{a}+1\right)\left(n_{d}+1\right) \operatorname{tr}\left(\boldsymbol{\Sigma}_{b} \boldsymbol{\Sigma}_{c}\right) \operatorname{tr}\left(\boldsymbol{\Sigma}_{a} \boldsymbol{\Sigma}_{b} \boldsymbol{\Sigma}_{a} \boldsymbol{\Sigma}_{d} \boldsymbol{\Sigma}_{c} \boldsymbol{\Sigma}_{d}\right)+\left(n_{b}+1\right)\left(n_{c}+1\right) \operatorname{tr}\left(\boldsymbol{\Sigma}_{a} \boldsymbol{\Sigma}_{d}\right) \operatorname{tr}\left(\boldsymbol{\Sigma}_{a} \boldsymbol{\Sigma}_{b} \boldsymbol{\Sigma}_{c} \boldsymbol{\Sigma}_{d} \boldsymbol{\Sigma}_{c} \boldsymbol{\Sigma}_{b}\right) \\
& +\left(n_{b}+1\right)\left(n_{d}+1\right) \operatorname{tr}\left(\boldsymbol{\Sigma}_{a} \boldsymbol{\Sigma}_{b} \boldsymbol{\Sigma}_{c} \boldsymbol{\Sigma}_{b}\right) \operatorname{tr}\left(\boldsymbol{\Sigma}_{a} \boldsymbol{\Sigma}_{d} \boldsymbol{\Sigma}_{c} \boldsymbol{\Sigma}_{d}\right)+\left(n_{c}+1\right)\left(n_{d}+1\right) \operatorname{tr}\left(\boldsymbol{\Sigma}_{a} \boldsymbol{\Sigma}_{b}\right) \operatorname{tr}\left(\boldsymbol{\Sigma}_{a} \boldsymbol{\Sigma}_{d} \boldsymbol{\Sigma}_{c} \boldsymbol{\Sigma}_{b} \boldsymbol{\Sigma}_{c} \boldsymbol{\Sigma}_{d}\right) \\
& +\left(n_{a}+1\right) \operatorname{tr}\left(\boldsymbol{\Sigma}_{b} \boldsymbol{\Sigma}_{c}\right) \operatorname{tr}\left(\boldsymbol{\Sigma}_{c} \boldsymbol{\Sigma}_{d}\right) \operatorname{tr}\left(\boldsymbol{\Sigma}_{a} \boldsymbol{\Sigma}_{b} \boldsymbol{\Sigma}_{a} \boldsymbol{\Sigma}_{d}\right)+\left(n_{b}+1\right) \operatorname{tr}\left(\boldsymbol{\Sigma}_{a} \boldsymbol{\Sigma}_{d}\right) \operatorname{tr}\left(\boldsymbol{\Sigma}_{c} \boldsymbol{\Sigma}_{d}\right) \operatorname{tr}\left(\boldsymbol{\Sigma}_{a} \boldsymbol{\Sigma}_{b} \boldsymbol{\Sigma}_{c} \boldsymbol{\Sigma}_{b}\right) \\
& +\left(n_{c}+1\right) \operatorname{tr}\left(\boldsymbol{\Sigma}_{a} \boldsymbol{\Sigma}_{b}\right) \operatorname{tr}\left(\boldsymbol{\Sigma}_{a} \boldsymbol{\Sigma}_{d}\right) \operatorname{tr}\left(\boldsymbol{\Sigma}_{b} \boldsymbol{\Sigma}_{c} \boldsymbol{\Sigma}_{d} \boldsymbol{\Sigma}_{c}\right)+\left(n_{d}+1\right) \operatorname{tr}\left(\boldsymbol{\Sigma}_{a} \boldsymbol{\Sigma}_{b}\right) \operatorname{tr}\left(\boldsymbol{\Sigma}_{b} \boldsymbol{\Sigma}_{c}\right) \operatorname{tr}\left(\boldsymbol{\Sigma}_{a} \boldsymbol{\Sigma}_{d} \boldsymbol{\Sigma}_{c} \boldsymbol{\Sigma}_{d}\right) \\
& \left.+\operatorname{tr}\left(\boldsymbol{\Sigma}_{a} \boldsymbol{\Sigma}_{b}\right) \operatorname{tr}\left(\boldsymbol{\Sigma}_{a} \boldsymbol{\Sigma}_{d}\right) \operatorname{tr}\left(\boldsymbol{\Sigma}_{b} \boldsymbol{\Sigma}_{c}\right) \operatorname{tr}\left(\boldsymbol{\Sigma}_{c} \boldsymbol{\Sigma}_{d}\right)\right] .
\end{aligned}
$$

Therefore, the conclusion follows from Assumption 1. This completes the proof of Proposition 2.1.

\subsection{Proof of Lemma 2.3}

From the definitions of $\boldsymbol{T}_{a}\left(n_{a}\right), \boldsymbol{T}_{b}\left(n_{b}\right), \boldsymbol{T}_{c}\left(n_{c}\right), \boldsymbol{T}_{d}\left(n_{d}\right)$,

$$
\mathrm{E}_{h}[M]=\mathrm{E}_{h}\left[\frac{1}{r_{p}} \operatorname{tr}\left(\boldsymbol{T}_{a}\left(n_{a}\right) \boldsymbol{T}_{b}\left(n_{b}\right) \boldsymbol{T}_{c}\left(n_{c}\right) \boldsymbol{T}_{d}\left(n_{d}\right)\right)\right]
$$

is given as follows:

- for $h=1, \ldots, n_{a}$, it holds that

$$
\mathrm{E}_{h}[M]=\frac{n_{b} n_{c} n_{d}}{r_{p}} \operatorname{tr}\left(\left\{\boldsymbol{T}_{a}(h)+\left(n_{a}-h\right) \boldsymbol{\Sigma}_{a}\right\} \boldsymbol{\Sigma}_{b} \boldsymbol{\Sigma}_{c} \boldsymbol{\Sigma}_{d}\right)
$$


- for $h=n_{a}+1, \ldots, n_{a}+n_{b}$, it holds that

$$
\mathrm{E}_{h}[M]=\frac{n_{c} n_{d}}{r_{p}} \operatorname{tr}\left(\boldsymbol{T}_{a}\left(n_{a}\right)\left\{\boldsymbol{T}_{b}\left(h-n_{a}\right)+\left(n_{a}+n_{b}-h\right) \boldsymbol{\Sigma}_{b}\right\} \boldsymbol{\Sigma}_{c} \boldsymbol{\Sigma}_{d}\right) ;
$$

- for $h=n_{a}+n_{b}+1, \ldots, n_{a}+n_{b}+n_{c}$, it holds that

$$
\mathrm{E}_{h}[M]=\frac{n_{d}}{r_{p}} \operatorname{tr}\left(\boldsymbol{T}_{a}\left(n_{a}\right) \boldsymbol{T}_{b}\left(n_{b}\right)\left\{\boldsymbol{T}_{c}\left(h-n_{a}-n_{b}\right)+\left(n_{a}+n_{b}+n_{c}-h\right) \boldsymbol{\Sigma}_{c}\right\} \boldsymbol{\Sigma}_{d}\right) ;
$$

- for $h=n_{a}+n_{b}+n_{c}+1, \ldots, n_{a}+n_{b}+n_{c}+n_{d}$, it holds that

$$
\begin{aligned}
\mathrm{E}_{h}[M]= & \frac{1}{r_{p}} \operatorname{tr}\left(\boldsymbol{T}_{a}\left(n_{a}\right) \boldsymbol{T}_{b}\left(n_{b}\right) \boldsymbol{T}_{c}\left(n_{c}\right)\right. \\
& \left.\times\left\{\boldsymbol{T}_{d}\left(h-n_{a}-n_{b}-n_{c}\right)+\left(n_{a}+n_{b}+n_{c}+n_{d}-h\right) \boldsymbol{\Sigma}_{d}\right\}\right) .
\end{aligned}
$$

Hence, $D_{h}=\mathrm{E}_{h}[M]-\mathrm{E}_{h-1}[M]$ is given as follows:

- for $h=1, \ldots, n_{a}$, it holds that

$$
D_{h}=\frac{n_{b} n_{c} n_{d}}{r_{p}} \operatorname{tr}\left(\left(\boldsymbol{x}_{h} \boldsymbol{x}_{h}^{\prime}-\boldsymbol{\Sigma}_{a}\right) \boldsymbol{\Sigma}_{b} \boldsymbol{\Sigma}_{c} \boldsymbol{\Sigma}_{d}\right)
$$

- for $h=n_{a}+1, \ldots, n_{a}+n_{b}$, it holds that

$$
D_{h}=\frac{n_{c} n_{d}}{r_{p}} \operatorname{tr}\left(\boldsymbol{T}_{a}\left(n_{a}\right)\left(\boldsymbol{y}_{h-n_{a}} \boldsymbol{y}_{h-n_{a}}^{\prime}-\boldsymbol{\Sigma}_{b}\right) \boldsymbol{\Sigma}_{c} \boldsymbol{\Sigma}_{d}\right)
$$

- for $h=n_{a}+n_{b}+1, \ldots, n_{a}+n_{b}+n_{c}$, it holds that

$$
D_{h}=\frac{n_{d}}{r_{p}} \operatorname{tr}\left(\boldsymbol{T}_{a}\left(n_{a}\right) \boldsymbol{T}_{b}\left(n_{b}\right)\left(\boldsymbol{z}_{h-n_{a}-n_{b}} \boldsymbol{z}_{h-n_{a}-n_{b}}^{\prime}-\boldsymbol{\Sigma}_{c}\right) \boldsymbol{\Sigma}_{d}\right)
$$

- for $h=n_{a}+n_{b}+n_{c}+1, \ldots, n_{a}+n_{b}+n_{c}+n_{d}$, it holds that

$$
D_{h}=\frac{1}{r_{p}} \operatorname{tr}\left(\boldsymbol{T}_{a}\left(n_{a}\right) \boldsymbol{T}_{b}\left(n_{b}\right) \boldsymbol{T}_{c}\left(n_{c}\right)\left(\boldsymbol{w}_{h-n_{a}-n_{b}-n_{c}} \boldsymbol{w}_{h-n_{a}-n_{b}-n_{c}}^{\prime}-\boldsymbol{\Sigma}_{d}\right)\right) .
$$

Recalling that $\sigma_{h}^{2}=\mathrm{E}_{h-1}\left[D_{h}^{2}\right]$, by using Lemma $4.2, \sigma_{h}^{2}$ is given as follow:

- for $h=1, \ldots, n_{a}$, it holds that

$$
\begin{aligned}
\sigma_{h}^{2}= & \mathrm{E}_{h-1}\left[\left\{\frac{n_{b} n_{c} n_{d}}{r_{p}} \operatorname{tr}\left(\left(\boldsymbol{x}_{h} \boldsymbol{x}_{h}^{\prime}-\boldsymbol{\Sigma}_{a}\right) \boldsymbol{\Sigma}_{b} \boldsymbol{\Sigma}_{c} \boldsymbol{\Sigma}_{d}\right)\right\}^{2}\right] \\
= & \frac{n_{b}^{2} n_{c}^{2} n_{d}^{2}}{r_{p}^{2}} \mathrm{E}_{h-1}\left[\left\{\boldsymbol{x}_{h}^{\prime} \boldsymbol{\Sigma}_{a}^{-\frac{1}{2}} \boldsymbol{\Sigma}_{a}^{\frac{1}{2}} \boldsymbol{\Sigma}_{b} \boldsymbol{\Sigma}_{c} \boldsymbol{\Sigma}_{d} \boldsymbol{\Sigma}_{a}^{\frac{1}{2}} \boldsymbol{\Sigma}_{a}^{-\frac{1}{2}} \boldsymbol{x}_{h}-\operatorname{tr}\left(\boldsymbol{\Sigma}_{a}^{\frac{1}{2}} \boldsymbol{\Sigma}_{b} \boldsymbol{\Sigma}_{c} \boldsymbol{\Sigma}_{d} \boldsymbol{\Sigma}_{a}^{\frac{1}{2}}\right)\right\}^{2}\right] \\
= & \frac{n_{b}^{2} n_{c}^{2} n_{d}^{2}}{r_{p}^{2}}\left\{\operatorname{tr}\left(\left(\boldsymbol{\Sigma}_{a}^{\frac{1}{2}} \boldsymbol{\Sigma}_{b} \boldsymbol{\Sigma}_{c} \boldsymbol{\Sigma}_{d} \boldsymbol{\Sigma}_{a}^{\frac{1}{2}}\right)\left(\boldsymbol{\Sigma}_{a}^{\frac{1}{2}} \boldsymbol{\Sigma}_{b} \boldsymbol{\Sigma}_{c} \boldsymbol{\Sigma}_{d} \boldsymbol{\Sigma}_{a}^{\frac{1}{2}}\right)\right)\right. \\
& \left.+\operatorname{tr}\left(\left(\boldsymbol{\Sigma}_{a}^{\frac{1}{2}} \boldsymbol{\Sigma}_{b} \boldsymbol{\Sigma}_{c} \boldsymbol{\Sigma}_{d} \boldsymbol{\Sigma}_{a}^{\frac{1}{2}}\right)\left(\boldsymbol{\Sigma}_{a}^{\frac{1}{2}} \boldsymbol{\Sigma}_{d} \boldsymbol{\Sigma}_{c} \boldsymbol{\Sigma}_{b} \boldsymbol{\Sigma}_{a}^{\frac{1}{2}}\right)\right)\right\} \\
= & \frac{n_{b}^{2} n_{c}^{2} n_{d}^{2}}{r_{p}^{2}}\left\{\operatorname{tr}\left(\boldsymbol{\Sigma}_{a} \boldsymbol{\Sigma}_{b} \boldsymbol{\Sigma}_{c} \boldsymbol{\Sigma}_{d} \boldsymbol{\Sigma}_{a} \boldsymbol{\Sigma}_{b} \boldsymbol{\Sigma}_{c} \boldsymbol{\Sigma}_{d}\right)+\operatorname{tr}\left(\boldsymbol{\Sigma}_{a} \boldsymbol{\Sigma}_{b} \boldsymbol{\Sigma}_{c} \boldsymbol{\Sigma}_{d} \boldsymbol{\Sigma}_{a} \boldsymbol{\Sigma}_{d} \boldsymbol{\Sigma}_{c} \boldsymbol{\Sigma}_{b}\right)\right\}
\end{aligned}
$$


- for $h=n_{a}+1, \ldots, n_{a}+n_{b}$, it holds that

$$
\begin{aligned}
\sigma_{h}^{2}= & \mathrm{E}_{h-1}\left[\left\{\frac{n_{c} n_{d}}{r_{p}} \operatorname{tr}\left(\boldsymbol{T}_{a}\left(n_{a}\right)\left(\boldsymbol{y}_{h-n_{a}} \boldsymbol{y}_{h-n_{a}}^{\prime}-\boldsymbol{\Sigma}_{b}\right) \boldsymbol{\Sigma}_{c} \boldsymbol{\Sigma}_{d}\right)\right\}^{2}\right] \\
= & \frac{n_{c}^{2} n_{d}^{2}}{r_{p}^{2}} \mathrm{E}_{h-1}\left[\left\{\operatorname{tr}\left(\boldsymbol{y}_{h-n_{a}}^{\prime} \boldsymbol{\Sigma}_{b}^{-\frac{1}{2}} \boldsymbol{\Sigma}_{b}^{\frac{1}{2}} \boldsymbol{\Sigma}_{c} \boldsymbol{\Sigma}_{d} \boldsymbol{T}_{a}\left(n_{a}\right) \boldsymbol{\Sigma}_{b}^{\frac{1}{2}} \boldsymbol{\Sigma}_{b}^{-\frac{1}{2}} \boldsymbol{y}_{h-n_{a}}\right)\right.\right. \\
& \left.\left.-\operatorname{tr}\left(\boldsymbol{\Sigma}_{b}^{\frac{1}{2}} \boldsymbol{\Sigma}_{c} \boldsymbol{\Sigma}_{d} \boldsymbol{T}_{a}\left(n_{a}\right) \boldsymbol{\Sigma}_{b}^{\frac{1}{2}}\right)\right\}^{2}\right] \\
= & \frac{n_{c}^{2} n_{d}^{2}}{r_{p}^{2}}\left\{\operatorname{tr}\left(\left(\boldsymbol{\Sigma}_{b}^{\frac{1}{2}} \boldsymbol{\Sigma}_{c} \boldsymbol{\Sigma}_{d} \boldsymbol{T}_{a}\left(n_{a}\right) \boldsymbol{\Sigma}_{b}^{\frac{1}{2}}\right)\left(\boldsymbol{\Sigma}_{b}^{\frac{1}{2}} \boldsymbol{\Sigma}_{c} \boldsymbol{\Sigma}_{d} \boldsymbol{T}_{a}\left(n_{a}\right) \boldsymbol{\Sigma}_{b}^{\frac{1}{2}}\right)\right)\right. \\
& \left.+\operatorname{tr}\left(\left(\boldsymbol{\Sigma}_{b}^{\frac{1}{2}} \boldsymbol{\Sigma}_{c} \boldsymbol{\Sigma}_{d} \boldsymbol{T}_{a}\left(n_{a}\right) \boldsymbol{\Sigma}_{b}^{\frac{1}{2}}\right)\left(\boldsymbol{\Sigma}_{b}^{\frac{1}{2}} \boldsymbol{T}_{a}\left(n_{a}\right) \boldsymbol{\Sigma}_{d} \boldsymbol{\Sigma}_{c} \boldsymbol{\Sigma}_{b}^{\frac{1}{2}}\right)\right)\right\} \\
= & \frac{n_{c}^{2} n_{d}^{2}}{r_{p}^{2}}\left\{\operatorname{tr}\left(\boldsymbol{T}_{a}\left(n_{a}\right) \boldsymbol{\Sigma}_{b} \boldsymbol{\Sigma}_{c} \boldsymbol{\Sigma}_{d} \boldsymbol{T}_{a}\left(n_{a}\right) \boldsymbol{\Sigma}_{b} \boldsymbol{\Sigma}_{c} \boldsymbol{\Sigma}_{d}\right)\right. \\
& \left.+\operatorname{tr}\left(\boldsymbol{T}_{a}\left(n_{a}\right) \boldsymbol{\Sigma}_{b} \boldsymbol{T}_{a}\left(n_{a}\right) \boldsymbol{\Sigma}_{d} \boldsymbol{\Sigma}_{c} \boldsymbol{\Sigma}_{b} \boldsymbol{\Sigma}_{c} \boldsymbol{\Sigma}_{d}\right)\right\}
\end{aligned}
$$

- for $h=n_{a}+n_{b}+1, \ldots, n_{a}+n_{b}+n_{c}$, it holds that

$$
\begin{aligned}
\sigma_{h}^{2}= & \mathrm{E}_{h-1}\left[\left\{\frac{n_{d}}{r_{p}} \operatorname{tr}\left(\boldsymbol{T}_{a}\left(n_{a}\right) \boldsymbol{T}_{b}\left(n_{b}\right)\left(\boldsymbol{z}_{h-n_{a}-n_{b}} \boldsymbol{z}_{h-n_{a}-n_{b}}^{\prime}-\boldsymbol{\Sigma}_{c}\right) \boldsymbol{\Sigma}_{d}\right)\right\}^{2}\right] \\
= & \frac{n_{d}^{2}}{r_{p}^{2}} \mathrm{E}_{h-1}\left[\left\{\operatorname{tr}\left(\boldsymbol{z}_{h-n_{a}-n_{b}}^{\prime} \boldsymbol{\Sigma}_{c}^{-\frac{1}{2}} \boldsymbol{\Sigma}_{c}^{\frac{1}{2}} \boldsymbol{\Sigma}_{d} \boldsymbol{T}_{a}\left(n_{a}\right) \boldsymbol{T}_{b}\left(n_{b}\right) \boldsymbol{\Sigma}_{c}^{\frac{1}{2}} \boldsymbol{\Sigma}_{c}^{-\frac{1}{2}} \boldsymbol{z}_{h-n_{a}-n_{b}}\right)\right.\right. \\
& \left.\left.-\operatorname{tr}\left(\boldsymbol{\Sigma}_{c}^{\frac{1}{2}} \boldsymbol{\Sigma}_{d} \boldsymbol{T}_{a}\left(n_{a}\right) \boldsymbol{T}_{b}\left(n_{b}\right) \boldsymbol{\Sigma}_{c}^{\frac{1}{2}}\right)\right\}^{2}\right] \\
= & \frac{n_{d}^{2}}{r_{p}^{2}}\left\{\operatorname{tr}\left(\boldsymbol{\Sigma}_{c}^{\frac{1}{2}} \boldsymbol{\Sigma}_{d} \boldsymbol{T}_{a}\left(n_{a}\right) \boldsymbol{T}_{b}\left(n_{b}\right) \boldsymbol{\Sigma}_{c}^{\frac{1}{2}} \boldsymbol{\Sigma}_{c}^{\frac{1}{2}} \boldsymbol{\Sigma}_{d} \boldsymbol{T}_{a}\left(n_{a}\right) \boldsymbol{T}_{b}\left(n_{b}\right) \boldsymbol{\Sigma}_{c}^{\frac{1}{2}}\right)\right. \\
& \left.+\operatorname{tr}\left(\boldsymbol{\Sigma}_{c}^{\frac{1}{2}} \boldsymbol{\Sigma}_{d} \boldsymbol{T}_{a}\left(n_{a}\right) \boldsymbol{T}_{b}\left(n_{b}\right) \boldsymbol{\Sigma}_{c}^{\frac{1}{2}} \boldsymbol{\Sigma}_{c}^{\frac{1}{2}} \boldsymbol{T}_{b}\left(n_{b}\right) \boldsymbol{T}_{a}\left(n_{a}\right) \boldsymbol{\Sigma}_{d} \boldsymbol{\Sigma}_{c}^{\frac{1}{2}}\right)\right\} \\
= & \frac{n_{d}^{2}}{r_{p}^{2}}\left\{\operatorname{tr}\left(\boldsymbol{T}_{a}\left(n_{a}\right) \boldsymbol{T}_{b}\left(n_{b}\right) \boldsymbol{\Sigma}_{c} \boldsymbol{\Sigma}_{d} \boldsymbol{T}_{a}\left(n_{a}\right) \boldsymbol{T}_{b}\left(n_{b}\right) \boldsymbol{\Sigma}_{c} \boldsymbol{\Sigma}_{d}\right)\right. \\
& \left.+\operatorname{tr}\left(\boldsymbol{T}_{a}\left(n_{a}\right) \boldsymbol{T}_{b}\left(n_{b}\right) \boldsymbol{\Sigma}_{c} \boldsymbol{T}_{b}\left(n_{b}\right) \boldsymbol{T}_{a}\left(n_{a}\right) \boldsymbol{\Sigma}_{d} \boldsymbol{\Sigma}_{c} \boldsymbol{\Sigma}_{d}\right)\right\}
\end{aligned}
$$

- for $h=n_{a}+n_{b}+n_{c}+1, \ldots, n_{a}+n_{b}+n_{c}+n_{d}$, it holds that

$$
\begin{aligned}
\sigma_{h}^{2}= & \mathrm{E}_{h-1}\left[\left\{\frac{1}{r_{p}} \operatorname{tr}\left(\boldsymbol{T}_{a}\left(n_{a}\right) \boldsymbol{T}_{b}\left(n_{b}\right) \boldsymbol{T}_{c}\left(n_{c}\right)\left(\boldsymbol{w}_{h-n_{a}-n_{b}-n_{c}} \boldsymbol{w}_{h-n_{a}-n_{b}-n_{c}}^{\prime}-\boldsymbol{\Sigma}_{d}\right)\right)\right\}^{2}\right] \\
= & \frac{1}{r_{p}^{2}} \mathrm{E}_{h-1}\left[\left\{\operatorname{tr}\left(\boldsymbol{w}_{h-n_{a}-n_{b}-n_{c}}^{\prime} \boldsymbol{\Sigma}_{d}^{-\frac{1}{2}} \boldsymbol{\Sigma}_{d}^{\frac{1}{2}} \boldsymbol{T}_{a}\left(n_{a}\right) \boldsymbol{T}_{b}\left(n_{b}\right) \boldsymbol{T}_{c}\left(n_{c}\right) \boldsymbol{\Sigma}_{d}^{\frac{1}{2}} \boldsymbol{\Sigma}_{d}^{-\frac{1}{2}} \boldsymbol{w}_{h-n_{a}-n_{b}-n_{c}}\right)\right.\right. \\
& \left.\left.-\operatorname{tr}\left(\boldsymbol{\Sigma}_{d}^{\frac{1}{2}} \boldsymbol{T}_{a}\left(n_{a}\right) \boldsymbol{T}_{b}\left(n_{b}\right) \boldsymbol{T}_{c}\left(n_{c}\right) \boldsymbol{\Sigma}_{d}^{\frac{1}{2}}\right)\right\}^{2}\right] \\
= & \frac{1}{r_{p}^{2}}\left\{\operatorname{tr}\left(\boldsymbol{\Sigma}_{d}^{\frac{1}{2}} \boldsymbol{T}_{a}\left(n_{a}\right) \boldsymbol{T}_{b}\left(n_{b}\right) \boldsymbol{T}_{c}\left(n_{c}\right) \boldsymbol{\Sigma}_{d}^{\frac{1}{2}} \boldsymbol{\Sigma}_{d}^{\frac{1}{2}} \boldsymbol{T}_{a}\left(n_{a}\right) \boldsymbol{T}_{b}\left(n_{b}\right) \boldsymbol{T}_{c}\left(n_{c}\right) \boldsymbol{\Sigma}_{d}^{\frac{1}{2}}\right)\right. \\
& \left.+\operatorname{tr}\left(\boldsymbol{\Sigma}_{d}^{\frac{1}{2}} \boldsymbol{T}_{a}\left(n_{a}\right) \boldsymbol{T}_{b}\left(n_{b}\right) \boldsymbol{T}_{c}\left(n_{c}\right) \boldsymbol{\Sigma}_{d}^{\frac{1}{2}} \boldsymbol{\Sigma}_{d}^{\frac{1}{2}} \boldsymbol{T}_{c}\left(n_{c}\right) \boldsymbol{T}_{b}\left(n_{b}\right) \boldsymbol{T}_{a}\left(n_{a}\right) \boldsymbol{\Sigma}_{d}^{\frac{1}{2}}\right)\right\} \\
= & \frac{1}{r_{p}^{2}}\left\{\operatorname{tr}\left(\boldsymbol{T}_{a}\left(n_{a}\right) \boldsymbol{T}_{b}\left(n_{b}\right) \boldsymbol{T}_{c}\left(n_{c}\right) \boldsymbol{\Sigma}_{d} \boldsymbol{T}_{a}\left(n_{a}\right) \boldsymbol{T}_{b}\left(n_{b}\right) \boldsymbol{T}_{c}\left(n_{c}\right) \boldsymbol{\Sigma}_{d}\right)\right. \\
& \left.+\operatorname{tr}\left(\boldsymbol{T}_{a}\left(n_{a}\right) \boldsymbol{T}_{b}\left(n_{b}\right) \boldsymbol{T}_{c}\left(n_{c}\right) \boldsymbol{\Sigma}_{d} \boldsymbol{T}_{c}\left(n_{c}\right) \boldsymbol{T}_{b}\left(n_{b}\right) \boldsymbol{T}_{a}\left(n_{a}\right) \boldsymbol{\Sigma}_{d}\right)\right\} .
\end{aligned}
$$

In general, as

$$
\begin{aligned}
\mathrm{V}[X+Y] & =\mathrm{V}[X]+\mathrm{V}[Y]+2 \operatorname{Cov}[X, Y] \leq \mathrm{V}[X]+\mathrm{V}[Y]+2(\mathrm{~V}[X] \mathrm{V}[Y])^{\frac{1}{2}} \\
& \leq \mathrm{V}[X]+\mathrm{V}[Y]+2 \max (\mathrm{V}[X], \mathrm{V}[Y]) \leq 4 \max (\mathrm{V}[X], \mathrm{V}[Y])
\end{aligned}
$$


for random variables $X$ and $Y$ which have finite second moments, in order to prove

$$
\mathrm{V}\left[\sum_{h=1}^{n_{a}+n_{b}+n_{c}+n_{d}} \sigma_{h}^{2}\right] \rightarrow 0,
$$

it suffices to show

$$
\begin{gathered}
\mathrm{V}\left[\sum_{h=1}^{n_{a}} \sigma_{h}^{2}\right] \rightarrow 0, \\
\mathrm{~V}\left[\sum_{h=n_{a}+1}^{n_{a}+n_{b}} \sigma_{h}^{2}\right] \rightarrow 0, \\
\mathrm{~V}\left[\sum_{h=n_{a}+n_{b}+1}^{n_{a}+n_{b}+n_{c}} \sigma_{h}^{2}\right] \rightarrow 0,
\end{gathered}
$$

and

$$
\mathrm{V}\left[\sum_{h=n_{a}+n_{b}+n_{c}+1}^{n_{a}+n_{b}+n_{c}+n_{d}} \sigma_{h}^{2}\right] \rightarrow 0 .
$$

- Proof of (5.1). As $r_{p}=p^{2} \sqrt{n_{a} n_{b} n_{c} n_{d}}$, it follows from

$$
\sum_{h=1}^{n_{a}} \sigma_{h}^{2}=\frac{n_{b} n_{c} n_{d}}{p^{4}}\left\{\operatorname{tr}\left(\boldsymbol{\Sigma}_{a} \boldsymbol{\Sigma}_{b} \boldsymbol{\Sigma}_{c} \boldsymbol{\Sigma}_{d} \boldsymbol{\Sigma}_{a} \boldsymbol{\Sigma}_{b} \boldsymbol{\Sigma}_{c} \boldsymbol{\Sigma}_{d}\right)+\operatorname{tr}\left(\boldsymbol{\Sigma}_{a} \boldsymbol{\Sigma}_{b} \boldsymbol{\Sigma}_{c} \boldsymbol{\Sigma}_{d} \boldsymbol{\Sigma}_{a} \boldsymbol{\Sigma}_{d} \boldsymbol{\Sigma}_{c} \boldsymbol{\Sigma}_{b}\right)\right\}
$$

that

$$
\mathrm{E}\left[\left(\sum_{h=1}^{n_{a}} \sigma_{h}^{2}\right)^{2}\right]=O\left(\frac{n_{b}^{2} n_{c}^{2} n_{d}^{2}}{p^{6}}\right) \rightarrow 0
$$

- Proof of (5.2). It follows from

$$
\begin{aligned}
\sum_{h=n_{a}+1}^{n_{a}+n_{b}} \sigma_{h}^{2}= & \frac{n_{c} n_{d}}{p^{4} n_{a}}\left\{\operatorname{tr}\left(\boldsymbol{T}_{a}\left(n_{a}\right) \boldsymbol{\Sigma}_{b} \boldsymbol{\Sigma}_{c} \boldsymbol{\Sigma}_{d} \boldsymbol{T}_{a}\left(n_{a}\right) \boldsymbol{\Sigma}_{b} \boldsymbol{\Sigma}_{c} \boldsymbol{\Sigma}_{d}\right)\right. \\
& \left.+\operatorname{tr}\left(\boldsymbol{T}_{a}\left(n_{a}\right) \boldsymbol{\Sigma}_{b} \boldsymbol{T}_{a}\left(n_{a}\right) \boldsymbol{\Sigma}_{d} \boldsymbol{\Sigma}_{c} \boldsymbol{\Sigma}_{b} \boldsymbol{\Sigma}_{c} \boldsymbol{\Sigma}_{d}\right)\right\}
\end{aligned}
$$

that

$$
\mathrm{E}\left[\left(\sum_{h=n_{a}+1}^{n_{a}+n_{b}} \sigma_{h}^{2}\right)^{2}\right]=\frac{n_{c}^{2} n_{d}^{2}}{p^{8} n_{a}^{2}} O\left(n_{a}^{2} p^{4}\right)=O\left(\frac{n_{c}^{2} n_{d}^{2}}{p^{4}}\right) \rightarrow 0 .
$$

- Proof of (5.3). By using Proposition 4.8, it follows from

$$
\begin{aligned}
\sum_{h=n_{a}+n_{b}+1}^{n_{a}+n_{b}+n_{c}} \sigma_{h}^{2}= & \frac{n_{d}}{p^{4} n_{a} n_{b}}\left\{\operatorname{tr}\left(\boldsymbol{T}_{a}\left(n_{a}\right) \boldsymbol{T}_{b}\left(n_{b}\right) \boldsymbol{\Sigma}_{c} \boldsymbol{\Sigma}_{d} \boldsymbol{T}_{a}\left(n_{a}\right) \boldsymbol{T}_{b}\left(n_{b}\right) \boldsymbol{\Sigma}_{c} \boldsymbol{\Sigma}_{d}\right)\right. \\
& \left.+\operatorname{tr}\left(\boldsymbol{T}_{a}\left(n_{a}\right) \boldsymbol{T}_{b}\left(n_{b}\right) \boldsymbol{\Sigma}_{c} \boldsymbol{T}_{b}\left(n_{b}\right) \boldsymbol{T}_{a}\left(n_{a}\right) \boldsymbol{\Sigma}_{d} \boldsymbol{\Sigma}_{c} \boldsymbol{\Sigma}_{d}\right)\right\}
\end{aligned}
$$

that

$$
\mathrm{E}\left[\left(\sum_{h=n_{a}+n_{b}+1}^{n_{a}+n_{b}+n_{c}} \sigma_{h}^{2}\right)^{2}\right]=\frac{n_{d}^{2}}{p^{8} n_{a}^{2} n_{b}^{2}} O\left(n_{a}^{2} n_{b}^{2} p^{6}\right)=O\left(\frac{n_{d}^{2}}{p^{2}}\right) \rightarrow 0 .
$$

- Proof of (5.4). It follows from

$$
\begin{aligned}
& \sum_{h=n_{a}+n_{b}+n_{c}+1}^{n_{a}+n_{b}+n_{c}+n_{d}} \sigma_{h}^{2} \\
& =\frac{1}{p^{4} n_{a} n_{b} n_{c}}\left\{\operatorname{tr}\left(\boldsymbol{T}_{a}\left(n_{a}\right) \boldsymbol{T}_{b}\left(n_{b}\right) \boldsymbol{T}_{c}\left(n_{c}\right) \boldsymbol{\Sigma}_{d} \boldsymbol{T}_{a}\left(n_{a}\right) \boldsymbol{T}_{b}\left(n_{b}\right) \boldsymbol{T}_{c}\left(n_{c}\right) \boldsymbol{\Sigma}_{d}\right)\right. \\
& \left.\quad+\operatorname{tr}\left(\boldsymbol{T}_{a}\left(n_{a}\right) \boldsymbol{T}_{b}\left(n_{b}\right) \boldsymbol{T}_{c}\left(n_{c}\right) \boldsymbol{\Sigma}_{d} \boldsymbol{T}_{c}\left(n_{c}\right) \boldsymbol{T}_{b}\left(n_{b}\right) \boldsymbol{T}_{a}\left(n_{a}\right) \boldsymbol{\Sigma}_{d}\right)\right\}
\end{aligned}
$$


that

$$
\begin{aligned}
& \mathrm{E}\left[\left(\sum_{h=n_{a}+n_{b}+n_{c}+1}^{n_{a}+n_{b}+n_{c}+n_{d}} \sigma_{h}^{2}\right)^{2}\right] \\
& =\frac{1}{p^{8} n_{a}^{2} n_{b}^{2} n_{c}^{2}} \mathrm{E}\left[\left\{\operatorname{tr}\left(\boldsymbol{T}_{a}\left(n_{a}\right) \boldsymbol{T}_{b}\left(n_{b}\right) \boldsymbol{T}_{c}\left(n_{c}\right) \boldsymbol{\Sigma}_{d} \boldsymbol{T}_{a}\left(n_{a}\right) \boldsymbol{T}_{b}\left(n_{b}\right) \boldsymbol{T}_{c}\left(n_{c}\right) \boldsymbol{\Sigma}_{d}\right)\right.\right. \\
& \left.\left.+\operatorname{tr}\left(\boldsymbol{T}_{a}\left(n_{a}\right) \boldsymbol{T}_{b}\left(n_{b}\right) \boldsymbol{T}_{c}\left(n_{c}\right) \boldsymbol{\Sigma}_{d} \boldsymbol{T}_{c}\left(n_{c}\right) \boldsymbol{T}_{b}\left(n_{b}\right) \boldsymbol{T}_{a}\left(n_{a}\right) \boldsymbol{\Sigma}_{d}\right)\right\}^{2}\right] \\
& =\frac{1}{p^{8} n_{a}^{2} n_{b}^{2} n_{c}^{2}} \times \\
& \mathrm{E}\left[\operatorname{tr}\left(\boldsymbol{T}_{a}\left(n_{a}\right) \boldsymbol{T}_{b}\left(n_{b}\right) \boldsymbol{T}_{c}\left(n_{c}\right) \boldsymbol{\Sigma}_{d} \boldsymbol{T}_{a}\left(n_{a}\right) \boldsymbol{T}_{b}\left(n_{b}\right) \boldsymbol{T}_{c}\left(n_{c}\right) \boldsymbol{\Sigma}_{d}\right)\right. \\
& \times \operatorname{tr}\left(\boldsymbol{T}_{a}\left(n_{a}\right) \boldsymbol{T}_{b}\left(n_{b}\right) \boldsymbol{T}_{c}\left(n_{c}\right) \boldsymbol{\Sigma}_{d} \boldsymbol{T}_{a}\left(n_{a}\right) \boldsymbol{T}_{b}\left(n_{b}\right) \boldsymbol{T}_{c}\left(n_{c}\right) \boldsymbol{\Sigma}_{d}\right) \\
& +\operatorname{tr}\left(\boldsymbol{T}_{a}\left(n_{a}\right) \boldsymbol{T}_{b}\left(n_{b}\right) \boldsymbol{T}_{c}\left(n_{c}\right) \boldsymbol{\Sigma}_{d} \boldsymbol{T}_{a}\left(n_{a}\right) \boldsymbol{T}_{b}\left(n_{b}\right) \boldsymbol{T}_{c}\left(n_{c}\right) \boldsymbol{\Sigma}_{d}\right) \\
& \times \operatorname{tr}\left(\boldsymbol{T}_{a}\left(n_{a}\right) \boldsymbol{T}_{b}\left(n_{b}\right) \boldsymbol{T}_{c}\left(n_{c}\right) \boldsymbol{\Sigma}_{d} \boldsymbol{T}_{c}\left(n_{c}\right) \boldsymbol{T}_{b}\left(n_{b}\right) \boldsymbol{T}_{a}\left(n_{a}\right) \boldsymbol{\Sigma}_{d}\right) \\
& +\operatorname{tr}\left(\boldsymbol{T}_{a}\left(n_{a}\right) \boldsymbol{T}_{b}\left(n_{b}\right) \boldsymbol{T}_{c}\left(n_{c}\right) \boldsymbol{\Sigma}_{d} \boldsymbol{T}_{c}\left(n_{c}\right) \boldsymbol{T}_{b}\left(n_{b}\right) \boldsymbol{T}_{a}\left(n_{a}\right) \boldsymbol{\Sigma}_{d}\right) \\
& \times \operatorname{tr}\left(\boldsymbol{T}_{a}\left(n_{a}\right) \boldsymbol{T}_{b}\left(n_{b}\right) \boldsymbol{T}_{c}\left(n_{c}\right) \boldsymbol{\Sigma}_{d} \boldsymbol{T}_{a}\left(n_{a}\right) \boldsymbol{T}_{b}\left(n_{b}\right) \boldsymbol{T}_{c}\left(n_{c}\right) \boldsymbol{\Sigma}_{d}\right) \\
& +\operatorname{tr}\left(\boldsymbol{T}_{a}\left(n_{a}\right) \boldsymbol{T}_{b}\left(n_{b}\right) \boldsymbol{T}_{c}\left(n_{c}\right) \boldsymbol{\Sigma}_{d} \boldsymbol{T}_{c}\left(n_{c}\right) \boldsymbol{T}_{b}\left(n_{b}\right) \boldsymbol{T}_{a}\left(n_{a}\right) \boldsymbol{\Sigma}_{d}\right) \\
& \left.\times \operatorname{tr}\left(\boldsymbol{T}_{a}\left(n_{a}\right) \boldsymbol{T}_{b}\left(n_{b}\right) \boldsymbol{T}_{c}\left(n_{c}\right) \boldsymbol{\Sigma}_{d} \boldsymbol{T}_{c}\left(n_{c}\right) \boldsymbol{T}_{b}\left(n_{b}\right) \boldsymbol{T}_{a}\left(n_{a}\right) \boldsymbol{\Sigma}_{d}\right)\right] \\
& =\frac{1}{p^{8} n_{b}^{2} n_{c}^{2}} \mathrm{E}\left[\operatorname{tr}\left(\boldsymbol{\Sigma}_{a} \boldsymbol{T}_{b}\left(n_{b}\right) \boldsymbol{T}_{c}\left(n_{c}\right) \boldsymbol{\Sigma}_{d}\right) \operatorname{tr}\left(\boldsymbol{\Sigma}_{a} \boldsymbol{T}_{b}\left(n_{b}\right) \boldsymbol{T}_{c}\left(n_{c}\right) \boldsymbol{\Sigma}_{d}\right)\right. \\
& \times \operatorname{tr}\left(\boldsymbol{\Sigma}_{a} \boldsymbol{T}_{b}\left(n_{b}\right) \boldsymbol{T}_{c}\left(n_{c}\right) \boldsymbol{\Sigma}_{d}\right) \operatorname{tr}\left(\boldsymbol{\Sigma}_{a} \boldsymbol{T}_{b}\left(n_{b}\right) \boldsymbol{T}_{c}\left(n_{c}\right) \boldsymbol{\Sigma}_{d}\right) \\
& +\operatorname{tr}\left(\boldsymbol{\Sigma}_{a} \boldsymbol{T}_{b}\left(n_{b}\right) \boldsymbol{T}_{c}\left(n_{c}\right) \boldsymbol{\Sigma}_{d}\right) \operatorname{tr}\left(\boldsymbol{\Sigma}_{a} \boldsymbol{T}_{b}\left(n_{b}\right) \boldsymbol{T}_{c}\left(n_{c}\right) \boldsymbol{\Sigma}_{d}\right) \\
& \times \operatorname{tr}\left(\boldsymbol{\Sigma}_{a} \boldsymbol{T}_{b}\left(n_{b}\right) \boldsymbol{T}_{c}\left(n_{c}\right) \boldsymbol{\Sigma}_{d} \boldsymbol{T}_{c}\left(n_{c}\right) \boldsymbol{T}_{b}\left(n_{b}\right)\right) \operatorname{tr}\left(\boldsymbol{\Sigma}_{a} \boldsymbol{\Sigma}_{d}\right) \\
& +\operatorname{tr}\left(\boldsymbol{\Sigma}_{a} \boldsymbol{T}_{b}\left(n_{b}\right) \boldsymbol{T}_{c}\left(n_{c}\right) \boldsymbol{\Sigma}_{d} \boldsymbol{T}_{c}\left(n_{c}\right) \boldsymbol{T}_{b}\left(n_{b}\right)\right) \operatorname{tr}\left(\boldsymbol{\Sigma}_{a} \boldsymbol{\Sigma}_{d}\right) \\
& \times \operatorname{tr}\left(\boldsymbol{\Sigma}_{a} \boldsymbol{T}_{b}\left(n_{b}\right) \boldsymbol{T}_{c}\left(n_{c}\right) \boldsymbol{\Sigma}_{d}\right) \operatorname{tr}\left(\boldsymbol{\Sigma}_{a} \boldsymbol{T}_{b}\left(n_{b}\right) \boldsymbol{T}_{c}\left(n_{c}\right) \boldsymbol{\Sigma}_{d}\right) \\
& +\operatorname{tr}\left(\boldsymbol{\Sigma}_{a} \boldsymbol{T}_{b}\left(n_{b}\right) \boldsymbol{T}_{c}\left(n_{c}\right) \boldsymbol{\Sigma}_{d} \boldsymbol{T}_{c}\left(n_{c}\right) \boldsymbol{T}_{b}\left(n_{b}\right)\right) \operatorname{tr}\left(\boldsymbol{\Sigma}_{a} \boldsymbol{\Sigma}_{d}\right) \\
& \left.\times \operatorname{tr}\left(\boldsymbol{\Sigma}_{a} \boldsymbol{T}_{b}\left(n_{b}\right) \boldsymbol{T}_{c}\left(n_{c}\right) \boldsymbol{\Sigma}_{d} \boldsymbol{T}_{c}\left(n_{c}\right) \boldsymbol{T}_{b}\left(n_{b}\right)\right) \operatorname{tr}\left(\boldsymbol{\Sigma}_{a} \boldsymbol{\Sigma}_{d}\right)\right]+o(1) \\
& =\frac{1}{p^{8} n_{c}^{2}} \mathrm{E}\left[\operatorname{tr}\left(\boldsymbol{\Sigma}_{b} \boldsymbol{T}_{c}\left(n_{c}\right) \boldsymbol{\Sigma}_{d} \boldsymbol{T}_{c}\left(n_{c}\right)\right) \operatorname{tr}\left(\boldsymbol{\Sigma}_{b} \boldsymbol{\Sigma}_{a}\right) \operatorname{tr}\left(\boldsymbol{\Sigma}_{a} \boldsymbol{\Sigma}_{d}\right)\right. \\
& \left.\times \operatorname{tr}\left(\boldsymbol{\Sigma}_{b} \boldsymbol{T}_{c}\left(n_{c}\right) \boldsymbol{\Sigma}_{d} \boldsymbol{T}_{c}\left(n_{c}\right)\right) \operatorname{tr}\left(\boldsymbol{\Sigma}_{b} \boldsymbol{\Sigma}_{a}\right) \operatorname{tr}\left(\boldsymbol{\Sigma}_{a} \boldsymbol{\Sigma}_{d}\right)\right]+o(1) \\
& =\frac{1}{p^{8}} \operatorname{tr}\left(\boldsymbol{\Sigma}_{c} \boldsymbol{\Sigma}_{d}\right) \operatorname{tr}\left(\boldsymbol{\Sigma}_{c} \boldsymbol{\Sigma}_{b}\right) \operatorname{tr}\left(\boldsymbol{\Sigma}_{b} \boldsymbol{\Sigma}_{a}\right) \operatorname{tr}\left(\boldsymbol{\Sigma}_{a} \boldsymbol{\Sigma}_{d}\right) \\
& \times \operatorname{tr}\left(\boldsymbol{\Sigma}_{c} \boldsymbol{\Sigma}_{d}\right) \operatorname{tr}\left(\boldsymbol{\Sigma}_{c} \boldsymbol{\Sigma}_{b}\right) \operatorname{tr}\left(\boldsymbol{\Sigma}_{b} \boldsymbol{\Sigma}_{a}\right) \operatorname{tr}\left(\boldsymbol{\Sigma}_{a} \boldsymbol{\Sigma}_{d}\right)+o(1) \\
& \rightarrow \sigma_{a b}^{2} \sigma_{a d}^{2} \sigma_{b c}^{2} \sigma_{c d}^{2} \text {. }
\end{aligned}
$$

and that

$$
\begin{aligned}
\mathrm{E}[ & \left.\sum_{h=n_{a}+n_{b}+n_{c}+1}^{n_{a}+n_{b}+n_{c}+n_{d}} \sigma_{h}^{2}\right] \\
= & \frac{1}{p^{4} n_{a} n_{b} n_{c}} \mathrm{E}\left[\operatorname{tr}\left(\boldsymbol{T}_{a}\left(n_{a}\right) \boldsymbol{T}_{b}\left(n_{b}\right) \boldsymbol{T}_{c}\left(n_{c}\right) \boldsymbol{\Sigma}_{d} \boldsymbol{T}_{a}\left(n_{a}\right) \boldsymbol{T}_{b}\left(n_{b}\right) \boldsymbol{T}_{c}\left(n_{c}\right) \boldsymbol{\Sigma}_{d}\right)\right. \\
& \left.+\operatorname{tr}\left(\boldsymbol{T}_{a}\left(n_{a}\right) \boldsymbol{T}_{b}\left(n_{b}\right) \boldsymbol{T}_{c}\left(n_{c}\right) \boldsymbol{\Sigma}_{d} \boldsymbol{T}_{c}\left(n_{c}\right) \boldsymbol{T}_{b}\left(n_{b}\right) \boldsymbol{T}_{a}\left(n_{a}\right) \boldsymbol{\Sigma}_{d}\right)\right] \\
= & \frac{1}{p^{4} n_{b} n_{c}} \mathrm{E}\left[\operatorname{tr}\left(\boldsymbol{\Sigma}_{a} \boldsymbol{T}_{b}\left(n_{b}\right) \boldsymbol{T}_{c}\left(n_{c}\right) \boldsymbol{\Sigma}_{d}\right) \operatorname{tr}\left(\boldsymbol{\Sigma}_{a} \boldsymbol{T}_{b}\left(n_{b}\right) \boldsymbol{T}_{c}\left(n_{c}\right) \boldsymbol{\Sigma}_{d}\right)\right. \\
& \left.+\operatorname{tr}\left(\boldsymbol{\Sigma}_{a} \boldsymbol{T}_{b}\left(n_{b}\right) \boldsymbol{T}_{c}\left(n_{c}\right) \boldsymbol{\Sigma}_{d} \boldsymbol{T}_{c}\left(n_{c}\right) \boldsymbol{T}_{b}\left(n_{b}\right)\right) \operatorname{tr}\left(\boldsymbol{\Sigma}_{a} \boldsymbol{\Sigma}_{d}\right)\right]+o(1) \\
= & \frac{1}{p^{4} n_{c}} \mathrm{E}\left[\operatorname{tr}\left(\boldsymbol{\Sigma}_{b} \boldsymbol{T}_{c}\left(n_{c}\right) \boldsymbol{\Sigma}_{d} \boldsymbol{T}_{c}\left(n_{c}\right)\right) \operatorname{tr}\left(\boldsymbol{\Sigma}_{b} \boldsymbol{\Sigma}_{a}\right) \operatorname{tr}\left(\boldsymbol{\Sigma}_{a} \boldsymbol{\Sigma}_{d}\right)\right]+o(1) \\
= & \frac{1}{p^{4}} \mathrm{E}\left[\operatorname{tr}\left(\boldsymbol{\Sigma}_{b} \boldsymbol{\Sigma}_{c}\right) \operatorname{tr}\left(\boldsymbol{\Sigma}_{d} \boldsymbol{\Sigma}_{c}\right) \operatorname{tr}\left(\boldsymbol{\Sigma}_{b} \boldsymbol{\Sigma}_{a}\right) \operatorname{tr}\left(\boldsymbol{\Sigma}_{a} \boldsymbol{\Sigma}_{d}\right)\right]+o(1) \\
\rightarrow & \sigma_{a b} \sigma_{a d} \sigma_{b c} \sigma_{c d} .
\end{aligned}
$$


Hence,

$$
\begin{aligned}
\mathrm{V} & {\left[\sum_{h=n_{a}+n_{b}+n_{c}+1}^{n_{a}+n_{b}+n_{c}+n_{d}} \sigma_{h}^{2}\right] } \\
& =\mathrm{E}\left[\left(\sum_{h=n_{a}+n_{b}+n_{c}+1}^{n_{a}+n_{b}+n_{c}+n_{d}} \sigma_{h}^{2}\right)^{2}\right]-\left(\mathrm{E}\left[\sum_{h=n_{a}+n_{b}+n_{c}+1}^{n_{a}+n_{b}+n_{c}+n_{d}} \sigma_{h}^{2}\right]\right)^{2} \\
& \rightarrow 0
\end{aligned}
$$

This completes the proof of Lemma 2.3.

\subsection{Proof of Lemma 2.4}

To prove

$$
\sum_{h=1}^{n_{a}+n_{b}+n_{c}+n_{d}} \mathrm{E}\left[D_{h}^{4}\right] \rightarrow 0
$$

it suffices to show

$$
\begin{gathered}
\sum_{h=1}^{n_{a}} \mathrm{E}\left[D_{h}^{4}\right] \rightarrow 0, \\
\sum_{h=n_{a}+1}^{n_{a}+n_{b}} \mathrm{E}\left[D_{h}^{4}\right] \rightarrow 0, \\
\sum_{h=n_{a}+n_{b}+1}^{n_{a}+n_{b}+n_{c}} \mathrm{E}\left[D_{h}^{4}\right] \rightarrow 0,
\end{gathered}
$$

and

$$
\sum_{h=n_{a}+n_{b}+n_{c}+1}^{n_{a}+n_{b}+n_{c}+n_{d}} \mathrm{E}\left[D_{h}^{4}\right] \rightarrow 0 .
$$

- Proof of (5.5). For $h=1, \ldots, n_{a}$, it follows from Lemma 4.3 that

$$
\begin{aligned}
\mathrm{E}\left[D_{h}^{4}\right] & =\mathrm{E}\left[\left\{\frac{n_{b} n_{c} n_{d}}{r_{p}} \operatorname{tr}\left(\left(\boldsymbol{x}_{h} \boldsymbol{x}_{h}^{\prime}-\boldsymbol{\Sigma}_{a}\right) \boldsymbol{\Sigma}_{b} \boldsymbol{\Sigma}_{c} \boldsymbol{\Sigma}_{d}\right)\right\}^{4}\right] \\
& =\frac{n_{b}^{4} n_{c}^{4} n_{d}^{4}}{r_{p}^{4}} \mathrm{E}\left[\left\{\boldsymbol{x}_{h}^{\prime} \boldsymbol{\Sigma}_{b} \boldsymbol{\Sigma}_{c} \boldsymbol{\Sigma}_{d} \boldsymbol{x}_{h}-\operatorname{tr}\left(\boldsymbol{\Sigma}_{a} \boldsymbol{\Sigma}_{b} \boldsymbol{\Sigma}_{c} \boldsymbol{\Sigma}_{d}\right)\right\}^{4}\right] \\
& =\frac{n_{b}^{4} n_{c}^{4} n_{d}^{4}}{r_{p}^{4}} \mathrm{E}\left[\left\{\boldsymbol{x}_{h}^{\prime} \boldsymbol{\Sigma}_{a}^{-\frac{1}{2}} \boldsymbol{\Sigma}_{a}^{\frac{1}{2}} \boldsymbol{\Sigma}_{b} \boldsymbol{\Sigma}_{c} \boldsymbol{\Sigma}_{d} \boldsymbol{\Sigma}_{a}^{\frac{1}{2}} \boldsymbol{\Sigma}_{a}^{-\frac{1}{2}} \boldsymbol{x}_{h}-\operatorname{tr}\left(\boldsymbol{\Sigma}_{a}^{\frac{1}{2}} \boldsymbol{\Sigma}_{b} \boldsymbol{\Sigma}_{c} \boldsymbol{\Sigma}_{d} \boldsymbol{\Sigma}_{a}^{\frac{1}{2}}\right)\right\}^{4}\right] \\
& =\frac{n_{b}^{4} n_{c}^{4} n_{d}^{4}}{r_{p}^{4}}\left\{3 \operatorname{tr}\left(\left(\boldsymbol{A}+\boldsymbol{A}^{\prime}\right)^{4}\right)+\frac{3}{4}\left\{\operatorname{tr}\left(\left(\boldsymbol{A}+\boldsymbol{A}^{\prime}\right)^{2}\right)\right\}^{2}\right\}
\end{aligned}
$$

where $\boldsymbol{A}=\boldsymbol{\Sigma}_{a}^{\frac{1}{2}} \boldsymbol{\Sigma}_{b} \boldsymbol{\Sigma}_{c} \boldsymbol{\Sigma}_{d} \boldsymbol{\Sigma}_{a}^{\frac{1}{2}}$. In general, for a nonnegative definite matrix $\boldsymbol{B} \neq \mathbf{0}$ whose Spectral decomposition is given by $\boldsymbol{B}=\boldsymbol{U} \boldsymbol{\Lambda} \boldsymbol{U}^{\prime}$, it holds that

$$
\{\operatorname{tr}(\boldsymbol{B})\}^{2}=(\operatorname{tr}(\boldsymbol{\Lambda}))^{2}=\left(\sum_{i=1}^{p} \lambda_{i}\right)^{2}>\sum_{i=1}^{p} \lambda_{i}^{2}=\operatorname{tr}\left(\boldsymbol{\Lambda}^{2}\right)=\operatorname{tr}\left(\boldsymbol{B}^{2}\right) .
$$


This implies that

$$
\begin{aligned}
\sum_{h=1}^{n_{a}} \mathrm{E}\left[D_{h}^{4}\right] & =\frac{n_{a} n_{b}^{4} n_{c}^{4} n_{d}^{4}}{r_{p}^{4}}\left[3 \operatorname{tr}\left(\left(\boldsymbol{A}+\boldsymbol{A}^{\prime}\right)^{4}\right)+\frac{3}{4}\left\{\operatorname{tr}\left(\left(\boldsymbol{A}+\boldsymbol{A}^{\prime}\right)^{2}\right)\right\}^{2}\right] \\
& <\frac{n_{a} n_{b}^{4} n_{c}^{4} n_{d}^{4}}{r_{p}^{4}}\left[3\left\{\operatorname{tr}\left(\left(\boldsymbol{A}+\boldsymbol{A}^{\prime}\right)^{2}\right)\right\}^{2}+\frac{3}{4}\left\{\operatorname{tr}\left(\left(\boldsymbol{A}+\boldsymbol{A}^{\prime}\right)^{2}\right)\right\}^{2}\right] \\
& =\frac{15}{4} \frac{n_{a} n_{b}^{4} n_{c}^{4} n_{d}^{4}}{r_{p}^{4}}\left\{\operatorname{tr}\left(\left(\boldsymbol{A}+\boldsymbol{A}^{\prime}\right)^{2}\right)\right\}^{2} \\
& =\frac{15}{4} \frac{n_{a} n_{b}^{4} n_{c}^{4} n_{d}^{4}}{r_{p}^{4}}\left\{\operatorname{tr}\left(\left(\boldsymbol{\Sigma}_{a}^{\frac{1}{2}} \boldsymbol{\Sigma}_{b} \boldsymbol{\Sigma}_{c} \boldsymbol{\Sigma}_{d} \boldsymbol{\Sigma}_{a}^{\frac{1}{2}}+\boldsymbol{\Sigma}_{a}^{\frac{1}{2}} \boldsymbol{\Sigma}_{d} \boldsymbol{\Sigma}_{c} \boldsymbol{\Sigma}_{b} \boldsymbol{\Sigma}_{a}^{\frac{1}{2}}\right)^{2}\right)\right\}^{2} \\
& =\frac{15}{4} \frac{n_{b}^{2} n_{c}^{2} n_{d}^{2}}{p^{6} n_{a}}\left\{\frac{\operatorname{tr}\left(\left(\boldsymbol{\Sigma}_{a}^{\frac{1}{2}} \boldsymbol{\Sigma}_{b} \boldsymbol{\Sigma}_{c} \boldsymbol{\Sigma}_{d} \boldsymbol{\Sigma}_{a}^{\frac{1}{2}}+\boldsymbol{\Sigma}_{a}^{\frac{1}{2}} \boldsymbol{\Sigma}_{d} \boldsymbol{\Sigma}_{c} \boldsymbol{\Sigma}_{b} \boldsymbol{\Sigma}_{a}^{\frac{1}{2}}\right)^{2}\right)}{p}\right. \\
& \rightarrow 0 .
\end{aligned}
$$

- Proof of (5.6). For $h=n_{a}+1, \ldots, n_{a}+n_{b}$, it follows from Lemma 4.3 that

$$
\begin{aligned}
\mathrm{E}\left[D_{h}^{4}\right]= & \mathrm{E}\left[\left\{\frac{n_{c} n_{d}}{r_{p}} \operatorname{tr}\left(\boldsymbol{T}_{a}\left(n_{a}\right)\left(\boldsymbol{y}_{h-n_{a}} \boldsymbol{y}_{h-n_{a}}^{\prime}-\boldsymbol{\Sigma}_{b}\right) \boldsymbol{\Sigma}_{c} \boldsymbol{\Sigma}_{d}\right)\right\}^{4}\right] \\
= & \frac{n_{c}^{4} n_{d}^{4}}{r_{p}^{4}} \mathrm{E}\left[\left\{\operatorname{tr}\left(\boldsymbol{y}_{h-n_{a}}^{\prime} \boldsymbol{\Sigma}_{b}^{-\frac{1}{2}} \boldsymbol{\Sigma}_{b}^{\frac{1}{2}} \boldsymbol{\Sigma}_{c} \boldsymbol{\Sigma}_{d} \boldsymbol{T}_{a}\left(n_{a}\right) \boldsymbol{\Sigma}_{b}^{\frac{1}{2}} \boldsymbol{\Sigma}_{b}^{-\frac{1}{2}} \boldsymbol{y}_{h-n_{a}}\right)\right.\right. \\
& \left.\left.-\operatorname{tr}\left(\boldsymbol{\Sigma}_{b}^{\frac{1}{2}} \boldsymbol{\Sigma}_{c} \boldsymbol{\Sigma}_{d} \boldsymbol{T}_{a}\left(n_{a}\right) \boldsymbol{\Sigma}_{b}^{\frac{1}{2}}\right)\right\}^{4}\right] \\
= & \frac{n_{c}^{4} n_{d}^{4}}{r_{p}^{4}} \mathrm{E}\left[3 \operatorname{tr}\left(\left(\boldsymbol{B}+\boldsymbol{B}^{\prime}\right)^{4}\right)+\frac{3}{4}\left\{\operatorname{tr}\left(\left(\boldsymbol{B}+\boldsymbol{B}^{\prime}\right)^{2}\right)\right\}^{2}\right],
\end{aligned}
$$

where $\boldsymbol{B}=\boldsymbol{\Sigma}_{b}^{\frac{1}{2}} \boldsymbol{\Sigma}_{c} \boldsymbol{\Sigma}_{d} \boldsymbol{T}_{a}\left(n_{a}\right) \boldsymbol{\Sigma}_{b}^{\frac{1}{2}}$, so that

$$
\begin{aligned}
\mathrm{E}\left[D_{h}^{4}\right] & =\frac{n_{c}^{4} n_{d}^{4}}{r_{p}^{4}} \mathrm{E}\left[3 \operatorname{tr}\left(\left(\boldsymbol{B}+\boldsymbol{B}^{\prime}\right)^{4}\right)+\frac{3}{4}\left\{\operatorname{tr}\left(\left(\boldsymbol{B}+\boldsymbol{B}^{\prime}\right)^{2}\right)\right\}^{2}\right] \\
& <\frac{n_{c}^{4} n_{d}^{4}}{r_{p}^{4}} \mathrm{E}\left[3\left\{\operatorname{tr}\left(\left(\boldsymbol{B}+\boldsymbol{B}^{\prime}\right)^{2}\right)\right\}^{2}+\frac{3}{4}\left\{\operatorname{tr}\left(\left(\boldsymbol{B}+\boldsymbol{B}^{\prime}\right)^{2}\right)\right\}^{2}\right] \\
& =\frac{15}{4} \frac{n_{c}^{4} n_{d}^{4}}{r_{p}^{4}} \mathrm{E}\left[\left\{\operatorname{tr}\left(\left(\boldsymbol{B}+\boldsymbol{B}^{\prime}\right)^{2}\right)\right\}^{2}\right] \\
& =\frac{15}{4} \frac{n_{c}^{4} n_{d}^{4}}{r_{p}^{4}} \mathrm{E}\left[\left\{\operatorname{tr}\left(\left(\boldsymbol{\Sigma}_{b}^{\frac{1}{2}} \boldsymbol{\Sigma}_{c} \boldsymbol{\Sigma}_{d} \boldsymbol{T}_{a}\left(n_{a}\right) \boldsymbol{\Sigma}_{b}^{\frac{1}{2}}+\boldsymbol{\Sigma}_{b}^{\frac{1}{2}} \boldsymbol{T}_{a}\left(n_{a}\right) \boldsymbol{\Sigma}_{d} \boldsymbol{\Sigma}_{c} \boldsymbol{\Sigma}_{b}^{\frac{1}{2}}\right)^{2}\right)\right\}^{2}\right]
\end{aligned}
$$

Hence,

$$
\begin{aligned}
\sum_{h=n_{a}+1}^{n_{a}+n_{b}} \mathrm{E}\left[D_{h}^{4}\right] & \\
< & \frac{15}{4} \frac{n_{b} n_{c}^{4} n_{d}^{4}}{r_{p}^{4}} \mathrm{E}\left[\left\{\operatorname{tr}\left(\left(\boldsymbol{\Sigma}_{b}^{\frac{1}{2}} \boldsymbol{\Sigma}_{c} \boldsymbol{\Sigma}_{d} \boldsymbol{T}_{a}\left(n_{a}\right) \boldsymbol{\Sigma}_{b}^{\frac{1}{2}}+\boldsymbol{\Sigma}_{b}^{\frac{1}{2}} \boldsymbol{T}_{a}\left(n_{a}\right) \boldsymbol{\Sigma}_{d} \boldsymbol{\Sigma}_{c} \boldsymbol{\Sigma}_{b}^{\frac{1}{2}}\right)^{2}\right)\right\}^{2}\right] \\
= & \frac{15}{4} \frac{n_{c}^{2} n_{d}^{2}}{p^{8} n_{a}^{2} n_{b}} \\
& O\left(\mathrm{E}\left[\operatorname{tr}\left(\boldsymbol{T}_{a}\left(n_{a}\right) \boldsymbol{\Sigma}_{b} \boldsymbol{\Sigma}_{c} \boldsymbol{\Sigma}_{d} \boldsymbol{T}_{a}\left(n_{a}\right) \boldsymbol{\Sigma}_{b} \boldsymbol{\Sigma}_{c} \boldsymbol{\Sigma}_{d}\right) \operatorname{tr}\left(\boldsymbol{T}_{a}\left(n_{a}\right) \boldsymbol{\Sigma}_{b} \boldsymbol{\Sigma}_{c} \boldsymbol{\Sigma}_{d} \boldsymbol{T}_{a}\left(n_{a}\right) \boldsymbol{\Sigma}_{b} \boldsymbol{\Sigma}_{c} \boldsymbol{\Sigma}_{d}\right)\right]\right) \\
= & \frac{15}{4} \frac{n_{c}^{2} n_{d}^{2}}{p^{8} n_{a}^{2} n_{b}} O\left(n_{a}^{2} p^{4}\right) \\
\rightarrow & 0 .
\end{aligned}
$$


- Proof of (5.7). For $h=n_{a}+n_{b}+1, \ldots, n_{a}+n_{b}+n_{c}$, it follows from Lemma 4.3 that

$$
\begin{aligned}
\mathrm{E}\left[D_{h}^{4}\right]= & \mathrm{E}\left[\left\{\frac{n_{d}}{r_{p}} \operatorname{tr}\left(\boldsymbol{T}_{a}\left(n_{a}\right) \boldsymbol{T}_{b}\left(n_{b}\right)\left(\boldsymbol{z}_{h-n_{a}-n_{b}} \boldsymbol{z}_{h-n_{a}-n_{b}}^{\prime}-\boldsymbol{\Sigma}_{c}\right) \boldsymbol{\Sigma}_{d}\right)\right\}^{4}\right] \\
= & \frac{n_{d}^{4}}{r_{p}^{4}} \mathrm{E}\left[\left\{\boldsymbol{z}_{h-n_{a}-n_{b}} \boldsymbol{\Sigma}_{c}^{-\frac{1}{2}} \boldsymbol{\Sigma}_{c}^{\frac{1}{2}} \boldsymbol{\Sigma}_{d} \boldsymbol{T}_{a}\left(n_{a}\right) \boldsymbol{T}_{b}\left(n_{b}\right) \boldsymbol{\Sigma}_{c}^{\frac{1}{2}} \boldsymbol{\Sigma}_{c}^{-\frac{1}{2}} \boldsymbol{z}_{h-n_{a}-n_{b}}\right.\right. \\
& \left.\left.-\operatorname{tr}\left(\boldsymbol{\Sigma}_{c}^{\frac{1}{2}} \boldsymbol{\Sigma}_{d} \boldsymbol{T}_{a}\left(n_{a}\right) \boldsymbol{T}_{b}\left(n_{b}\right) \boldsymbol{\Sigma}_{c}^{\frac{1}{2}}\right)\right\}^{4}\right] \\
= & \frac{n_{d}^{4}}{r_{p}^{4}} \mathrm{E}\left[3 \operatorname{tr}\left(\left(\boldsymbol{C}+\boldsymbol{C}^{\prime}\right)^{4}\right)+\frac{3}{4}\left\{\operatorname{tr}\left(\left(\boldsymbol{C}+\boldsymbol{C}^{\prime}\right)^{2}\right)\right\}^{2}\right]
\end{aligned}
$$

where $\boldsymbol{C}=\boldsymbol{\Sigma}_{c}^{\frac{1}{2}} \boldsymbol{\Sigma}_{d} \boldsymbol{T}_{a}\left(n_{a}\right) \boldsymbol{T}_{b}\left(n_{b}\right) \boldsymbol{\Sigma}_{c}^{\frac{1}{2}}$, so that

$$
\begin{aligned}
\mathrm{E}\left[D_{h}^{4}\right] & =\frac{n_{d}^{4}}{r_{p}^{4}} \mathrm{E}\left[3 \operatorname{tr}\left(\left(\boldsymbol{C}+\boldsymbol{C}^{\prime}\right)^{4}\right)+\frac{3}{4}\left\{\operatorname{tr}\left(\left(\boldsymbol{C}+\boldsymbol{C}^{\prime}\right)^{2}\right)\right\}^{2}\right] \\
& <\frac{n_{d}^{4}}{r_{p}^{4}} \mathrm{E}\left[3\left\{\operatorname{tr}\left(\left(\boldsymbol{C}+\boldsymbol{C}^{\prime}\right)^{2}\right)\right\}^{2}+\frac{3}{4}\left\{\operatorname{tr}\left(\left(\boldsymbol{C}+\boldsymbol{C}^{\prime}\right)^{2}\right)\right\}^{2}\right] \\
& =\frac{15}{4} \frac{n_{d}^{4}}{r_{p}^{4}} \mathrm{E}\left[\left\{\operatorname{tr}\left(\left(\boldsymbol{C}+\boldsymbol{C}^{\prime}\right)^{2}\right)\right\}^{2}\right] \\
& =\frac{15}{4} \frac{n_{d}^{4}}{r_{p}^{4}} \mathrm{E}\left[\left\{\operatorname{tr}\left(\left(\boldsymbol{\Sigma}_{c}^{\frac{1}{2}} \boldsymbol{\Sigma}_{d} \boldsymbol{T}_{a}\left(n_{a}\right) \boldsymbol{T}_{b}\left(n_{b}\right) \boldsymbol{\Sigma}_{c}^{\frac{1}{2}}+\boldsymbol{\Sigma}_{c}^{\frac{1}{2}} \boldsymbol{T}_{b}\left(n_{b}\right) \boldsymbol{T}_{a}\left(n_{a}\right) \boldsymbol{\Sigma}_{d} \boldsymbol{\Sigma}_{c}^{\frac{1}{2}}\right)^{2}\right)\right\}^{2}\right]
\end{aligned}
$$

Hence, by using Proposition 4.8,

$$
\begin{aligned}
& \sum_{h=n_{a}+n_{b}+1}^{n_{a}+n_{b}+n_{c}} \mathrm{E}\left[D_{h}^{4}\right] \\
& <\frac{15}{4} \frac{n_{c} n_{d}^{4}}{r_{p}^{4}} \mathrm{E}\left[\left\{\operatorname{tr}\left(\left(\boldsymbol{\Sigma}_{c}^{\frac{1}{2}} \boldsymbol{\Sigma}_{d} \boldsymbol{T}_{a}\left(n_{a}\right) \boldsymbol{T}_{b}\left(n_{b}\right) \boldsymbol{\Sigma}_{c}^{\frac{1}{2}}+\boldsymbol{\Sigma}_{c}^{\frac{1}{2}} \boldsymbol{T}_{b}\left(n_{b}\right) \boldsymbol{T}_{a}\left(n_{a}\right) \boldsymbol{\Sigma}_{d} \boldsymbol{\Sigma}_{c}^{\frac{1}{2}}\right)^{2}\right)\right\}^{2}\right] \\
& \sim \frac{15}{4} \frac{n_{c} n_{d}^{4}}{r_{p}^{4}} \mathrm{E}\left[\left\{\operatorname{tr}\left(\boldsymbol{T}_{a}\left(n_{a}\right) \boldsymbol{\Sigma}_{d} \boldsymbol{\Sigma}_{c} \boldsymbol{\Sigma}_{d} \boldsymbol{T}_{a}\left(n_{a}\right) \boldsymbol{T}_{b}\left(n_{b}\right) \boldsymbol{\Sigma}_{c} \boldsymbol{T}_{b}\left(n_{b}\right)\right)\right\}^{2}\right] \\
& =\frac{15}{4} \frac{n_{c} n_{d}^{4}}{r_{p}^{4}} \mathrm{E}\left[\operatorname{tr}\left(\boldsymbol{T}_{a}\left(n_{a}\right) \boldsymbol{\Sigma}_{d} \boldsymbol{\Sigma}_{c} \boldsymbol{\Sigma}_{d} \boldsymbol{T}_{a}\left(n_{a}\right) \boldsymbol{T}_{b}\left(n_{b}\right) \boldsymbol{\Sigma}_{c} \boldsymbol{T}_{b}\left(n_{b}\right)\right)\right. \\
& \left.\quad \times \operatorname{tr}\left(\boldsymbol{T}_{a}\left(n_{a}\right) \boldsymbol{\Sigma}_{d} \boldsymbol{\Sigma}_{c} \boldsymbol{\Sigma}_{d} \boldsymbol{T}_{a}\left(n_{a}\right) \boldsymbol{T}_{b}\left(n_{b}\right) \boldsymbol{\Sigma}_{c} \boldsymbol{T}_{b}\left(n_{b}\right)\right)\right] \\
& =\frac{15}{4} \frac{n_{d}^{2}}{p^{8} n_{a}^{2} n_{b}^{2} n_{c}} O\left(n_{a}^{2} n_{b}^{2} p^{6}\right) \\
& \rightarrow 0 .
\end{aligned}
$$

- Proof of (5.8). For $h=n_{a}+n_{b}+n_{c}+1, \ldots, n_{a}+n_{b}+n_{c}+n_{d}$, it follows from Lemma 4.3 that

$$
\begin{aligned}
\mathrm{E}\left[D_{h}^{4}\right]= & \mathrm{E}\left[\left\{\frac{1}{r_{p}} \operatorname{tr}\left(\boldsymbol{T}_{a}\left(n_{a}\right) \boldsymbol{T}_{b}\left(n_{b}\right) \boldsymbol{T}_{c}\left(n_{c}\right)\left(\boldsymbol{w}_{h-n_{a}-n_{b}-n_{c}} \boldsymbol{w}_{h-n_{a}-n_{b}-n_{c}}^{\prime}-\boldsymbol{\Sigma}_{d}\right)\right)\right\}^{4}\right] \\
= & \frac{1}{r_{p}^{4}} \mathrm{E}\left[\left\{\boldsymbol{w}_{h-n_{a}-n_{b}-n_{c}}^{\prime} \boldsymbol{\Sigma}_{d}^{-\frac{1}{2}} \boldsymbol{\Sigma}_{d}^{\frac{1}{2}} \boldsymbol{T}_{a}\left(n_{a}\right) \boldsymbol{T}_{b}\left(n_{b}\right) \boldsymbol{T}_{c}\left(n_{c}\right) \boldsymbol{\Sigma}_{d}^{\frac{1}{2}} \boldsymbol{\Sigma}_{d}^{-\frac{1}{2}} \boldsymbol{w}_{h-n_{a}-n_{b}-n_{c}}\right.\right. \\
& \left.\left.-\operatorname{tr}\left(\boldsymbol{\Sigma}_{d}^{\frac{1}{2}} \boldsymbol{T}_{a}\left(n_{a}\right) \boldsymbol{T}_{b}\left(n_{b}\right) \boldsymbol{T}_{c}\left(n_{c}\right) \boldsymbol{\Sigma}_{d}^{\frac{1}{2}}\right)\right\}^{4}\right] \\
= & \frac{1}{r_{p}^{4}} \mathrm{E}\left[3 \operatorname{tr}\left(\left(\boldsymbol{D}+\boldsymbol{D}^{\prime}\right)^{4}\right)+\frac{3}{4}\left\{\operatorname{tr}\left(\left(\boldsymbol{D}+\boldsymbol{D}^{\prime}\right)^{2}\right)\right\}^{2}\right],
\end{aligned}
$$

where $\boldsymbol{D}=\boldsymbol{\Sigma}_{d}^{\frac{1}{2}} \boldsymbol{T}_{a}\left(n_{a}\right) \boldsymbol{T}_{b}\left(n_{b}\right) \boldsymbol{T}_{c}\left(n_{c}\right) \boldsymbol{\Sigma}_{d}^{\frac{1}{2}}$, so that

$$
\mathrm{E}\left[D_{h}^{4}\right]=\frac{1}{r_{p}^{4}} \mathrm{E}\left[3 \operatorname{tr}\left(\left(\boldsymbol{D}+\boldsymbol{D}^{\prime}\right)^{4}\right)+\frac{3}{4}\left\{\operatorname{tr}\left(\left(\boldsymbol{D}+\boldsymbol{D}^{\prime}\right)^{2}\right)\right\}^{2}\right]
$$




$$
\begin{aligned}
& <\frac{1}{r_{p}^{4}} \mathrm{E}\left[3\left(\operatorname{tr}\left(\left(\boldsymbol{D}+\boldsymbol{D}^{\prime}\right)^{2}\right)\right)^{2}+\frac{3}{4}\left\{\operatorname{tr}\left(\left(\boldsymbol{D}+\boldsymbol{D}^{\prime}\right)^{2}\right)\right\}^{2}\right] \\
& =\frac{15}{4} \frac{1}{r_{p}^{4}} \mathrm{E}\left[\left\{\operatorname{tr}\left(\left(\boldsymbol{D}+\boldsymbol{D}^{\prime}\right)^{2}\right)\right\}^{2}\right] \\
& =\frac{15}{4} \frac{1}{r_{p}^{4}} \mathrm{E}\left[\left\{\operatorname{tr}\left(\left(\boldsymbol{\Sigma}_{d}^{\frac{1}{2}} \boldsymbol{T}_{a}\left(n_{a}\right) \boldsymbol{T}_{b}\left(n_{b}\right) \boldsymbol{T}_{c}\left(n_{c}\right) \boldsymbol{\Sigma}_{d}^{\frac{1}{2}}+\boldsymbol{\Sigma}_{d}^{\frac{1}{2}} \boldsymbol{T}_{c}\left(n_{c}\right) \boldsymbol{T}_{b}\left(n_{b}\right) \boldsymbol{T}_{a}\left(n_{a}\right) \boldsymbol{\Sigma}_{d}^{\frac{1}{2}}\right)\right)\right\}^{2}\right] .
\end{aligned}
$$

Hence,

$$
\begin{aligned}
& \sum_{h=n_{a}+n_{b}+n_{c}+1}^{n_{a}+n_{b}+n_{c}+n_{d}} \mathrm{E}\left[D_{h}^{4}\right] \\
< & \frac{15}{4} \frac{n_{d}}{r_{p}^{4}} \mathrm{E}\left[\left\{\operatorname{tr}\left(\left(\boldsymbol{\Sigma}_{d}^{\frac{1}{2}} \boldsymbol{T}_{a}\left(n_{a}\right) \boldsymbol{T}_{b}\left(n_{b}\right) \boldsymbol{T}_{c}\left(n_{c}\right) \boldsymbol{\Sigma}_{d}^{\frac{1}{2}}+\boldsymbol{\Sigma}_{d}^{\frac{1}{2}} \boldsymbol{T}_{c}\left(n_{c}\right) \boldsymbol{T}_{b}\left(n_{b}\right) \boldsymbol{T}_{a}\left(n_{a}\right) \boldsymbol{\Sigma}_{d}^{\frac{1}{2}}\right)^{2}\right)\right\}^{2}\right] \\
\sim & \frac{15}{4} \frac{n_{d}}{r_{p}^{4}} \mathrm{E}\left[\left\{\operatorname{tr}\left(\boldsymbol{T}_{a}\left(n_{a}\right) \boldsymbol{\Sigma}_{d} \boldsymbol{T}_{a}\left(n_{a}\right) \boldsymbol{T}_{b}\left(n_{b}\right) \boldsymbol{T}_{c}\left(n_{c}\right) \boldsymbol{\Sigma}_{d} \boldsymbol{T}_{c}\left(n_{c}\right) \boldsymbol{T}_{b}\left(n_{b}\right)\right)\right\}^{2}\right] \\
= & \frac{15}{4} \frac{n_{d}}{r_{p}^{4}} \mathrm{E}\left[\operatorname{tr}\left(\boldsymbol{T}_{a}\left(n_{a}\right) \boldsymbol{\Sigma}_{d} \boldsymbol{T}_{a}\left(n_{a}\right) \boldsymbol{T}_{b}\left(n_{b}\right) \boldsymbol{T}_{c}\left(n_{c}\right) \boldsymbol{\Sigma}_{d} \boldsymbol{T}_{c}\left(n_{c}\right) \boldsymbol{T}_{b}\left(n_{b}\right)\right)\right. \\
& \left.\times \operatorname{tr}\left(\boldsymbol{T}_{a}\left(n_{a}\right) \boldsymbol{\Sigma}_{d} \boldsymbol{T}_{a}\left(n_{a}\right) \boldsymbol{T}_{b}\left(n_{b}\right) \boldsymbol{T}_{c}\left(n_{c}\right) \boldsymbol{\Sigma}_{d} \boldsymbol{T}_{c}\left(n_{c}\right) \boldsymbol{T}_{b}\left(n_{b}\right)\right)\right] \\
= & \frac{15}{4} \frac{1}{p^{8} n_{a}^{2} n_{b}^{2} n_{c}^{2} n_{d}}\left\{O\left(n_{a}^{4} n_{b}^{4} n_{c}^{4} p^{2}\right)+O\left(n_{a}^{2} n_{b}^{4} n_{c}^{4} p^{4}\right)+O\left(n_{a}^{4} n_{b}^{2} n_{c}^{4} p^{4}\right)+O\left(n_{a}^{4} n_{b}^{4} n_{c}^{2} p^{4}\right)\right. \\
& \left.+O\left(n_{a}^{2} n_{b}^{2} n_{c}^{4} p^{6}\right)+O\left(n_{a}^{2} n_{b}^{4} n_{c}^{2} p^{6}\right)+O\left(n_{a}^{4} n_{b}^{2} n_{c}^{2} p^{6}\right)+O\left(n_{a}^{2} n_{b}^{2} n_{c}^{2} p^{8}\right)\right\} \\
= & \frac{15}{4} \frac{1}{p^{8} n_{a}^{2} n_{b}^{2} n_{c}^{2} n_{d}} O\left(n_{a}^{2} n_{b}^{2} n_{c}^{2} p^{8}\right) \\
\rightarrow & 0 .
\end{aligned}
$$

This completes the proof of Lemma 2.4.

\section{Acknowledgments}

The first author was supported in part by Japan Society for the Promotion of Science KAKENHI Grant Number $18 K 13454$. This study was partly carried out when the first author (KT) was a member of Graduate School of Arts and Sciences, the University of Tokyo.

\section{References}

[1] Bai, Z.; Saranadasa, H. (1996). Effect of high dimension: by an example of a two sample problem. Statist. Sinica 6 , no.2, 311-329.

[2] Boente, G.; Pires, A. M.; Rodrigues, I. M. (2009). Robust tests for the common principal components model. $J$. Statist. Plann. Inference 139, no.4, 1332-1347.

[3] Boik, R.J. (2002). Spectral models for covariance matrices. Biometrika 89, no.1, 159-182.

[4] Chen, S.X.; Zhang, L.-X.; Zhong, P.-S. (2010). Tests for high-dimensional covariance matrices. J. Amer. Statist. Assoc. 105, no.490, 810-819.

[5] Flury, B.N. (1984). Common principal components in $k$ groups. J. Amer. Statist. Assoc. 79, no.388, 892-898.

[6] Flury, B.N. (1986). Asymptotic theory for common principal component analysis. Ann. Statist. 14, no.2, 418-430.

[7] Flury, B. (1988). Common principal components and related multivariate models. Wiley Series in Probability and Mathematical Statistics: Applied Probability and Statistics. John Wiley \& Sons, Inc., New York.

[8] Hallin, M.; Paindaveine, D.; Verdebout, T. (2008). Pseudo-Gaussian inference in heterokurtic elliptical common principal components models. Ann. I.S.U.P. 52, no.1-2, 9-24. 
[9] Hallin, M.; Paindaveine, D.; Verdebout, T. (2010). Testing for common principal components under heterokurticity. J. Nonparametr. Stat. 22, no.7, 879-895.

[10] Hallin, M.; Paindaveine, D.; Verdebout, T. (2013). Optimal rank-based tests for common principal components. Bernoulli 19, no.5B, 2524-2556.

[11] Li, J.; Chen, S.X. (2012). Two sample tests for high-dimensional covariance matrices. Ann. Statist. 40, no. 2, 908-940.

[12] Liu, B.; Xu, L.; Zheng, S.; Tian, G.-L. (2014). A new test for the proportionality of two large-dimensional covariance matrices. J. Multivariate Anal. 131, 293-308.

[13] Schott, J.R.(2007) A test for the equality of covariance matrices when the dimension is large relative to the sample sizes. Comput. Statist. Data Anal. 51, no.12, 6535-6542.

[14] Srivastava, M.S. (2005). Some tests concerning the covariance matrix in high dimensional data. J. Japan Statist. Soc. 35, no.2, 251-272.

[15] Srivastava, M.S.; Yanagihara, H. (2010). Testing the equality of several covariance matrices with fewer observations than the dimension. J. Multivariate Anal. 101, no.6, 1319-1329.

[16] Srivastava, M.S.; Yanagihara, H.; Kubokawa, T. (2014). Tests for covariance matrices in high dimension with less sample size. J. Multivariate Anal. 130, 289-309.

[17] Tsukuda, K.; Matsuura, S. (2019). High-dimensional testing for proportional covariance matrices. J. Multivariate Anal. 171, 412-420.

[18] Xu, L.; Liu, B.; Zheng, S.; Bao, S. (2014). Testing proportionality of two large-dimensional covariance matrices. Comput. Statist. Data Anal. 78, 43-55. 